\title{
BREAKING THE COHERENCE BARRIER: A NEW THEORY FOR COMPRESSED SENSING
}

\author{
BEN ADCOCK ${ }^{1}$, ANDERS C. HANSEN ${ }^{2,3}$, \\ CLARICE POON $^{2}$ and BOGDAN ROMAN ${ }^{2}$ \\ ${ }^{1}$ Department of Mathematics, Simon Fraser University, Burnaby BC, V5A 1S6, Canada; \\ email: ben_adcock@sfu.ca \\ ${ }^{2}$ DAMTP, University of Cambridge, Cambridge CB3 0WA, UK; \\ email: ach70@cam.ac.uk,cmhsp2@cam.ac.uk, abr28@cam.ac.uk \\ ${ }^{3}$ Department of Mathematics, University of Oslo, 0316 OSLO, Norway
}

Received 29 October 2014; accepted 1 November 2016

\begin{abstract}
This paper presents a framework for compressed sensing that bridges a gap between existing theory and the current use of compressed sensing in many real-world applications. In doing so, it also introduces a new sampling method that yields substantially improved recovery over existing techniques. In many applications of compressed sensing, including medical imaging, the standard principles of incoherence and sparsity are lacking. Whilst compressed sensing is often used successfully in such applications, it is done largely without mathematical explanation. The framework introduced in this paper provides such a justification. It does so by replacing these standard principles with three more general concepts: asymptotic sparsity, asymptotic incoherence and multilevel random subsampling. Moreover, not only does this work provide such a theoretical justification, it explains several key phenomena witnessed in practice. In particular, and unlike the standard theory, this work demonstrates the dependence of optimal sampling strategies on both the incoherence structure of the sampling operator and on the structure of the signal to be recovered. Another key consequence of this framework is the introduction of a new structured sampling method that exploits these phenomena to achieve significant improvements over current state-of-the-art techniques.
\end{abstract}

2010 Mathematics Subject Classification: 94A20, 94A08 (primary); 42C40, 65R32, 92C55 (secondary) 


\section{Introduction}

Introduced formally around a decade ago, compressed sensing (CS) [18, 32] has since become a popular area of research in mathematics, computer science and engineering [14, 23, 29, 35, 36, 39-41]. In many real-world problems one is limited by the amount of data that can be collected, making reconstruction via classical techniques impossible. The theory and techniques of CS provide a means to reconstruct from fewer measurements, giving it potential to significantly enhance the recovery step in such applications.

The theory of CS is based on three key concepts: sparsity, incoherence and random sampling. Whilst there are applications where these apply, in many practical problems one or more of these principles may be lacking. This includes most applications in medical imaging-Magnetic Resonance Imaging (MRI), Computerized Tomography (CT) and other versions of tomography such as Thermoacoustic, Photoacoustic or Electrical Impedance Tomography-electron microscopy, as well as seismic tomography, fluorescence microscopy, Hadamard spectroscopy, Helium Atom Scattering (HAS) and radio interferometry. In many of these problems, it is the principle of incoherence that is lacking, rendering the standard theory inapplicable. Yet, despite this issue CS has been, and continues to be, used successfully in many of these areas. To do so, however, it is typically implemented with sampling strategies that differ substantially from the uniform random sampling strategies suggested by the theory. In fact, in many cases the sampling strategies suggested by existing theory yield highly suboptimal numerical results.

The mathematical theory of CS has now reached a mature state. However, as this discussion suggests, there is a substantial, and arguably widening gap between theory and its applications. New developments and sampling strategies are increasingly based on empirical evidence lacking mathematical justification. Furthermore, in the above applications one also witnesses a number of intriguing phenomena that are not explained by the standard theory. For example, in such problems, the optimal sampling strategy depends not just on the overall sparsity of the signal, but also on its structure; a fact that will be documented thoroughly in this paper. This phenomenon is in contradiction with the usual sparsity-based theory of CS. Theorems that explain this observation-that is that reflect how the optimal subsampling strategy depends on the structure of the signal—do not currently exist.

1.1. Contributions. The purpose of this paper is to provide a bridge across this divide. It does so by introducing a theoretical framework for CS based on three more general principles: asymptotic sparsity, asymptotic incoherence 
and multilevel random sampling. This new framework shows that CS is also possible under these substantially more general conditions, and moreover, can convey some key advantages. Importantly, it also addresses the issue raised above: namely, the dependence of the subsampling strategy on the structure of the signal.

The significance of this generalization is threefold. First, as will be explained, inverse problems arising from the aforementioned applications are often not incoherent and sparse, but asymptotically incoherent and asymptotically sparse. This paper provides the first comprehensive mathematical explanation for a range of empirical usages of CS in applications such as those listed above. Second, in showing that incoherence is not a requirement for CS, but instead that asymptotic incoherence suffices, the new theory offers markedly greater flexibility in the design of sensing mechanisms. In the future, sensors need only satisfy this significantly more relaxed condition. Third, by using asymptotic incoherence and multilevel sampling to exploit not just sparsity, but also structure, that is asymptotic sparsity, this frameworks paves the way for improved sensing paradigms in CS that achieve better reconstructions in practice than current stateof-the-art CS techniques.

A key aspect of many practical problems, including those listed above, is that they do not offer the freedom to design or choose the sensing operator, but instead impose it (for example, Fourier sampling in MRI). As such, much existing CS work, which relies on random or custom-designed sensing matrices (typically to provide universality), is not applicable. This paper shows that in many such applications the imposed sensing operators are both nonuniversal and highly coherent with popular sparsifying bases. Yet they are often asymptotically incoherent, and thus fall within the remit of the new framework. Spurred by this observation, this paper also raises the question of whether universality and incoherence are actually desirable in practice, even in applications where there is flexibility to design sensing operators with this property (for example, in compressive imaging). Our theorems show that asymptotically incoherent sensing and multilevel sampling allow one to exploit asymptotic, as opposed to just global sparsity. Doing so leads to notable advantages over incoherent sensing, even for problems where the latter are applicable. Moreover, and crucially, this can be done in a computationally efficient manner using fast Fourier or Hadamard transforms (see Section 6.1).

Our framework applies to any CS scenario where both the coherence and sparsity are nonuniform. Of the many applications where this is the case, one of the most important corresponds to the problem of Fourier sampling with multiresolution sparsifying transforms such as wavelets. This model arises in applications such as MRI, CT, radio interferometry, HAS and elsewhere. When applied to this specific problem, this framework yields new and near-optimal 
sampling strategies based on multilevel random subsampling, and a new series of recovery guarantees. Specifically, a corollary of our abstract results in the case of Fourier sampling with wavelets taking the following form. If $s_{k}$ denotes the sparsity of the wavelet coefficients of a given signal or image in the $k$ th wavelet scale, then to recover those coefficients one requires

$$
m_{k} \gtrsim\left(s_{k}+\sum_{\substack{l=1 \\ l \neq k}}^{r} \beta^{-|k-l|} s_{l}\right), \quad k=1, \ldots, r .
$$

Fourier measurements (up to log factors) taken uniformly at random from the corresponding $k$ th sampling level (a dyadic band in frequency space), where $\beta>1$ is a constant depending on the type of wavelet used. See Section 6 for further discussion. As we explain, this guarantee not only confirms the empirically observed recovery properties of CS for such problems, but it also explains some of the key phenomena witnessed; for example, the dependence of the optimal sampling strategy on the sparsity structure.

Another contribution of this paper is that the theorems proved apply in both the finite- and infinite-dimensional settings. Many of the problems listed above are analogue, that is modelled with continuous operators such as the Fourier or Radon transforms. Conversely, the standard CS is based on a finite-dimensional model. Such a mismatch between the computational and the physical model can lead to critical errors when CS techniques are applied to real data arising from continuous models, or inverse crimes when the data is inappropriately simulated $[22,46]$. To overcome this issue, a theory of CS in infinite dimensions was recently introduced in [3]. This paper extends [3] by presenting the new framework in both the finiteand infinite-dimensional settings. We note in passing that the infinite-dimensional analysis is instrumental for obtaining the Fourier sampling with wavelet sparsity estimate (1.1).

This aside, an additional outcome of this work is that the Restricted Isometry Property (RIP), although a popular tool in CS theory, is of little relevance in many practical inverse problems. As confirmed later via the so-called flip test, the standard RIP cannot hold in these types of applications.

The fact that the RIP may be too strong an assumption in practice is well known in the standard CS literature. To overcome this, incoherence-based results, which avoid the RIP, have shown that CS is indeed possible under weaker conditions (see $[16,17]$ and references therein). We remark that the reasons for the absence of the RIP in the standard CS setting are fundamentally different and arguably less significant to the reasons for its absence in this setting. In particular, as we demonstrate, in many applications only very specific structured sparse vectors can be recovered. This is in stark contrast to the standard understanding that all sparse 
vectors can be recovered equally well regardless of their structure. We refer to Section 2.3 for details.

Finally, we remark that this is primarily a mathematical paper. However, as one may expect in light of the above discussion, there are a range of practical implications. We therefore encourage the reader to consult [73] for further elaboration on the practical aspects and more extensive numerical experiments. We also remark that the practical importance of the new concepts of asymptotic sparsity, asymptotic incoherence and multilevel random subsampling has already been verified experimentally in a realistic MRI setting by Siemens [84] (based on an earlier preprint of this paper). Siemens' conclusion from their experiments is: '[...] The image resolution has been greatly improved [...]. Current results practically demonstrated that it is possible to break the coherence barrier by increasing the spatial resolution in MR acquisitions. This likewise implies that the full potential of the CS is unleashed only if asymptotic sparsity and asymptotic incoherence is achieved. Therefore, CS might better be used to increase the spatial resolution rather than accelerating the data acquisition in the context of nondynamic 3D MR imaging.'

1.2. Relation to other works. Since the early days of CS, there have been numerous investigations into settings which go beyond classical sparsity and incoherence. So-called structured sparsity has been studied extensively, and there are now a range of generalized sparsity notions in the literature. (Structured sparsity, especially multiscale-type sparsity, also predates CS by some yearssee, for example, the work of Donoho and Huo [33] — and finds use outside of CS—see, for example, the work of Donoho and Kutyniok on geometric separation [34].) These include group, block, weighted and tree sparsity, amongst others (see $[8,11,38,72,80]$ and references therein). In most of these works, structured sparsity is exploited by the design of the recovery algorithm (for example, by replacing the thresholding step in an iterative algorithm or the regularization functional in an optimization approach), with the sensing being carried out by a standard, incoherent operator (for example, a Gaussian random matrix). Our framework differs from these works in that it applies directly to the practical, and asymptotically incoherent, sensing operators imposed by many applications and to the way in which CS is typically implemented in practice in these applications. It demonstrates why good recovery is possible in these practical settings via the notion of asymptotic sparsity, and lends crucial insight into the design of optimal sampling strategies.

The observation that many applications of CS result in nonuniform coherence patterns arguably dates back to Lustig et al.'s seminal work in CS for MRI [58, 6163]. For Fourier sampling, numerous empirical sampling strategies have been 
proposed to overcome this problem $[61,85]$, and several other works have followed more principled approaches based on designing sampling strategies to match the underlying coherence pattern (see $[10,55,70,71]$ and references therein). However, these works do not explain the key role played by asymptotic sparsity in the CS recovery. Our work does this, and provides sampling strategies which are provably optimal with respect to both the sparsity and the coherence structures.

As mentioned, an important instance of our framework is that case of wavelet sparsifying transforms. The idea of sampling the low-order wavelet coefficients of an image differently goes back to the early days of CS. In particular, Donoho considers a two-level approach for recovering wavelet coefficients in his seminal paper [32], based on acquiring the coarse scales coefficients directly. This was later extended by Tsaig and Donoho to so-called 'multiscale CS' in [82], where distinct subbands were sensed separately. See also [17, 74]. Unlike in our framework, these works normally assume a separation of the wavelet coefficients into distinct bands before sampling, which is largely infeasible in practice (in particular, any application based on Fourier or Hadamard sensing). We note also that similar sampling strategies to those that we introduce here are found in most implementations of CS in MRI [62, 63, 70, 71]. In addition, a so-called 'half-half' scheme (an example of a two-level strategy in our terminology-see Section 3) was used by [77] in an application of CS in fluorescence microscopy, albeit without theoretical recovery guarantees.

The proofs of the main results in this paper have their roots in some existing ideas from CS literature [3, 16, 43], with the two key tools being dual certificates and the golfing scheme. However, in order to account for the sparsity structure and the different sampling patterns used the techniques have some significant differences. In addition, as pointed out in [44, page 26], the original proofs using the golfing scheme assume an independence of certain random variables that will never be satisfied in general. The techniques used in this paper are different and overcome this issue yielding complete generality. Moreover, unlike almost all existing works, our results address both the finite- and infinite-dimensional CS settings. This extends (and improves) a line of work initiated in [3], and calls for a number of more sophisticated mathematical techniques.

REMARK 1.1. Since the initial preprint of this work, there have been several other applications and extensions inspired by the first version which we now mention for the reader's benefit. First, the multilevel sampling strategies we introduced have been extended to block sampling strategies [10, 12, 20, 21], which are more practical for applications such as MRI. A type of multilevel subsampling has also be considered in $[49,50]$ in the context of practical 
compressive imaging architectures, with application to single-pixel [37, 78] and lensless imaging [86]. Our main results in this paper provide a theoretical foundation for these implementations. There have also been several theoretical extensions. First, generalizations of the RIP for the setting of asymptotic sparsity, asymptotic incoherence and multilevel random subsampling have been introduced and analysed in $[9,60,80]$. These complement the results proved in this paper by establishing uniform recovery guarantees. Second, there have been extensions to redundant sparsifying transforms [69] (see also [57] for the case of shearlets) and to total variation minimization [68], respectively.

\section{Motivation}

In this section, we discuss how the standard theory of CS falls short in explaining its empirical success in many applications. Specifically, even in wellknown applications such as MRI (note that MRI was one of the first applications of CS [58, 61-63]), there is a significant gap between theory and practice.

2.1. Compressed sensing. We commence with a short review of aspects of finite-dimensional CS theory (infinite-dimensional CS will be considered in Section 5). Since CS has been the subject of a body of research in the last decade we will not attempt a full survey here, opting instead to focus on aspects most relevant to this paper. For much more comprehensive reviews, including historical context and discussion, we refer to [14, 29, 35, 36, 39-41].

A typical setup in CS, and one which we shall follow in part of this paper, is as follows. Let $\left\{\psi_{j}\right\}_{j=1}^{N}$ and $\left\{\varphi_{j}\right\}_{j=1}^{N}$ be two orthonormal bases of $\mathbb{C}^{N}$, the sampling and sparsity bases respectively, and write $U=\left(u_{i j}\right)_{i, j=1}^{N} \in \mathbb{C}^{N \times N}, u_{i j}=\left\langle\varphi_{j}, \psi_{i}\right\rangle$. Note that $U$ is an isometry, that is $U^{*} U=I$.

Definition 2.1. Let $U=\left(u_{i j}\right)_{i, j=1}^{N} \in \mathbb{C}^{N \times N}$ be an isometry. The coherence of $U$ is precisely

$$
\mu(U)=\max _{i, j=1, \ldots, N}\left|u_{i j}\right|^{2} \in\left[N^{-1}, 1\right] .
$$

We say that $U$ is perfectly incoherent if $\mu(U)=N^{-1}$.

A signal $f \in \mathbb{C}^{N}$ is said to be $s$-sparse in the orthonormal basis $\left\{\varphi_{j}\right\}_{j=1}^{N}$ if at most $s$ of its coefficients in this basis are nonzero. In other words, $f=\sum_{j=1}^{N} x_{j} \varphi_{j}$, and the vector $x \in \mathbb{C}^{N}$ satisfies $|\operatorname{supp}(x)| \leqslant s$, where $\operatorname{supp}(x)=\left\{j: x_{j} \neq 0\right\}$. Let $f \in \mathbb{C}^{N}$ be $s$-sparse in $\left\{\varphi_{j}\right\}_{j=1}^{N}$, and suppose we have access to the samples $\hat{f}_{j}=\left\langle f, \psi_{j}\right\rangle, j=1, \ldots, N$. Let $\Omega \subseteq\{1, \ldots, N\}$ be of cardinality $m$ and chosen 
uniformly at random. According to a result of Candès and Plan [16] and Adcock and Hansen [3], $f$ can be recovered exactly with probability exceeding $1-\epsilon$ from the subset of measurements $\left\{\hat{f}_{j}: j \in \Omega\right\}$, provided

$$
m \gtrsim \mu(U) \cdot N \cdot s \cdot\left(1+\log \left(\epsilon^{-1}\right)\right) \cdot \log (N),
$$

(here and elsewhere in this paper we shall use the notation $a \gtrsim b$ to mean that there exists a constant $C>0$ independent of all relevant parameters such that $a \geqslant C b$ ). In practice, recovery can be achieved by solving the following convex optimization problem:

$$
\min _{\eta \in \mathbb{C}^{N}}\|\eta\|_{l^{1}} \text { subject to } P_{\Omega} U \eta=P_{\Omega} \hat{f}
$$

where $\hat{f}=\left(\hat{f}_{1}, \ldots, \hat{f}_{N}\right)^{\top}$ and $P_{\Omega} \in \mathbb{C}^{N \times N}$ is the diagonal projection matrix with $j$ th entry 1 if $j \in \Omega$ and zero otherwise. The key estimate (2.2) shows that the number of measurements $m$ required is, up to a log factor, on the order of the sparsity $s$, provided the coherence $\mu(U)=\mathcal{O}\left(N^{-1}\right)$. This is the case, for example, when $U$ is the DFT matrix; a problem which was studied in some of the first papers on CS [18].

2.2. Incoherence is often lacking. As mentioned, in a number of important applications, not least MRI, the sampling is carried out in the Fourier domain. Since images are approximately sparse in wavelets, the usual CS setup is to form the matrix $U_{N}=U_{\mathrm{df}} V_{\mathrm{dw}}^{-1} \in \mathbb{C}^{N \times N}$, where $U_{\mathrm{df}}$ and $V_{\mathrm{dw}}$ represent the discrete Fourier and wavelet transforms, respectively.

Unfortunately, in the case the coherence satisfies $\mu\left(U_{N}\right)=\mathcal{O}(1)$ as $N \rightarrow \infty$, for any wavelet basis. Thus, this problem has the worst possible coherence, and the standard CS estimate (2.2) states that $m=N$ samples are needed in this case (that is full sampling), even though the object to recover is typically highly sparse. Note that this is not an insufficiency of the theory: as seen in Figure 1, uniform random subsampling in this problem yields an extremely poor reconstruction.

Although the presence of high coherence has been well documented in the MRI context [61-63], the source of it has not been fully explained. As it transpires, the underlying reason for this lack of incoherence can be traced to the fact that this finite-dimensional problem is a discretization of an infinite-dimensional problem. Specifically,

$$
\underset{N \rightarrow \infty}{\text { WOT-lim }} U_{\mathrm{df}} V_{\mathrm{dw}}^{-1}=U,
$$

where $U: l^{2}(\mathbb{N}) \rightarrow l^{2}(\mathbb{N})$ is the operator represented as the infinite matrix

$$
U=\left\{\left\langle\varphi_{i}, \psi_{j}\right\rangle\right\}_{i, j \in \mathbb{N}},
$$



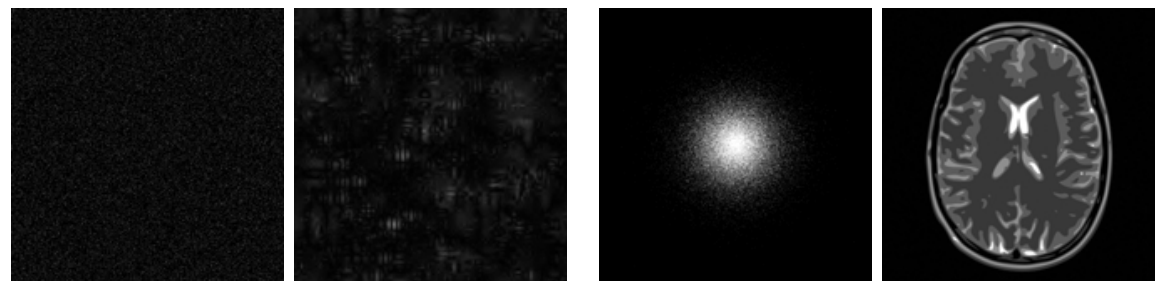

Figure 1. Left to right: (i) 5\% uniform random subsampling scheme, (ii) CS reconstruction from uniform subsampling, (iii) 5\% multilevel subsampling scheme, (iv) CS reconstruction from multilevel subsampling.

and the functions $\varphi_{j}$ are the wavelets used, the $\psi_{j}$ 's are the standard complex exponentials and WOT denotes the weak operator topology. Since the coherence of the infinite matrix $U$-that is the supremum of its entries in absolute value-is a fixed number independent of the discretization $N$, we cannot expect incoherence of the discretization $U_{N}$ (that is, $U_{N}=\mathcal{O}\left(N^{-1}\right)$ ) for large $N$. In other words, at some point one will always encounter the so-called coherence barrier. To mitigate this problem, one may naturally try to change $\left\{\varphi_{j}\right\}$ or $\left\{\psi_{j}\right\}$. However, this will deliver only marginal benefits: (2.4) demonstrates that the coherence barrier will always occur for large enough $N$, regardless of the choice of the bases.

Note that this issue is not isolated to this particular example. Informally, any problem that arises as a discretization of an infinite-dimensional problem will suffer from the same phenomenon. The list of applications of this type is long, and includes, for example, MRI, CT, microscopy and seismology.

In view of the coherence barrier, one may wonder how it is possible that CS is applied so successfully to many such problems. The key is so-called asymptotic incoherence (see Section 3.1) and the use of variable density subsampling strategies. The success of such a subsampling is well known in the CS MRI community $[61-63,70,71]$ and is confirmed numerically in Figure 1 . However, it is important to note that this is an empirical solution to the problem. None of the usual CS theory discussed in Section 2.1 explains the effectiveness of CS when implemented in this way.

2.3. Sparsity, the flip test and the absence of RIP. The previous discussion demonstrates that we must dispense with the principles of incoherence and uniform random subsampling in order to develop a new framework. We now claim that sparsity too must also be replaced with a more general concept. This may come as a surprise to the reader, since sparsity is a central pillar of not just $\mathrm{CS}$, but much of modern signal processing. However, this can be confirmed by a simple experiment we refer to as the flip test. 
Sparsity asserts that an unknown vector $x$ has $s$ important coefficients, where the locations can be arbitrary, and classical CS theory states that such vectors can be recovered by sampling in a way that is independent of the locations of these coefficients. The flip test, described next, allows one to evaluate the validity of this statement for a given problem.

We proceed as follows. Let $x \in \mathbb{C}^{N}$ and $U \in \mathbb{C}^{N \times N}$. Take samples according to some appropriate subset $\Omega \subseteq\{1, \ldots, N\}$ with $|\Omega|=m$, and solve:

$$
\min _{z \in \mathbb{C}^{N}}\|z\|_{1} \text { subject to } P_{\Omega} U z=P_{\Omega} U x \text {. }
$$

This gives a reconstruction $z=z_{1}$. Now we flip the entries of $x$ through the operation $x \mapsto x^{\mathrm{fp}} \in \mathbb{C}^{N}, x_{1}^{\mathrm{fp}}=x_{N}, x_{2}^{\mathrm{fp}}=x_{N-1}, \ldots, x_{N}^{\mathrm{fp}}=x_{1}$, giving a new vector $x^{\mathrm{fp}}$ with reversed entries. We next apply the same CS reconstruction to $x^{\mathrm{fp}}$, using the same matrix $U$ and the same subset $\Omega$. That is, we solve (2.6) with $x$ replaced by $x^{\mathrm{fp}}$ and denote the solution by $z$. We perform the flipping operation once more and form the final reconstruction $z_{2}=z^{\mathrm{fp}}$.

Suppose now that $\Omega$ is a good sampling pattern for recovering $x$ using the solution $z_{1}$ of (2.6). If sparsity alone is the key structure that determines such reconstruction quality, then we expect roughly the same quality in the approximation $z_{2}$, since $x^{\mathrm{fp}}$ is merely a permutation of $x$. To check whether or not this is true, we consider examples arising from the following applications: fluorescence microscopy, compressive imaging, MRI, CT, electron microscopy and radio interferometry. These examples are all based on either the matrix $U=U_{\mathrm{dft}} V_{\mathrm{dwt}}^{-1}$ or the matrix $U=U_{\mathrm{Had}} V_{\mathrm{dwt}}^{-1}$, where $U_{\mathrm{dft}}$ is the discrete Fourier transform, $U_{\text {Had }}$ is a Hadamard matrix and $V_{\mathrm{dwt}}$ is the discrete wavelet transform.

The results are shown in Figure 2. In all cases the flipped reconstructions $z_{2}$ are substantially worse than their unflipped counterparts $z_{1}$. We therefore conclude that sparsity alone does not govern the reconstruction quality, and that the successful recovery in the unflipped case must also be due in part to the structure of the signal. Put another way: the best subsampling strategy depends on the signal structure.

The flip test also reveals another interesting phenomenon: the RIP does not hold. Suppose the matrix $P_{\Omega} U$ satisfied an RIP for realistic parameter values (that is problem size $N$, subsampling percentage $m$, and sparsity $s$ ) found in applications. Then this would imply recovery of all approximately sparse vectors with the same error, in contradiction with the results of the flip test. As was mentioned in Section 1.1, the absence of RIP here is not related to uniform versus nonuniform recovery regimes, but to the key role that the sparsity structure plays in the recovery quality. Indeed, the result of Figure 2 could have been repeated with more measurements and similar disparities in the reconstruction quality would still have been observed. 
CS reconstruction

$512 \times 512$

$10 \%$

$U_{\mathrm{Had}} \cdot V_{\mathrm{dwt}}^{-1}$

Fluorescence

Microscopy

$512 \times 512$

$15 \%$

$U_{\mathrm{Had}} \cdot V_{\mathrm{dwt}}^{-1}$

Compressive

Imaging

$1024 \times 1024$

$20 \%$

$U_{\mathrm{dft}} \cdot V_{\mathrm{dwt}}^{-1}$

MRI

$512 \times 512$

$12 \%$

$U_{\mathrm{dft}} \cdot V_{\mathrm{dwt}}^{-1}$

Tomography

$512 \times 512$

$15 \%$

$U_{\mathrm{dft}} \cdot V_{\mathrm{dwt}}^{-1}$

Radio

interferometry

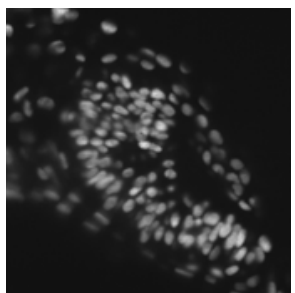

CS reconstruction w/ flip

Subsampling pattern
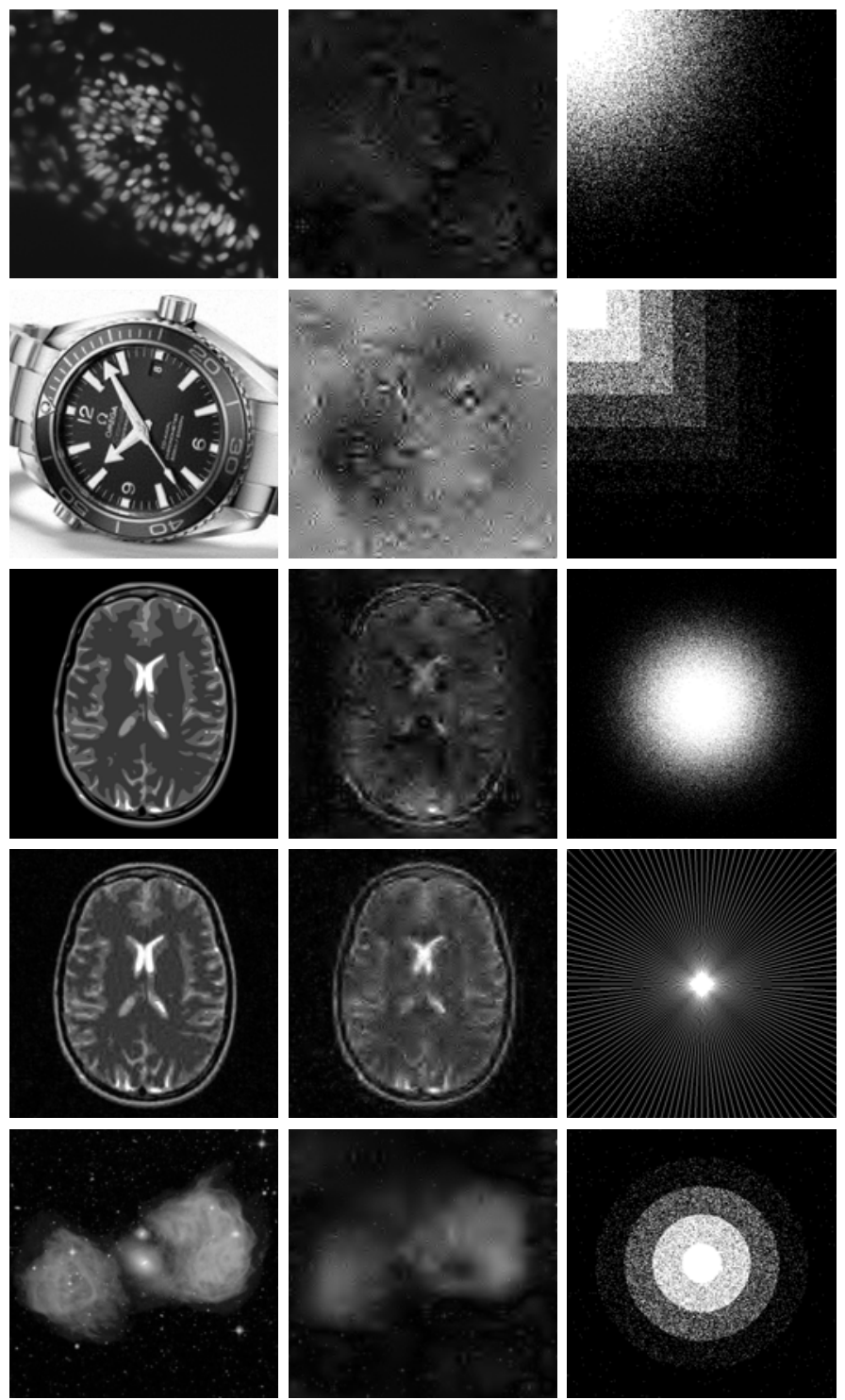

Figure 2. Reconstructions via CS (left column) and the flipped wavelet coefficients (middle column). The right column shows the subsampling map used. The percentage shown is the fraction of Fourier or Hadamard coefficients that were sampled. The reconstruction basis was DB6. 
2.4. Signals and images are asymptotically sparse in -lets. Since structure plays a key role, we now address what the structure is that leads to good reconstructions in the unflipped case. Consider a wavelet basis $\left\{\varphi_{n}\right\}_{n \in \mathbb{N}}$. There is a natural decomposition of $\mathbb{N}$ into finite subsets according to the wavelet scales, $\mathbb{N}=\bigcup_{k \in \mathbb{N}}\left\{M_{k-1}+1, \ldots, M_{k}\right\}$, where $0=M_{0}<M_{1}<M_{2}<\ldots$ and $\left\{M_{k-1}+1\right.$, $\left.\ldots, M_{k}\right\}$ is the set of indices corresponding to the $k$ th scale. Let $x \in l^{2}(\mathbb{N})$ be the coefficients of a function $f$ in this basis, $\epsilon \in(0,1]$ and define the global sparsity, $s$, and the sparsity at the $k$ th level, $s_{k}$ as follows:

$$
\begin{aligned}
& s=s(\epsilon)=\min \left\{n:\left\|\sum_{i \in \mathcal{M}_{n}} x_{i} \varphi_{i}\right\| \geqslant \epsilon\left\|\sum_{j=1}^{\infty} x_{j} \varphi_{j}\right\|\right\}, \\
& s_{k}=s_{k}(\epsilon)=\left|\mathcal{M}_{s(\epsilon)} \cap\left\{M_{k-1}+1, \ldots, M_{k}\right\}\right|,
\end{aligned}
$$

where $\mathcal{M}_{n}$ is the set of indices of the largest $n$ coefficients in absolute value and $|\cdot|$ is the set cardinality. A well-known result in nonlinear approximation, and one which significantly predates the development of CS, is that typical images and signals are sparse in wavelets $[30,64]$. However, it is also well known that their coefficients exhibit far more structure than sparsity alone. Indeed, the relative perlevel sparsity

$$
s_{k} /\left(M_{k}-M_{k-1}\right) \longrightarrow 0,
$$

rapidly as $k \rightarrow \infty$ for any fixed $\epsilon \in(0,1]$. Thus typical signals and images have a distinct asymptotic sparsity structure: they are much sparser at fine scales (large $k$ ) than at coarse scales (small $k$ ). This is shown numerically in Figure 3. Note that this holds for most related approximation systems, such as curvelets [13, 15], contourlets $[31,66]$ or shearlets $[25,26,56]$.

\section{New principles}

Having argued why they are needed, we now formally introduce the main concepts of the paper: namely, asymptotic incoherence, asymptotic sparsity and multilevel sampling.

3.1. Asymptotic incoherence. Recall from Section 2.2 that Fourier sampling with wavelets as the sparsity basis is a standard example of a coherent problem. Similarly, Fourier sampling with Legendre polynomials is also coherent, as is the case of Hadamard sampling with wavelets. In Figure 4 we plot the absolute values of the entries of the matrix $U$ for these three examples. As is evident, whilst $U$ does indeed have large entries in all three case (since it is coherent), these are isolated to a leading submatrix (note that we enumerate over $\mathbb{Z}$ for the Fourier 

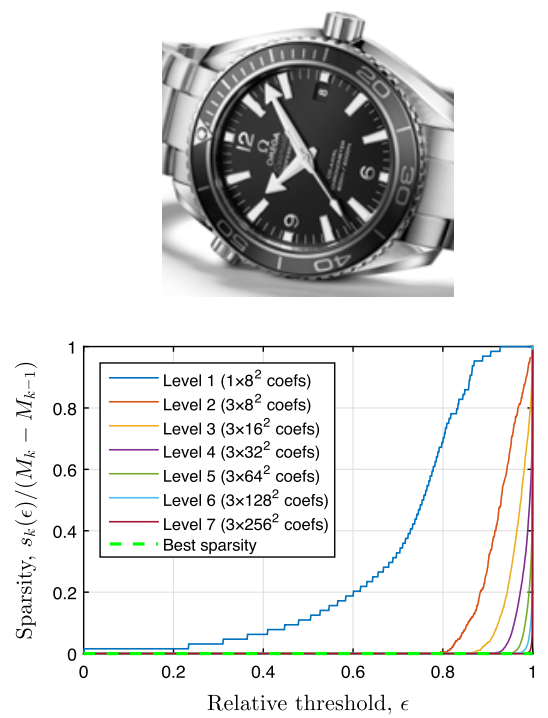
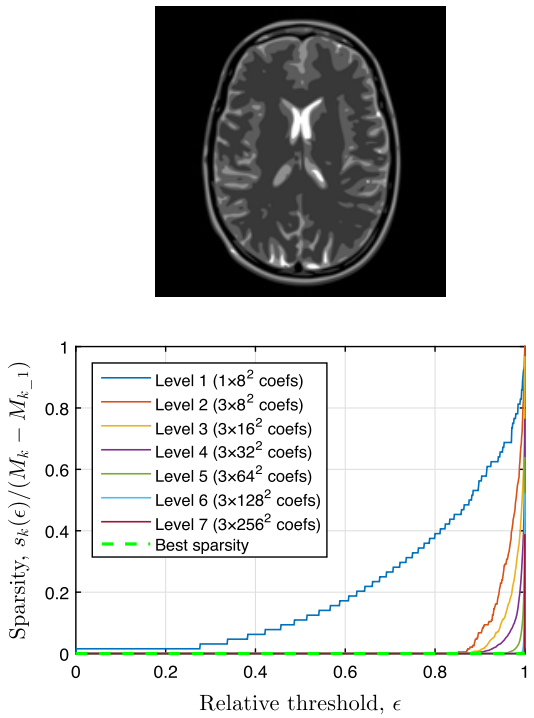

Figure 3. Relative per-level sparsities of the Daubechies-4 wavelet coefficients of two images. Here the levels correspond to wavelet scales and $s_{k}(\epsilon)$ is given by (2.7). The decreasing nature of the curves for increasing $k$ confirms (2.8).
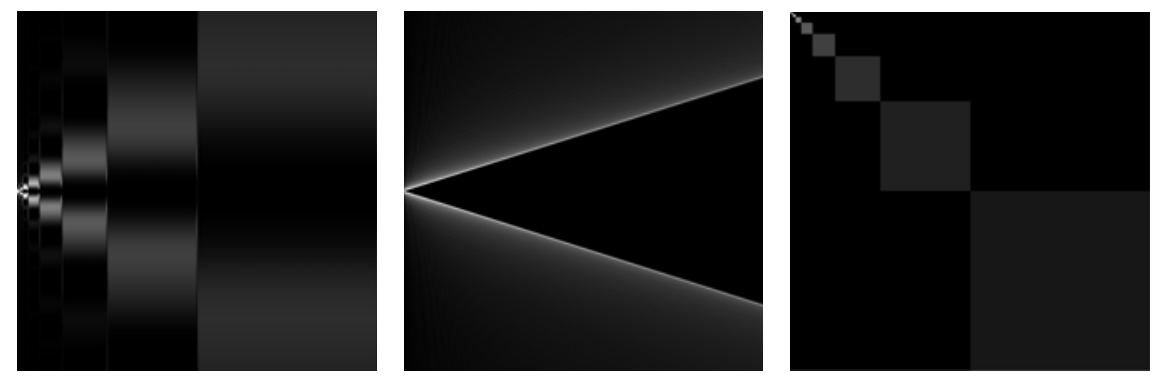

Figure 4. The absolute values of the matrix $U$ in (2.5): (left): Daubechies-4 wavelets with Fourier sampling. (middle): Legendre polynomials with Fourier sampling. (right): Haar wavelets with Hadamard sampling. Light regions correspond to large values and dark regions to small values.

sampling basis and $\mathbb{N}$ for the wavelet/Legendre sparsity bases). As one moves away from this region the values get progressively smaller, that is more incoherent. This motivates the following definition: 
DEFINITION 3.1 (Asymptotic incoherence). Let be $\left\{U_{N}\right\}$ be a sequence of isometries with $U_{N} \in \mathbb{C}^{N \times N}$. Then $\left\{U_{N}\right\}$ is asymptotically incoherent if

$$
\mu\left(P_{K}^{\perp} U_{N}\right), \mu\left(U_{N} P_{K}^{\perp}\right) \longrightarrow 0, \quad K \rightarrow \infty,
$$

with $N / K=c$, for all $c \geqslant 1$. Conversely, if $U \in \mathcal{B}\left(l^{2}(\mathbb{N})\right)$ then we say that $U$ is asymptotically incoherent if $\mu\left(P_{K}^{\perp} U\right), \mu\left(U P_{K}^{\perp}\right) \rightarrow 0$ when $K \rightarrow \infty$.

Note that in this definition we use the notation $P_{K}$ for the projection onto $\operatorname{span}\left\{e_{j}: j=1, \ldots, K\right\}$, where $\left\{e_{j}\right\}$ is the canonical basis of either $\mathbb{C}^{N}$ or $l^{2}(\mathbb{N})$, and $P_{K}^{\perp}$ is its orthogonal complement.

In other words, $U$ (or $U_{N}$ ) is asymptotically incoherent if the coherences of the matrices formed by replacing either the first $K$ rows or columns of $U$ are small. As it transpires, the Fourier-wavelets, Fourier-Legendre and Hadamard-wavelets problems are all asymptotically incoherent. In particular, for wavelets one has $\mu\left(P_{K}^{\perp} U\right), \mu\left(U P_{K}^{\perp}\right)=\mathcal{O}\left(K^{-1}\right)$ as $K \rightarrow \infty$ for the former (see Section 6, see also [54] for the Legendre case).

3.2. Multilevel sampling. Asymptotic incoherence suggests a different subsampling strategy should be used instead of uniform random sampling. High coherence in the first few rows of $U$ means that important information about the signal to be recovered may well be contained in its corresponding measurements. Hence to ensure good recovery we should fully sample these rows. Conversely, once outside of this region, when the coherence starts to decrease, we can begin to subsample. Let $N_{1}, N, m \in \mathbb{N}$ be given. This now leads us to consider an index set $\Omega$ of the form $\Omega=\Omega_{1} \cup \Omega_{2}$, where $\Omega_{1}=\left\{1, \ldots, N_{1}\right\}$, and $\Omega_{2} \subseteq\left\{N_{1}+1, \ldots, N\right\}$ is chosen uniformly at random with $\left|\Omega_{2}\right|=m$. We refer to this as a two-level sampling scheme. As we shall prove later, the amount of subsampling possible (that is the parameter $m$ ) in the region corresponding to $\Omega_{2}$ will depend solely on the sparsity of the signal and coherence $\mu\left(P_{N_{1}}^{\perp} U\right)$.

A two-level scheme represents the simplest type of nonuniform density sampling. There is no reason, however, to restrict our attention to just two levels, full and subsampled. In general, we shall consider multilevel schemes, defined as follows:

DEFINITION 3.2 (Multilevel random sampling). Let $r \in \mathbb{N}, \mathbf{N}=\left(N_{1}, \ldots, N_{r}\right)$ $\in \mathbb{N}^{r}$ with $1 \leqslant N_{1}<\cdots<N_{r}, \mathbf{m}=\left(m_{1}, \ldots, m_{r}\right) \in \mathbb{N}^{r}$, with $m_{k} \leqslant N_{k}-N_{k-1}$, $k=1, \ldots, r$, and suppose that $\Omega_{k} \subseteq\left\{N_{k-1}+1, \ldots, N_{k}\right\},\left|\Omega_{k}\right|=m_{k}$, $k=1, \ldots, r$, are chosen uniformly at random, where $N_{0}=0$. We refer to the set $\Omega=\Omega_{\mathbf{N}, \mathbf{m}}=\Omega_{1} \cup \cdots \cup \Omega_{r}$ as an $(\mathbf{N}, \mathbf{m})$-multilevel sampling scheme. 
As discussed earlier, two-level $[32,63,74,77]$ and multilevel $[17,82]$ schemes have been considered previously in the context of the recovery of wavelet coefficients, and often for specific applications (for example, MRI in [63] and fluorescence microscopy in [77]). We refer to Section 1.2 for further details. On the other hand, although motivated by wavelets, this definition is completely general and allows for other types of structured coefficients. Moreover, it is accompanied by full theoretical recovery guarantees (see Sections 4 and 5).

3.3. Asymptotic sparsity in levels. The flip test, the discussion in Section 2.4 and Figure 3 suggest that we need a different concept to sparsity. Given the structure of function systems such as wavelets and their generalizations, we now propose the following:

Definition 3.3 (Sparsity in levels). Let $x$ be an element of either $\mathbb{C}^{N}$ or $l^{2}(\mathbb{N})$. For $r \in \mathbb{N}$ let $\mathbf{M}=\left(M_{1}, \ldots, M_{r}\right) \in \mathbb{N}^{r}$ with $1 \leqslant M_{1}<\cdots<M_{r}$ and $\mathbf{s}=$ $\left(s_{1}, \ldots, s_{r}\right) \in \mathbb{N}^{r}$, with $s_{k} \leqslant M_{k}-M_{k-1}, k=1, \ldots, r$, where $M_{0}=0$. We say that $x$ is (s, M)-sparse if, for each $k=1, \ldots, r, \Delta_{k}:=\operatorname{supp}(x) \cap\left\{M_{k-1}+1, \ldots, M_{k}\right\}$, satisfies $\left|\Delta_{k}\right| \leqslant s_{k}$. We denote the set of $(\mathbf{s}, \mathbf{M})$-sparse vectors by $\Sigma_{\mathbf{s}, \mathbf{M}}$.

Definition 3.4 ((s, M)-term approximation). Let $f=\sum_{j} x_{j} \varphi_{j}$, where $\left\{\varphi_{j}\right\}$ is some orthonormal basis of a Hilbert space and $x=\left(x_{j}\right)$ is an element of either $\mathbb{C}^{N}$ or $l^{2}(\mathbb{N})$. We define the $(\mathbf{s}, \mathbf{M})$-term approximation

$$
\sigma_{\mathbf{s}, \mathbf{M}}(f)=\min _{\eta \in \Sigma_{\mathbf{s}, \mathbf{M}}}\|x-\eta\|_{l^{1}}
$$

Typically, it is the case that $s_{k} /\left(M_{k}-M_{k-1}\right) \rightarrow 0$ as $k \rightarrow \infty$, in which case we say that $x$ is asymptotically sparse in levels. However, our main results do not explicitly require such decay. As discussed in Section 1.2, sparsity in levels is a type of structured sparsity. We note in passing that it is quite different to the notions of block sparsity [8], weighted sparsity [72] or tree-structured sparsity [8], the latter of which has been used previously in the context of model-based CS for the recovery of wavelet coefficients. For further discussion on different structured sparsity models in CS, we refer to $[\mathbf{1 1 , 8 0 ] .}$

\section{Main theorems I: the finite-dimensional case}

We now present our main theorems in the finite-dimensional setting. In Section 5 we address the infinite-dimensional case. To avoid pathological examples we assume throughout that the total sparsity $s=s_{1}+\cdots+s_{r} \geqslant 3$. This is simply to ensure that $\log (s) \geqslant 1$, which is convenient in the proofs. 
4.1. Two-level sampling schemes. We commence with the case of two-level sampling schemes. Recall that in practice, signals are never exactly sparse (or sparse in levels), and their measurements are always contaminated by noise. Let $f=\sum_{j} x_{j} \varphi_{j}$ be a fixed signal, and write $y=P_{\Omega} \hat{f}+z=P_{\Omega} U x+z$, for its noisy measurements, where $z \in \operatorname{ran}\left(P_{\Omega}\right)$ is a noise vector satisfying $\|z\| \leqslant \delta$ for some $\delta \geqslant 0$. If $\delta$ is known, we now consider the following problem:

$$
\min _{\eta \in \mathbb{C}^{N}}\|\eta\|_{l^{1}} \text { subject to }\left\|P_{\Omega} U \eta-y\right\| \leqslant \delta .
$$

Our aim now is to recover $x$ up to an error proportional to $\delta$ and the best approximation error $\sigma_{\mathbf{s}, \mathbf{M}}(f)$.

Before stating our theorem, it is useful to make the following definition. For $K \in \mathbb{N}$, we write $\mu_{K}=\mu\left(P_{K}^{\perp} U\right)$. We now have the following:

THEOREM 4.1. Let $U \in \mathbb{C}^{N \times N}$ be an isometry and $x \in \mathbb{C}^{N}$. Suppose that $\Omega=\Omega_{\mathbf{N}, \mathbf{m}}$ is a two-level sampling scheme, where $\mathbf{N}=\left(N_{1}, N_{2}\right), N_{2}=N$, and $\mathbf{m}=\left(N_{1}, m_{2}\right)$. Let $(\mathbf{s}, \mathbf{M})$, where $\mathbf{M}=\left(M_{1}, M_{2}\right) \in \mathbb{N}^{2}, M_{1}<M_{2}, M_{2}=N$, and $\mathbf{s}=\left(M_{1}, s_{2}\right) \in \mathbb{N}^{2}, s_{2} \leqslant M_{2}-M_{1}$, be any pair such that the following holds:

(i) we have

$$
\left\|P_{N_{1}}^{\perp} U P_{M_{1}}\right\| \leqslant \frac{\gamma}{\sqrt{M_{1}}}
$$

and $\gamma \leqslant s_{2} \sqrt{\mu_{N_{1}}}$ for some $\gamma \in(0,2 / 5]$;

(ii) for $\epsilon \in\left(0, e^{-1}\right]$, let

$$
m_{2} \gtrsim\left(N-N_{1}\right) \cdot \log \left(\epsilon^{-1}\right) \cdot \mu_{N_{1}} \cdot s_{2} \cdot \log (N) .
$$

Suppose that $\xi \in \mathbb{C}^{N}$ is a minimizer of (4.1) with $\delta=\tilde{\delta} \sqrt{K^{-1}}$ and $K=$ $\left(N_{2}-N_{1}\right) / m_{2}$. Then, with probability exceeding $1-s \epsilon$, we have

$$
\|\xi-x\| \leqslant C \cdot\left(\tilde{\delta} \cdot(1+L \cdot \sqrt{s})+\sigma_{\mathbf{s}, \mathbf{M}}(f)\right),
$$

for some constant $C$, where $\sigma_{\mathbf{s}, \mathbf{M}}(f)$ is as in $(3.1), L=1+\left(\sqrt{\log _{2}\left(6 \epsilon^{-1}\right)} /\right.$ $\left.\log _{2}(4 K M \sqrt{s})\right)$. If $m_{2}=N-N_{1}$ then this holds with probability 1 .

To interpret Theorem 4.1, and in particular, to show how it overcomes the coherence barrier, we note the following:

(i) The condition $\left\|P_{N_{1}}^{\perp} U P_{M_{1}}\right\| \leqslant 2 /\left(5 \sqrt{M_{1}}\right)$ (which is always satisfied for some $N_{1}$ ) implies that fully sampling the first $N_{1}$ measurements allows one to recover the first $M_{1}$ coefficients of $f$. 
(ii) To recover the remaining $s_{2}$ coefficients we require, up to log factors, an additional $m_{2} \gtrsim\left(N-N_{1}\right) \cdot \mu_{N_{1}} \cdot s_{2}$, measurements, taken randomly from the range $M_{1}+1, \ldots, M_{2}$. In particular, if $N_{1}$ is a fixed fraction of $N$, and if $\mu_{N_{1}}=\mathcal{O}\left(N_{1}^{-1}\right)$, such as for wavelets with Fourier measurements (Theorem 6.1), then one requires only $m_{2} \gtrsim s_{2}$ additional measurements to recover the sparse part of the signal.

Thus, in the case where $x$ is asymptotically sparse, we require a fixed number $N_{1}$ measurements to recover the nonsparse part of $x$, and then a number $m_{2}$ depending on $s_{2}$ and the asymptotic coherence $\mu_{N_{1}}$ to recover the sparse part.

REMARK 4.2. It is not necessary to know the sparsity structure, that is the values $\mathbf{S}$ and $\mathbf{M}$, of the signal $f$ in order to implement the two-level sampling technique (the same also applies to the multilevel technique discussed in the next section). Given a two-level scheme $\Omega=\Omega_{\mathbf{N}, \mathbf{m}}$, Theorem 4.1 demonstrates that $f$ will be recovered exactly up to an error on the order of $\sigma_{\mathbf{s}, \mathbf{M}}(f)$, where $\mathbf{s}$ and $\mathbf{M}$ are determined implicitly by $\mathbf{N}, \mathbf{m}$ and the conditions (i) and (ii) of the theorem. Of course, some a priori knowledge of $\mathbf{s}$ and $\mathbf{M}$ will greatly assist in selecting the parameters $\mathbf{N}$ and $\mathbf{m}$ so as to get the best recovery results. However, this is not strictly necessary for implementation.

4.2. Multilevel sampling schemes. We now consider the case of multilevel sampling schemes. Before presenting this case, we need several definitions. The first is key concept in this paper, namely, the local coherence.

DEFINITION 4.3 (Local coherence). Let $U$ be an isometry of either $\mathbb{C}^{N}$ or $l^{2}(\mathbb{N})$. If $\mathbf{N}=\left(N_{1}, \ldots, N_{r}\right) \in \mathbb{N}^{r}$ and $\mathbf{M}=\left(M_{1}, \ldots, M_{r}\right) \in \mathbb{N}^{r}$ with $1 \leqslant N_{1}<\cdots N_{r}$ and $1 \leqslant M_{1}<\cdots<M_{r}$ the $(k, l)$ th local coherence of $U$ with respect to $\mathbf{N}$ and $\mathbf{M}$ is given by

$$
\mu_{\mathbf{N}, \mathbf{M}}(k, l)=\sqrt{\mu\left(P_{N_{k}}^{N_{k-1}} U P_{M_{l}}^{M_{l-1}}\right) \cdot \mu\left(P_{N_{k}}^{N_{k-1}} U\right)}, \quad k, l=1, \ldots, r,
$$

where $N_{0}=M_{0}=0$ and $P_{b}^{a}$ denotes the projection matrix corresponding to indices $\{a+1, \ldots, b\}$. In the case where $U \in \mathcal{B}\left(l^{2}(\mathbb{N})\right)$ (that is $U$ belongs to the space of bounded operators on $\left.l^{2}(\mathbb{N})\right)$, we also define

$$
\mu_{\mathbf{N}, \mathbf{M}}(k, \infty)=\sqrt{\mu\left(P_{N_{k}}^{N_{k-1}} U P_{M_{r-1}}^{\perp}\right) \cdot \mu\left(P_{N_{k}}^{N_{k-1}} U\right)}, \quad k=1, \ldots, r .
$$

Besides the local sparsities $s_{k}$, we shall also require the notion of a relative sparsity: 
Definition 4.4 (Relative sparsity). Let $U$ be an isometry of either $\mathbb{C}^{N}$ or $l^{2}(\mathbb{N})$. For $\mathbf{N}=\left(N_{1}, \ldots, N_{r}\right) \in \mathbb{N}^{r}, \mathbf{M}=\left(M_{1}, \ldots, M_{r}\right) \in \mathbb{N}^{r}$ with $1 \leqslant N_{1}<\cdots<N_{r}$ and $1 \leqslant M_{1}<\cdots<M_{r}, \mathbf{s}=\left(s_{1}, \ldots, s_{r}\right) \in \mathbb{N}^{r}$ and $1 \leqslant k \leqslant r$, the $k$ th relative sparsity is given by $S_{k}=S_{k}(\mathbf{N}, \mathbf{M}, \mathbf{s})=\max _{\eta \in \Theta}\left\|P_{N_{k}}^{N_{k-1}} U \eta\right\|^{2}$, where $N_{0}=M_{0}=0$ and $\Theta$ is the set

$$
\Theta=\left\{\eta:\|\eta\|_{l^{\infty}} \leqslant 1,\left|\operatorname{supp}\left(P_{M_{l}}^{M_{l-1}} \eta\right)\right|=s_{l}, l=1, \ldots, r\right\} .
$$

We can now present our main theorem:

THEOREM 4.5. Let $U \in \mathbb{C}^{N \times N}$ be an isometry and $x \in \mathbb{C}^{N}$. Suppose that $\Omega=\Omega_{\mathbf{N}, \mathbf{m}}$ is a multilevel sampling scheme, where $\mathbf{N}=\left(N_{1}, \ldots, N_{r}\right) \in \mathbb{N}^{r}$, $N_{r}=N$, and $\mathbf{m}=\left(m_{1}, \ldots, m_{r}\right) \in \mathbb{N}^{r}$. Let $(\mathbf{s}, \mathbf{M})$, where $\mathbf{M}=\left(M_{1}, \ldots, M_{r}\right) \in \mathbb{N}^{r}$, $M_{r}=N$, and $\mathbf{s}=\left(s_{1}, \ldots, s_{r}\right) \in \mathbb{N}^{r}$, be any pair such that the following holds: for $\epsilon \in\left(0, e^{-1}\right]$ and $1 \leqslant k \leqslant r$,

$$
1 \gtrsim \frac{N_{k}-N_{k-1}}{m_{k}} \cdot \log \left(\epsilon^{-1}\right) \cdot\left(\sum_{l=1}^{r} \mu_{\mathbf{N}, \mathbf{M}}(k, l) \cdot s_{l}\right) \cdot \log (N),
$$

where $m_{k} \gtrsim \hat{m}_{k} \cdot \log \left(\epsilon^{-1}\right) \cdot \log (N)$, and $\hat{m}_{k}$ is such that

$$
1 \gtrsim \sum_{k=1}^{r}\left(\frac{N_{k}-N_{k-1}}{\hat{m}_{k}}-1\right) \cdot \mu_{\mathbf{N}, \mathbf{M}}(k, l) \cdot \tilde{s}_{k},
$$

for all $l=1, \ldots, r$ and all $\tilde{s}_{1}, \ldots, \tilde{s}_{r} \in(0, \infty)$ satisfying

$$
\tilde{s}_{1}+\cdots+\tilde{s}_{r} \leqslant s_{1}+\cdots+s_{r}, \quad \tilde{s}_{k} \leqslant S_{k}(\mathbf{N}, \mathbf{M}, \mathbf{s}) .
$$

Suppose that $\xi \in \mathbb{C}^{N}$ is a minimizer of (4.1) with $\delta=\tilde{\delta} \sqrt{K^{-1}}$ and $K=\max _{1 \leqslant k \leqslant r}\left\{\left(N_{k}-N_{k-1}\right) / m_{k}\right\}$. Then, with probability exceeding $1-s \epsilon$, where $s=s_{1}+\cdots+s_{r}$, we have that

$$
\|\xi-x\| \leqslant C \cdot\left(\tilde{\delta} \cdot(1+L \cdot \sqrt{s})+\sigma_{\mathbf{s}, \mathbf{M}}(f)\right),
$$

for some constant $C$, where $\sigma_{\mathbf{s}, \mathbf{M}}(f)$ is as in $(3.1), L=1+\left(\sqrt{\log _{2}\left(6 \epsilon^{-1}\right)} /\right.$ $\left.\log _{2}(4 K M \sqrt{s})\right)$. If $m_{k}=N_{k}-N_{k-1}, 1 \leqslant k \leqslant r$, then this holds with probability 1 .

The key component of this theorem is the bounds (4.4) and (4.5). Whereas the standard CS estimate (2.2) relates the total number of samples $m$ to the global coherence and the global sparsity, these bounds now relate the local sampling 
$m_{k}$ to the local coherences $\mu_{\mathbf{N}, \mathbf{M}}(k, l)$ and local and relative sparsities $s_{k}$ and $S_{k}$. In particular, by relating these local quantities this theorem conforms with the conclusions of the flip test in Section 2.3: namely, that the optimal sampling strategy must depend on the signal structure. This is exactly what is described in (4.4) and (4.5).

On the face of it, the bounds (4.4) and (4.5) may appear somewhat complicated, not least because they involve the relative sparsities $S_{k}$. As we next show, however, they are indeed sharp in the sense that they reduce to the correct informationtheoretic limits in several important cases. Furthermore, in the important case of wavelet sparsity with Fourier sampling, they can be used to provide near-optimal recovery guarantees. We discuss this in Section 6. Note, however, that to do this it is first necessary to generalize Theorem 4.5 to the infinite-dimensional setting, which we do in Section 5.

4.2.1. Sharpness of the estimates-the block-diagonal case. Suppose that $\Omega=\Omega_{\mathbf{N}, \mathbf{m}}$ is a multilevel sampling scheme, where $\mathbf{N}=\left(N_{1}, \ldots, N_{r}\right) \in \mathbb{N}^{r}$ and $\mathbf{m}=\left(m_{1}, \ldots, m_{r}\right) \in \mathbb{N}^{r}$. Let $(\mathbf{s}, \mathbf{M})$, where $\mathbf{M}=\left(M_{1}, \ldots, M_{r}\right) \in \mathbb{N}^{r}$, and suppose for simplicity that $\mathbf{M}=\mathbf{N}$. Consider the block-diagonal matrix

$$
A=A_{1} \oplus \cdots \oplus A_{r} \in \mathbb{C}^{N \times N}, \quad A_{k} \in \mathbb{C}^{\left(N_{k}-N_{k-1}\right) \times\left(N_{k}-N_{k-1}\right)}, \quad A_{k}^{*} A_{k}=I,
$$

where $N_{0}=0$. Note that in this setting we have $S_{k}=s_{k}, \mu_{\mathbf{N}, \mathbf{M}}(k, l)=0, k \neq l$. Also, since $\mu(\mathbf{N}, \mathbf{M})(k, k)=\mu\left(A_{k}\right)$, equations (4.4) and (4.5) reduce to

$$
\begin{gathered}
1 \gtrsim \frac{N_{k}-N_{k-1}}{m_{k}} \cdot \log \left(\epsilon^{-1}\right) \cdot \mu\left(A_{k}\right) \cdot s_{k} \cdot \log (N), \\
1 \gtrsim\left(\frac{N_{k}-N_{k-1}}{\hat{m}_{k}}-1\right) \cdot \mu\left(A_{k}\right) \cdot s_{k} .
\end{gathered}
$$

In particular, it suffices to take

$$
m_{k} \gtrsim\left(N_{k}-N_{k-1}\right) \cdot \log \left(\epsilon^{-1}\right) \cdot \mu\left(A_{k}\right) \cdot s_{k} \cdot \log (N), \quad 1 \leqslant k \leqslant r .
$$

This is exactly as one expects: the number of measurements in the $k$ th level depends on the size of the level multiplied by the local coherence and the sparsity in that level. Note that this result recovers the standard one-level results in finite dimensions $[3,16]$ up to a slight deterioration in the probability bound to $1-s \epsilon$. Specifically, the usual bound would be $1-\epsilon$. The question as to whether or not this $s$ can be removed in the multilevel setting is open, although such a result would be more of a cosmetic improvement. 
4.2.2. Sharpness of the estimates-the nonblock-diagonal case. The previous argument demonstrated that Theorem 4.5 is sharp, up to the probability term, in the sense that it reduces to the usual estimate (4.6) for block-diagonal matrices, $S_{k}=s_{k}$. This is not true in the general setting. Clearly, $S_{k} \leqslant s=s_{1}+\cdots+s_{r}$. However, in general there is usually interference between different sparsity levels, which means that $S_{k}$ need not have anything to do with $s_{k}$, or can indeed be proportional to the total sparsity $s$. This may seem an undesirable aspect of the theorems, since $S_{k}$ may be significantly larger than $s_{k}$, and thus the estimate on the number of measurements $m_{k}$ required in the $k$ th level may also be much larger than the corresponding sparsity $s_{k}$. Could it therefore be that the $S_{k}$,s are an unfortunate artefact of the proof? As we now show by example, this is not the case.

Let $N=r n$ for some $n \in \mathbb{N}$ and $\mathbf{N}=\mathbf{M}=(n, 2 n, \ldots, r n)$. Let $W \in \mathbb{C}^{n \times n}$ and $V \in \mathbb{C}^{r \times r}$ be isometries and consider the matrix

$$
A=V \otimes W
$$

where $\otimes$ is the usual Kronecker product. Note that $A \in \mathbb{C}^{N \times N}$ is also an isometry. Now suppose that $x=\left(x_{1}, \ldots, x_{r}\right) \in \mathbb{C}^{N}$ is an (s, M)-sparse vector, where each $x_{k} \in \mathbb{C}^{n}$ is $s_{k}$-sparse. Then $A x=y, y=\left(y_{1}, \ldots, y_{r}\right), y_{k}=W z_{k}$, $z_{k}=\sum_{l=1}^{r} v_{k l} x_{l}$. Hence the problem of recovering $x$ from measurements $y$ with an $(\mathbf{N}, \mathbf{m})$-multilevel strategy decouples into $r$ problems of recovering the vector $z_{k}$ from the measurements $y_{k}=W z_{k}, k=1, \ldots, r$. Let $\hat{s}_{k}$ denote the sparsity of $z_{k}$. Since the coherence provides an information-theoretic limit [16], one requires at least

$$
m_{k} \gtrsim n \cdot \mu(W) \cdot \hat{s}_{k} \cdot \log (n), \quad 1 \leqslant k \leqslant r
$$

measurements at level $k$ in order to recover each $z_{k}$, and therefore recover $x$, regardless of the reconstruction method used. We now consider two examples of this setup:

EXAMPLE 4.6. Let $\pi:\{1, \ldots, r\} \rightarrow\{1, \ldots, r\}$ be a permutation and let $V$ be the matrix with entries $v_{k l}=\delta_{l, \pi(k)}$. Since $z_{k}=x_{\pi(k)}$ in this case, the lower bound (4.7) reads

$$
m_{k} \gtrsim n \cdot \mu(W) \cdot s_{\pi(k)} \cdot \log (n), \quad 1 \leqslant k \leqslant r .
$$

Now consider Theorem 4.5 for this matrix. First, we note that $S_{k}=s_{\pi(k)}$. In particular, $S_{k}$ is completely unrelated to $s_{k}$. Substituting this into Theorem 4.5 and noting that $\mu_{\mathbf{N}, \mathbf{M}}(k, l)=\mu(W) \delta_{l, \pi(k)}$ in this case, we arrive at the condition $m_{k} \gtrsim n \cdot \mu(W) \cdot s_{\pi(k)} \cdot\left(\log \left(\epsilon^{-1}\right)+1\right) \cdot \log (n r)$, which is equivalent to (4.8) provided $r \lesssim n$. 
EXAMPLE 4.7. Now suppose that $V$ is the $r \times r$ DFT matrix. Suppose also that $s \leqslant n / r$ and that the $x_{k}$ 's have disjoint support sets, that is $\operatorname{supp}\left(x_{k}\right) \cap \operatorname{supp}\left(x_{l}\right)=\emptyset$, $k \neq l$. Then by construction, each $z_{k}$ is $s$-sparse, and therefore the lower bound (4.7) reads $m_{k} \gtrsim n \cdot \mu(W) \cdot s \cdot \log n$, for $1 \leqslant k \leqslant r$. After a short argument, one finds that $s / r \leqslant S_{k} \leqslant s$ in this case. Hence, $S_{k}$ is typically much larger than $s_{k}$. Moreover, after noting that $\mu_{\mathbf{N}, \mathbf{M}}(k, l)=(1 / r) \mu(W)$, we find that Theorem 4.5 gives the condition $m_{k} \gtrsim n \cdot \mu(W) \cdot s \cdot\left(\log \left(\epsilon^{-1}\right)+1\right) \cdot \log (n r)$. Thus, Theorem 4.5 obtains the lower bound in this case as well.

4.2.3. Sparsity leads to pessimistic reconstruction guarantees. The flip test demonstrates that any sparsity-based theory of CS cannot describe the quality of the reconstructions seen in practice. To conclude this section, we now use the block-diagonal case to further emphasize the need for theorems that go beyond sparsity, such as Theorems 4.1 and 4.5. To see this, consider the block-diagonal matrix

$$
U=U_{1} \oplus \cdots \oplus U_{r}, \quad U_{k} \in \mathbb{C}^{\left(N_{k}-N_{k-1}\right) \times\left(N_{k}-N_{k-1}\right)},
$$

where each $U_{k}$ is perfectly incoherent, that is $\mu\left(U_{k}\right)=\left(N_{k}-N_{k-1}\right)^{-1}$, and suppose we take $m_{k}$ measurements within each block $U_{k}$. Let $x \in \mathbb{C}^{N}$ be the signal we wish to recover, where $N=N_{r}$. The question is, how many samples $m=m_{1}+\cdots+m_{r}$ do we require?

Suppose we assume that $x$ is $s$-sparse, where $s \leqslant \min _{k=1, \ldots, r}\left\{N_{k}-N_{k-1}\right\}$. Given no further information about the sparsity structure, it is necessary to take $m_{k} \gtrsim s \log (N)$ measurements in each block, giving $m \gtrsim r s \log (N)$ in total. However, suppose now that $x$ is known to be $s_{k}$-sparse within each level, that is $\left|\operatorname{supp}(x) \cap\left\{N_{k-1}+1, \ldots, N_{k}\right\}\right|=s_{k}$. Then we now require only $m_{k} \gtrsim s_{k} \log (N)$, and therefore $m \gtrsim s \log (N)$ total measurements. Thus, structured sparsity leads to a significant saving by a factor of $r$ in the total number of measurements required. Although a cosmetic example, we note in passing that the Fourier-wavelets matrix is approximately block diagonal with incoherent blocks, and that the number of levels $r$ in this case is proportional to the log of the signal size.

\section{Main theorems II: the infinite-dimensional case}

Finite-dimensional CS is suitable in many cases. However, there are some important problems where it can lead to significant problems, since the underlying problem is continuous/analogue. Discretization of the problem in order to produce a finite-dimensional, vector-space model can lead to substantial errors $[3,7,22$, 76], due to the phenomenon of model mismatch.

To address this issue, a theory of infinite-dimensional CS was introduced by two of the authors in [3], based on a novel approach to classical sampling known 
as generalized sampling $[\mathbf{1}, \mathbf{2}, \mathbf{4 - 6}, \mathbf{5 2}]$. We describe this theory next. Note that this infinite-dimensional CS model has also been advocated for and implemented in MRI by Guerquin-Kern, Häberlin, Pruessmann and Unser [45]. See also [53] for a practical implementation of infinite-dimensional CS in surface scattering.

5.1. Infinite-dimensional CS. Suppose that $\mathcal{H}$ is a separable Hilbert space over $\mathbb{C}$, and let $\left\{\psi_{j}\right\}_{j \in \mathbb{N}}$ be an orthonormal basis on $\mathcal{H}$ (the sampling basis). Let $\left\{\varphi_{j}\right\}_{j \in \mathbb{N}}$ be an orthonormal system in $\mathcal{H}$ (the sparsity system), and suppose that

$$
U=\left(u_{i j}\right)_{i, j \in \mathbb{N}}, \quad u_{i j}=\left\langle\varphi_{j}, \psi_{i}\right\rangle,
$$

is an infinite matrix. We may consider $U$ as an element of $\mathcal{B}\left(l^{2}(\mathbb{N})\right)$; the space of bounded operators on $l^{2}(\mathbb{N})$. As in the finite-dimensional case, $U$ is an isometry, and we may define its coherence $\mu(U) \in(0,1]$ analogously to (2.1). We want to recover $f=\sum_{j \in \mathbb{N}} x_{j} \varphi_{j} \in \mathcal{H}$ from a small number of the measurements $\hat{f}=\left\{\hat{f}_{j}\right\}_{j \in \mathbb{N}}$, where $\hat{f}_{j}=\left\langle f, \psi_{j}\right\rangle$. To do this, we introduce a second parameter $N \in \mathbb{N}$, and let $\Omega$ be a randomly chosen subset of indices $1, \ldots, N$ of size $m$. Unlike in finite dimensions, we now consider two cases. Suppose first that $P_{M}^{\perp} x=0$, that is $x$ has no tail. Then we solve

$$
\inf _{\eta \in l^{1}(\mathbb{N})}\|\eta\|_{l^{1}} \text { subject to }\left\|P_{\Omega} U P_{M} \eta-y\right\| \leqslant \delta,
$$

where $y=P_{\Omega} \hat{f}+z$ and $z \in \operatorname{ran}\left(P_{\Omega}\right)$ is a noise vector satisfying $\|z\| \leqslant \delta$, and $P_{\Omega}$ is the projection operator corresponding to the index set $\Omega$. In [3] it was proved that any solution to (5.2) recovers $f$ exactly up to an error determined by $\sigma_{s, M}(f)$, provided $N$ and $m$ satisfy the so-called weak balancing property with respect to $M$ and $s$ (see Definition 5.1, as well as Remark 5.4 for a discussion), and provided

$$
m \gtrsim \mu(U) \cdot N \cdot s \cdot\left(1+\log \left(\epsilon^{-1}\right)\right) \cdot \log \left(m^{-1} M N \sqrt{s}\right) .
$$

As in the finite-dimensional case, which turns out to be a corollary of this result, we find that $m$ is on the order of the sparsity $s$ whenever $\mu(U)$ is sufficiently small.

In practice, the condition $P_{M}^{\perp} x=0$ is unrealistic. In the more general case, $P_{M}^{\perp} x \neq 0$, we solve the following problem:

$$
\inf _{\eta \in l^{1}(\mathbb{N})}\|\eta\|_{l^{1}} \text { subject to }\left\|P_{\Omega} U \eta-y\right\| \leqslant \delta .
$$

In [3] it was shown that any solution of (5.4) recovers $f$ exactly up to an error determined by $\sigma_{s, M}(f)$, provided $N$ and $m$ satisfy the so-called strong balancing property with respect to $M$ and $s$ (see Definition 5.1), and provided 
a bound similar to (5.3) holds, where the $M$ is replaced by a slightly larger constant (we give the details in the next section in the more general setting of multilevel sampling). Note that (5.4) cannot be solved numerically, since it is infinite-dimensional. Therefore, in practice we replace (5.4) by

$$
\inf _{\eta \in l^{1}(\mathbb{N})}\|\eta\|_{l^{1}} \text { subject to }\left\|P_{\Omega} U P_{R} \eta-y\right\| \leqslant \delta,
$$

where $R$ is taken sufficiently large. See [3] for more information.

5.2. Main theorems. We first require the definition of the so-called balancing property [3]:

DEFINITION 5.1 (Balancing property). Let $U \in \mathcal{B}\left(l^{2}(\mathbb{N})\right)$ be an isometry. Then $N \in \mathbb{N}$ and $K \geqslant 1$ satisfy the weak balancing property with respect to $U, M \in \mathbb{N}$ and $s \in \mathbb{N}$ if

$$
\left\|P_{M} U^{*} P_{N} U P_{M}-P_{M}\right\|_{l^{\infty} \rightarrow l^{\infty}} \leqslant \frac{1}{8}\left(\log _{2}^{1 / 2}(4 \sqrt{s} K M)\right)^{-1},
$$

where $\|\cdot\|_{l^{\infty} \rightarrow l^{\infty}}$ is the norm on $\mathcal{B}\left(l^{\infty}(\mathbb{N})\right)$. We say that $N$ and $K$ satisfy the strong balancing property with respect to $U, M$ and $s$ if (5.6) holds, as well as

$$
\left\|P_{M}^{\perp} U^{*} P_{N} U P_{M}\right\|_{l^{\infty} \rightarrow l^{\infty}} \leqslant \frac{1}{8} .
$$

As in the previous section, we commence with the two-level case. Furthermore, to illustrate the differences between the weak/strong balancing property, we first consider the setting of (5.2):

THEOREM 5.2. Let $U \in \mathcal{B}\left(l^{2}(\mathbb{N})\right)$ be an isometry and $x \in l^{1}(\mathbb{N})$. Suppose that $\Omega=\Omega_{\mathbf{N}, \mathbf{m}}$ is a two-level sampling scheme, where $\mathbf{N}=\left(N_{1}, N_{2}\right)$ and $\mathbf{m}=\left(N_{1}, m_{2}\right)$. Let $(\mathbf{s}, \mathbf{M})$, where $\mathbf{M}=\left(M_{1}, M_{2}\right) \in \mathbb{N}^{2}, M_{1}<M_{2}$, and $\mathbf{s}=\left(M_{1}, s_{2}\right) \in \mathbb{N}^{2}$, be any pair such that the following holds:

(i) we have $\left\|P_{N_{1}}^{\perp} U P_{M_{1}}\right\| \leqslant \gamma / \sqrt{M_{1}}$ and $\gamma \leqslant s_{2} \sqrt{\mu_{N_{1}}}$ for some $\gamma \in(0,2 / 5]$;

(ii) the parameters $N=N_{2}, K=\left(N_{2}-N_{1}\right) / m_{2}$ satisfy the weak balancing property with respect to $U, M:=M_{2}$ and $s:=M_{1}+s_{2}$;

(iii) for $\epsilon \in\left(0, e^{-1}\right]$, let

$$
m_{2} \gtrsim\left(N-N_{1}\right) \cdot \log \left(\epsilon^{-1}\right) \cdot \mu_{N_{1}} \cdot s_{2} \cdot \log (K M \sqrt{s}) .
$$


Suppose that $P_{M_{2}}^{\perp} x=0$ and let $\xi \in l^{1}(\mathbb{N})$ be a minimizer of (5.2) with $\delta=\tilde{\delta} \sqrt{K^{-1}}$. Then, with probability exceeding $1-s \epsilon$, we have

$$
\|\xi-x\| \leqslant C \cdot\left(\tilde{\delta} \cdot(1+L \cdot \sqrt{s})+\sigma_{\mathbf{s}, \mathbf{M}}(f)\right),
$$

for some constant $C$, where $\sigma_{\mathbf{S}, \mathbf{M}}(f)$ is as in (3.1), and $L=1+\left(\sqrt{\log _{2}\left(6 \epsilon^{-1}\right)} /\right.$ $\left.\log _{2}(4 K M \sqrt{s})\right)$. If $m_{2}=N-N_{1}$ then this holds with probability 1 .

We next state a result for multilevel sampling in the more general setting of (5.4). For this, we require the following notation: $\tilde{M}=\min \{i \in \mathbb{N}$ : $\left.\max _{k \geqslant i}\left\|P_{N} U e_{k}\right\| \leqslant 1 /(32 K \sqrt{s})\right\}$, where $N, s$ and $K$ are as defined below.

THEOREM 5.3. Let $U \in \mathcal{B}\left(l^{2}(\mathbb{N})\right)$ be an isometry and $x \in l^{1}(\mathbb{N})$. Suppose that $\Omega=\Omega_{\mathbf{N}, \mathbf{m}}$ is a multilevel sampling scheme, where $\mathbf{N}=\left(N_{1}, \ldots, N_{r}\right) \in \mathbb{N}^{r}$ and $\mathbf{m}=\left(m_{1}, \ldots, m_{r}\right) \in \mathbb{N}^{r}$. Let $(\mathbf{s}, \mathbf{M})$, where $\mathbf{M}=\left(M_{1}, \ldots, M_{r}\right) \in \mathbb{N}^{r}$, $M_{1}<\cdots<M_{r}$, and $\mathbf{s}=\left(s_{1}, \ldots, s_{r}\right) \in \mathbb{N}^{r}$, be any pair such that the following holds:

(i) the parameters $N=N_{r}, K=\max _{k=1, \ldots, r}\left\{\left(N_{k}-N_{k-1}\right) / m_{k}\right\}$, satisfy the strong balancing property with respect to $U, M:=M_{r}$ and $s:=s_{1}+\cdots+s_{r}$;

(ii) for $\epsilon \in\left(0, e^{-1}\right]$ and $1 \leqslant k \leqslant r$,

$$
1 \gtrsim \frac{N_{k}-N_{k-1}}{m_{k}} \cdot \log \left(\epsilon^{-1}\right) \cdot\left(\sum_{l=1}^{r} \mu_{\mathbf{N}, \mathbf{M}}(k, l) \cdot s_{l}\right) \cdot \log (K \tilde{M} \sqrt{s}),
$$

(with $\mu_{\mathbf{N}, \mathbf{M}}(k, r)$ replaced by $\mu_{\mathbf{N}, \mathbf{M}}(k, \infty)$ ) and

$$
m_{k} \gtrsim \hat{m}_{k} \cdot \log \left(\epsilon^{-1}\right) \cdot \log (K \tilde{M} \sqrt{s}),
$$

where $\hat{m}_{k}$ satisfies (4.5).

Suppose that $\xi \in l^{1}(\mathbb{N})$ is a minimizer of (5.4) with $\delta=\tilde{\delta} \sqrt{K^{-1}}$. Then, with probability exceeding $1-s \epsilon$,

$$
\|\xi-x\| \leqslant C \cdot\left(\tilde{\delta} \cdot(1+L \cdot \sqrt{s})+\sigma_{\mathbf{s}, \mathbf{M}}(f)\right),
$$

for some constant $C$, where $\sigma_{\mathbf{s}, \mathbf{M}}(f)$ is as in (3.1), and $L=C \cdot(1+$ $\left.\left(\sqrt{\log _{2}\left(6 \epsilon^{-1}\right)} / \log _{2}(4 K M \sqrt{s})\right)\right)$. If $m_{k}=N_{k}-N_{k-1}$ for $1 \leqslant k \leqslant r$ then this holds with probability 1 . 
This theorem removes the condition in Theorem 5.2 that $x$ has zero tail. Note that the price to pay is the $\tilde{M}$ in the logarithmic term rather than $M(\tilde{M} \geqslant M$ because of the balancing property). Observe that $\tilde{M}$ is finite, and in the case of Fourier sampling with wavelets, we have that $\tilde{M}=\mathcal{O}(K N)$ (see Section 6). Note that Theorem 5.2 has a strong form analogous to Theorem 5.3 which removes the tail condition. The only difference is the requirement of the strong, as opposed to the weak, balancing property, and the replacement of $M$ by $\tilde{M}$ in the $\log$ factor. Similarly, Theorem 5.3 has a weak form involving a tail condition. For succinctness we do not state these.

REMARK 5.4. The balancing property is the main difference between the finiteand infinite-dimensional theorems. Its role is to ensure that the truncated matrix $P_{N} U P_{M}$ is close to an isometry. In reconstruction problems, the presence of an isometry ensures stability in the mapping between measurements and coefficients [1], which explains the need for a such a property in our theorems. As explained in [3], without the balancing property the lack of stability in this mapping leads to numerically useless reconstructions. Note that the balancing property is usually not satisfied for $N=M$, and in general one requires $N>M$ for it to hold. However, there is always a finite value of $N$ for which it is satisfied, since the infinite matrix $U$ is an isometry. For details we refer to [3]. We provide specific estimates in Section 6 for the required magnitude of $N$ in the case of Fourier sampling with wavelet sparsity.

5.3. The need for infinite-dimensional CS. As mentioned above, infinitedimensional CS is needed to avoid artefacts that are introduced when one applies finite-dimensional CS techniques to analogue problems. To illustrate this, we consider the problem of recovering a smooth phantom, that is a $C^{\infty}$ bivariate function, from its Fourier data. Note that this scenario arises in both electron microscopy and spectroscopy. In Figure 5, we compare finite-dimensional CS, based on solving (4.1) with $U=U_{\mathrm{dft}} V_{\mathrm{dwt}}^{-1}$ (discrete Fourier and wavelet transform respectively) with infinite-dimensional CS, which solves (5.5) with the Fourier basis $\left\{\psi_{j}\right\}_{j \in \mathbb{N}}$ and boundary wavelet basis $\left\{\varphi_{j}\right\}_{j \in \mathbb{N}}$. The test function in this case is $f(x, y)=\cos ^{2}(17 \pi x / 2) \cos ^{2}(17 \pi y / 2) \exp (-x-y)$. The improvement one gets is due to that fact that the error in infinite-dimensional case is dominated by the wavelet approximation error, whereas in the finite-dimensional case (due mismatch between the continuous Fourier samples and the discrete Fourier transform) the error is dominated by the Fourier approximation error. As is well known [64], wavelet approximation is superior to Fourier approximation and depends on the number of vanishing moments of the wavelet used (DB4 in this case). 


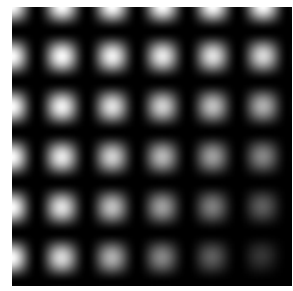

Original

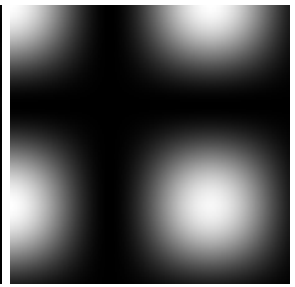

Original (zoom)

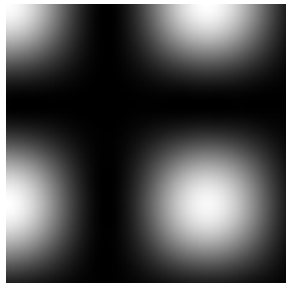

Inf.-dim. CS (zoom) Err $0.6 \%$

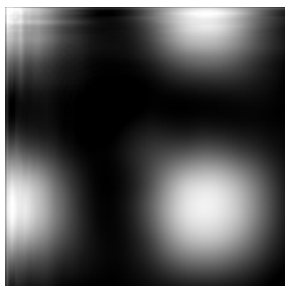

Fin.-dim. CS (zoom)

Err $12.7 \%$

Figure 5. Subsampling 6.15\%. Both reconstructions are based on identical sampling information.

\section{Recovery of wavelet coefficients from Fourier samples}

As noted, Fourier sampling with wavelet sparsity is an important reconstruction problem in CS, with numerous applications ranging from medical imaging to seismology and interferometry. Here we consider the Fourier sampling basis $\left\{\psi_{j}\right\}_{j \in \mathbb{N}}$ and wavelet reconstruction basis $\left\{\varphi_{j}\right\}_{j \in \mathbb{N}}$ (see Section 7.4.1 for a formal definition) with the infinite matrix $U$ as in (5.1). Its global incoherence properties are summarized as follows:

THEOREM 6.1. Let $U \in \mathcal{B}\left(l^{2}(\mathbb{N})\right)$ be the matrix from (7.107) corresponding to the Fourier-wavelets system described in Section 7.4. Then $\mu(U) \geqslant \omega$, where $\omega$ is the sampling density, whereas $\mu\left(P_{N}^{\perp} U\right), \mu\left(U P_{N}^{\perp}\right)=\mathcal{O}\left(N^{-1}\right)$.

Thus, Fourier sampling with wavelet sparsity is indeed globally coherent, yet asymptotically incoherent. This result holds for essentially any wavelet basis in one dimension (see [54] for the multidimensional case). To recover wavelet coefficients, we shall therefore seek to apply a multilevel sampling strategy, which raises the questions: how do we design this strategy, and how many measurements are required? If the levels $\mathbf{M}=\left(M_{1}, \ldots, M_{r}\right)$ correspond to the wavelet scales, and $\mathbf{s}=\left(s_{1}, \ldots, s_{r}\right)$ to the sparsities within them, then the best one could hope to achieve is that the number of measurements $m_{k}$ in the $k$ th sampling level is proportional to the sparsity $s_{k}$ in the corresponding sparsity level. Our main theorem below shows that multilevel sampling can achieve this, up to an exponentially localized factor and the usual log terms.

THEOREM 6.2. Consider an orthonormal basis of compactly supported wavelets with a multiresolution analysis (MRA). Let $\Phi$ and $\Psi$ denote the scaling function and mother wavelet respectively satisfying (7.100) with $\alpha \geqslant 1$. Suppose that $\Psi$ 
has $v \geqslant 1$ vanishing moments, that the Fourier sampling density $\omega$ satisfies (7.105) and that the wavelets $\left\{\varphi_{j}\right\}$ are ordered according to (7.102). Let $f=\sum_{j=1}^{\infty} x_{j} \varphi_{j}$. Suppose that $\mathbf{M}=\left(M_{1}, \ldots, M_{r}\right)$ corresponds to wavelet scales with $M_{k}=\mathcal{O}\left(2^{R_{k}}\right)$ with $R_{k} \in \mathbb{N}, R_{k+1}=a+R_{k}, a \geqslant 1, k=1, \ldots, r$ and $\mathbf{s}=\left(s_{1}, \ldots, s_{r}\right)$ corresponds to the sparsities within them. Let $\epsilon \in\left(0, e^{-1}\right]$ and let $\Omega=\Omega_{\mathrm{N}, \mathrm{m}}$ be a multilevel sampling scheme such that the following holds:

(i) The parameters $N=N_{r}, K=\max _{k=1, \ldots, r},\left\{\left(N_{k}-N_{k-1}\right) / m_{k}\right\}, M=M_{r}$, $s=s_{1}+\cdots+s_{r}$ satisfy $N \gtrsim M^{1+1 /(2 \alpha-1)} \cdot\left(\log _{2}(4 M K \sqrt{s})\right)^{1 /(2 \alpha-1)}$. Alternatively, if $\Phi$ and $\Psi$ satisfy the slightly stronger Fourier decay property (7.101), then $N \gtrsim M \cdot\left(\log _{2}(4 K M \sqrt{s})\right)^{1 /(4 \alpha-2)}$.

(ii) For each $k=1, \ldots, r-1, N_{k}=2^{R_{k}} \omega^{-1}$ and for each $k=1, \ldots, r$,

$$
\begin{aligned}
m_{k} \gtrsim & \log \left(\epsilon^{-1}\right) \cdot \log (\tilde{N}) \cdot \frac{N_{k}-N_{k-1}}{N_{k-1}} \\
& \times\left(\hat{s}_{k}+\sum_{l=1}^{k-2} s_{l} \cdot 2^{-(\alpha-1 / 2) A_{k, l}}+\sum_{l=k+2}^{r} s_{l} \cdot 2^{-v B_{k, l}}\right),
\end{aligned}
$$

where $A_{k, l}=R_{k-1}-R_{l}, B_{k, l}=R_{l-1}-R_{k}, \quad \tilde{N}=(K \sqrt{s})^{1+1 / v} N$ and $\hat{s}_{k}=\max \left\{s_{k-1}, s_{k}, s_{k+1}\right\}$ (see Remark 6.3).

Then, with probability exceeding $1-s \epsilon$, any minimizer $\xi \in l^{1}(\mathbb{N})$ of (5.4) with $\delta=\tilde{\delta} \sqrt{K^{-1}}$ satisfies

$$
\|\xi-x\| \leqslant C \cdot\left(\tilde{\delta} \cdot(1+L \cdot \sqrt{s})+\sigma_{\mathbf{s}, \mathbf{M}}(f)\right),
$$

for some constant $C$, where $\sigma_{\mathbf{s}, \mathbf{M}}(f)$ is as in (3.1), and $L=C \cdot(1+$ $\left.\left(\sqrt{\log _{2}\left(6 \epsilon^{-1}\right)} / \log _{2}(4 K M \sqrt{s})\right)\right)$. If $m_{k}=N_{k}-N_{k-1}$ for $1 \leqslant k \leqslant r$ then this holds with probability 1.

REMARK 6.3. To avoid cluttered notation we have abused notation slightly in (ii) of Theorem 6.2. In particular, we interpret $s_{0}=0,\left(N_{k}-N_{k-1}\right) / N_{k-1}=N_{1}$ for $k=1$, and $\sum_{l=1}^{k-2} s_{l} \cdot 2^{-(\alpha-1 / 2) A_{k, l}}=0$ when $k \leqslant 2$.

Given that one can never solve (5.4) exactly, but rather the truncated version (5.5), the following proposition provides the guidance on how the truncation needs to be carried out in order to obtain the same error bound as in Theorem 6.2. 
Proposition 6.4. Given the setup in Theorem 6.2 with $\xi \in l^{1}(\mathbb{N})$ being a minimizer of (5.4), thus satisfying the error bound (6.2). Let $J$ be the number of wavelets up to dilation

$$
R=\log _{2}\left(2 \pi \omega N\left(\frac{\left\|P_{K}^{\perp} x\right\|_{2} \cdot C_{\Psi}}{\delta}\right)^{1 / v}\right),
$$

where $C_{\Psi}$ is a constant depending only on the underlying wavelet $\Psi$. If one instead solves

$$
\min _{\eta \in \in \mathbb{C}^{J}}\|\eta\|_{l^{1}} \text { subject to }\left\|P_{\Omega} U P_{J} \eta-y\right\| \leqslant 2 \delta,
$$

then any minimizer $\tilde{\xi}$ of (6.3) satisfies the same error bound (6.2) as $\xi$ with a potentially different constant $C$.

Theorem 6.2 provides the first comprehensive explanation for the observed success of CS in applications based on the Fourier-wavelets model. The key estimate (6.1) shows that $m_{k}$ need only scale as a linear combination of the local sparsities $s_{l}, 1 \leqslant l \leqslant r$, and critically, the dependence of the sparsities $s_{l}$ for $l \neq k$ is exponentially diminishing in $|k-l|$. Note that the presence of the offdiagonal terms is due to the previously discussed phenomenon of interference, which occurs since the Fourier-wavelets system is not exactly block diagonal. Nonetheless, the system is nearly block diagonal, and this results in the near optimality seen in (6.1).

Observe that (6.1) is in agreement with the flip test: if the local sparsities $s_{k}$ change, then the subsampling factors $m_{k}$ must also change to ensure the same quality reconstruction. Having said that, it is straightforward to deduce from (6.1) the following global sparsity bound:

$$
m \gtrsim s \cdot \log \left(\epsilon^{-1}\right) \cdot \log (\tilde{N}),
$$

where $m=m_{1}+\cdots+m_{r}$ is the total number of measurements and $s=s_{1}+\cdots+s_{r}$ is the total sparsity. Note in particular the optimal exponent in the log factor.

REMARK 6.5. The Fourier/wavelets recovery problem was studied by Candès and Romberg in [17]. Their result shows that if, in an ideal setting, an image can be first separated into separate wavelet subbands before sampling, then it can be recovered using approximately $s_{k}$ measurements (up to a log factor) in each sampling band. Unfortunately, such separation into separate wavelet subbands before sampling is infeasible in most practical situations. Theorem 6.2 improves on this result by removing this substantial restriction, with the sole penalty being the slightly worse bound (6.1). 


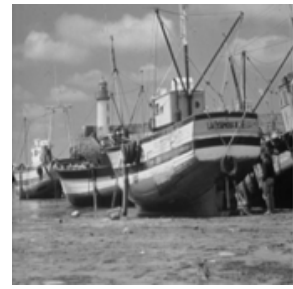

Original image

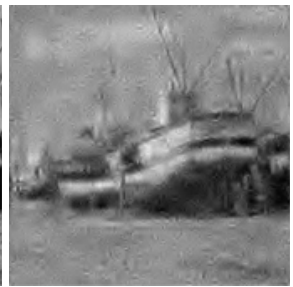

Random Bernoulli Err $=15.7 \%$

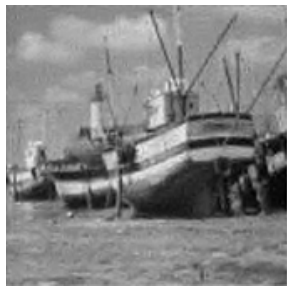

Multilevel Hadamard Err $=9.6 \%$

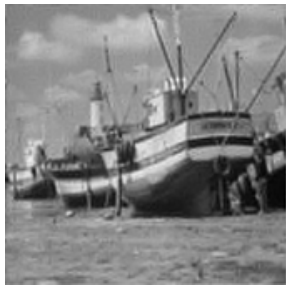

Multilevel Fourier Err $8.7 \%$

Figure 6. $12.5 \%$ subsampling at $256 \times 256$ resolution using DB4 wavelets and various different measurements.

Note also that a recovery result for bivariate Haar wavelets, as well as the related technique of TV minimization, was given in [55]. Similarly [10] analyzes block sampling strategies with application to MRI. However, these results are based on sparsity, and therefore they do not explain the conclusions of the flip test regarding how the sampling strategy will depend on the signal structure.

6.1. Universality and RIP or structure? Theorem 6.2 explains the success of CS when one is constrained to acquire Fourier measurements. Yet, due primarily to the their high global coherence with wavelets, Fourier measurements are often viewed as suboptimal for CS. If one had complete freedom to choose the measurements, and no physical constraints (such as are always present in MRI, for example), then standard CS intuition would suggest random Gaussian or Bernoulli measurements, since they are universal and satisfy the RIP.

However, in reality such measurements are actually highly suboptimal in the presence of structured sparsity. This is demonstrated in Figure 6, where an image is recovered from $m=8192$ measurements taken either as random Bernoulli or multilevel Hadamard or Fourier type. As is evident, the latter gives an error that is almost 50\% smaller. The reason for this improvement is that whilst Fourier or Hadamard measurements are highly coherent with wavelets, they are asymptotically incoherent, and, as explained in our theoretical results, this can be exploited through multilevel random subsampling to recover the structured (that is asymptotica) sparsity of wavelet coefficients. Random Gaussian/Bernoulli measurements on the other hand do take advantage of this structure since, in satisfying an RIP, they are guaranteed to recover all sparse vectors equally well.

This observation is an important consequence of our framework. Specifically, whenever structured sparsity is present (such is the case in the majority of 
imaging applications, for example) there are substantial improvements to be gained by designing the measurements according to not just the sparsity, but also the additional structure. For a more comprehensive discussion see [73], as well as $[19,83]$.

\section{Proofs}

The proofs rely on some key propositions from which one can deduce the main theorems. The main work is to prove these proposition, and that will be done subsequently.

\subsection{Key results.}

Proposition 7.1. Let $U \in \mathcal{B}\left(l^{2}(\mathbb{N})\right)$ and suppose that $\Delta$ and $\Omega=\Omega_{1} \cup \cdots \cup \Omega_{r}$ (where the union is disjoint) are subsets of $\mathbb{N}$. Let $x_{0} \in \mathcal{H}$ and $z \in \operatorname{ran}\left(P_{\Omega} U\right)$ be such that $\|z\| \leqslant \delta$ for $\delta \geqslant 0$. Let $M \in \mathbb{N}$ and $y=P_{\Omega} U x_{0}+z$ and $y_{M}=P_{\Omega} U P_{M} x_{0}+z$. Suppose that $\xi \in \mathcal{H}$ and $\xi_{M} \in \mathcal{H}$ satisfy

$$
\begin{gathered}
\|\xi\|_{l^{1}}=\inf _{\eta \in \mathcal{H}}\left\{\|\eta\|_{l^{1}}:\left\|P_{\Omega} U \eta-y\right\| \leqslant \delta\right\}, \\
\left\|\xi_{M}\right\|_{l^{1}}=\inf _{\eta \in \mathbb{C}^{M}}\left\{\|\eta\|_{l^{1}}:\left\|P_{\Omega} U P_{M} \eta-y_{M}\right\| \leqslant \delta\right\} .
\end{gathered}
$$

If there exists a vector $\rho=U^{*} P_{\Omega} w$ such that

(i) $\left\|P_{\Delta} U^{*}\left(q_{1}^{-1} P_{\Omega_{1}} \oplus \cdots \oplus q_{r}^{-1} P_{\Omega_{r}}\right) U P_{\Delta}-I_{\Delta}\right\| \leqslant \frac{1}{4}$

(ii) $\max _{i \in \Delta^{c}}\left\|\left(q_{1}^{-1 / 2} P_{\Omega_{1}} \oplus \cdots \oplus q_{r}^{-1 / 2} P_{\Omega_{r}}\right) U e_{i}\right\| \leqslant \sqrt{\frac{5}{4}}$

(iii) $\left\|P_{\Delta} \rho-\operatorname{sgn}\left(P_{\Delta} x_{0}\right)\right\| \leqslant q / 8$

(iv) $\left\|P_{\Delta}^{\perp} \rho\right\|_{l_{\infty}} \leqslant \frac{1}{2}$

(v) $\|w\| \leqslant L \cdot \sqrt{|\Delta|}$

for some $L>0$ and $0<q_{k} \leqslant 1, k=1, \ldots, r$, then we have that

$$
\left\|\xi-x_{0}\right\| \leqslant C \cdot\left(\delta \cdot\left(\frac{1}{\sqrt{q}}+L \sqrt{s}\right)+\left\|P_{\Delta}^{\perp} x_{0}\right\|_{l^{1}}\right),
$$

for some constant $C$, where $s=|\Delta|$ and $q=\min \left\{q_{k}\right\}_{k=1}^{r}$. Also, if (ii) is replaced by

$$
\max _{i \in\{1, \ldots, M\} \cap \Delta^{c}}\left\|\left(q_{1}^{-1 / 2} P_{\Omega_{1}} \oplus \cdots \oplus q_{r}^{-1 / 2} P_{\Omega_{r}}\right) U e_{i}\right\| \leqslant \sqrt{\frac{5}{4}}
$$


and (iv) is replaced by $\left\|P_{M} P_{\Delta}^{\perp} \rho\right\|_{l^{\infty}} \leqslant \frac{1}{2}$ then

$$
\left\|\xi_{M}-x_{0}\right\| \leqslant C \cdot\left(\delta \cdot\left(\frac{1}{\sqrt{q}}+L \sqrt{s}\right)+\left\|P_{M} P_{\Delta}^{\perp} x_{0}\right\|_{l^{1}}\right) .
$$

Proof. First observe that (i) implies that

$$
\left(\left.P_{\Delta} U^{*}\left(q_{1}^{-1} P_{\Omega_{1}} \oplus \cdots \oplus q_{r}^{-1} P_{\Omega_{r}}\right) U P_{\Delta}\right|_{P_{\Delta}(\mathcal{H})}\right)^{-1}
$$

exists and

$$
\left\|\left(\left.P_{\Delta} U^{*}\left(q_{1}^{-1} P_{\Omega_{1}} \oplus \cdots \oplus q_{r}^{-1} P_{\Omega_{r}}\right) U P_{\Delta}\right|_{P_{\Delta}(\mathcal{H})}\right)^{-1}\right\| \leqslant \frac{4}{3} .
$$

Also, (i) implies that

$$
\begin{aligned}
& \left\|\left(q_{1}^{-1 / 2} P_{\Omega_{1}} \oplus \cdots \oplus q_{r}^{-1 / 2} P_{\Omega_{r}}\right) U P_{\Delta}\right\|^{2} \\
& \quad=\left\|P_{\Delta} U^{*}\left(q_{1}^{-1} P_{\Omega_{1}} \oplus \cdots \oplus q_{r}^{-1} P_{\Omega_{r}}\right) U P_{\Delta}\right\| \leqslant \frac{5}{4},
\end{aligned}
$$

and

$$
\begin{aligned}
& \left\|P_{\Delta} U^{*}\left(q_{1}^{-1} P_{\Omega_{1}} \oplus \cdots \oplus q_{r}^{-1} P_{\Omega_{r}}\right)\right\|^{2}=\left\|\left(q_{1}^{-1} P_{\Omega_{1}} \oplus \cdots \oplus q_{r}^{-1} P_{\Omega_{r}}\right) U P_{\Delta}\right\|^{2} \\
& \quad=\sup _{\|\eta\|=1}\left\|\left(q_{1}^{-1} P_{\Omega_{1}} \oplus \cdots \oplus q_{r}^{-1} P_{\Omega_{r}}\right) U P_{\Delta} \eta\right\|^{2} \\
& \quad=\sup _{\|\eta\|=1} \sum_{k=1}^{r}\left\|q_{k}^{-1} P_{\Omega_{k}} U P_{\Delta} \eta\right\|^{2} \leqslant \frac{1}{q} \sup _{\|\eta\|=1} \sum_{k=1}^{r} q_{k}^{-1}\left\|P_{\Omega_{k}} U P_{\Delta} \eta\right\|^{2} \\
& \quad=\frac{1}{q} \sup _{\|\eta\|=1}\left\langle P_{\Delta} U^{*}\left(\sum_{k=1}^{r} q_{k}^{-1} P_{\Omega_{k}}\right) U P_{\Delta} \eta, \eta\right\rangle \\
& \quad \leqslant \frac{1}{q}\left\|P_{\Delta} U^{*}\left(q_{1}^{-1} P_{\Omega_{1}} \oplus \cdots \oplus q_{r}^{-1} P_{\Omega_{r}}\right) U P_{\Delta}\right\|
\end{aligned}
$$

where $1 / q=\max _{1 \leqslant k \leqslant r}\left\{1 / q_{k}\right\}$. Thus, (7.5) and (7.6) imply

$$
\left\|P_{\Delta} U^{*}\left(q_{1}^{-1} P_{\Omega_{1}} \oplus \cdots \oplus q_{r}^{-1} P_{\Omega_{r}}\right)\right\| \leqslant \sqrt{\frac{5}{4 q}} .
$$

Suppose that there exists a vector $\rho$, constructed with $y_{0}=P_{\Delta} x_{0}$, satisfying (iii)-(v). Let $\xi$ be a solution to (7.1) and let $h=\xi-x_{0}$. Let

$$
A_{\Delta}=\left.P_{\Delta} U^{*}\left(q_{1}^{-1} P_{\Omega_{1}} \oplus \cdots \oplus q_{r}^{-1} P_{\Omega_{r}}\right) U P_{\Delta}\right|_{P_{\Delta}(\mathcal{H})} .
$$


Then, it follows from (ii) and observations (7.4), (7.5), (7.7) that

$$
\begin{aligned}
\left\|P_{\Delta} h\right\|= & \left\|A_{\Delta}^{-1} A_{\Delta} P_{\Delta} h\right\| \\
\leqslant & \left\|A_{\Delta}^{-1}\right\|\left\|P_{\Delta} U^{*}\left(q_{1}^{-1} P_{\Omega_{1}} \oplus \cdots \oplus q_{r}^{-1} P_{\Omega_{r}}\right) U\left(I-P_{\Delta}^{\perp}\right) h\right\| \\
\leqslant & \frac{4}{3}\left\|P_{\Delta} U^{*}\left(q_{1}^{-1} P_{\Omega_{1}} \oplus \cdots \oplus q_{r}^{-1} P_{\Omega_{r}}\right)\right\|\left\|P_{\Omega} U h\right\| \\
& +\frac{4}{3} \max _{i \in \Delta^{c}}\left\|P_{\Delta} U^{*}\left(q_{1}^{-1} P_{\Omega_{1}} \oplus \cdots \oplus q_{r}^{-1} P_{\Omega_{r}}\right) U e_{i}\right\|\left\|P_{\Delta}^{\perp} h\right\|_{l^{1}} \\
\leqslant & \frac{4}{3}\left\|P_{\Delta} U^{*}\left(q_{1}^{-1} P_{\Omega_{1}} \oplus \cdots \oplus q_{r}^{-1} P_{\Omega_{r}}\right)\right\|\left\|P_{\Omega} U h\right\| \\
& +\frac{4}{3}\left\|P_{\Delta} U^{*}\left(q_{1}^{-1 / 2} P_{\Omega_{1}} \oplus \cdots \oplus q_{r}^{-1 / 2}\right)\right\| \\
& \times \max _{i \in \Delta^{c}}\left\|\left(q_{1}^{-1 / 2} P_{\Omega_{1}} \oplus \cdots \oplus q_{r}^{-1 / 2} P_{\Omega_{r}}\right) U e_{i}\right\|\left\|P_{\Delta}^{\perp} h\right\|_{l^{1}} \\
\leqslant & \frac{4 \sqrt{5}}{3 \sqrt{q}} \delta+\frac{5}{3}\left\|P_{\Delta}^{\perp} h\right\|_{l^{1}},
\end{aligned}
$$

where in the final step we use $\left\|P_{\Omega} U h\right\| \leqslant\left\|P_{\Omega} U \zeta-y\right\|+\|z\| \leqslant 2 \delta$. We now obtain a bound for $\left\|P_{\Delta}^{\perp} h\right\|_{l^{1}}$. First note that

$$
\begin{aligned}
\left\|h+x_{0}\right\|_{l^{1}} & =\left\|P_{\Delta} h+P_{\Delta} x_{0}\right\|_{l^{1}}+\left\|P_{\Delta}^{\perp}\left(h+x_{0}\right)\right\|_{l^{1}} \\
& \geqslant \operatorname{Re}\left\langle P_{\Delta} h, \operatorname{sgn}\left(P_{\Delta} x_{0}\right)\right\rangle+\left\|P_{\Delta} x_{0}\right\|_{l^{1}}+\left\|P_{\Delta}^{\perp} h\right\|_{l^{1}}-\left\|P_{\Delta}^{\perp} x_{0}\right\|_{l^{1}} \\
& \geqslant \operatorname{Re}\left\langle P_{\Delta} h, \operatorname{sgn}\left(P_{\Delta} x_{0}\right)\right\rangle+\left\|x_{0}\right\|_{l^{1}}+\left\|P_{\Delta}^{\perp} h\right\|_{l^{1}}-2\left\|P_{\Delta}^{\perp} x_{0}\right\|_{l^{1}} .
\end{aligned}
$$

Since $\left\|x_{0}\right\|_{l^{1}} \geqslant\left\|h+x_{0}\right\|_{l^{1}}$, we have that

$$
\left\|P_{\Delta}^{\perp} h\right\|_{l^{1}} \leqslant\left|\left\langle P_{\Delta} h, \operatorname{sgn}\left(P_{\Delta} x_{0}\right)\right\rangle\right|+2\left\|P_{\Delta}^{\perp} x_{0}\right\|_{l^{1}} .
$$

We use this equation later on in the proof, but before we do that observe that some basic adding and subtracting yields

$$
\begin{aligned}
\left|\left\langle P_{\Delta} h, \operatorname{sgn}\left(x_{0}\right)\right\rangle\right| \\
\quad \leqslant\left|\left\langle P_{\Delta} h, \operatorname{sgn}\left(P_{\Delta} x_{0}\right)-P_{\Delta} \rho\right\rangle\right|+|\langle h, \rho\rangle|+\left|\left\langle P_{\Delta}^{\perp} h, P_{\Delta}^{\perp} \rho\right\rangle\right| \\
\quad \leqslant\left\|P_{\Delta} h\right\|\left\|\operatorname{sgn}\left(P_{\Delta} x_{0}\right)-P_{\Delta} \rho\right\|+\left|\left\langle P_{\Omega} U h, w\right\rangle\right|+\left\|P_{\Delta}^{\perp} h\right\|_{l^{1}}\left\|P_{\Delta}^{\perp} \rho\right\|_{l^{\infty}} \\
\quad \leqslant \frac{q}{8}\left\|P_{\Delta} h\right\|+2 L \delta \sqrt{s}+\frac{1}{2}\left\|P_{\Delta}^{\perp} h\right\|_{l^{1}} \\
\quad \leqslant \frac{\sqrt{5 q}}{6} \delta+\frac{5 q}{24}\left\|P_{\Delta}^{\perp} h\right\|_{l^{1}}+2 L \delta \sqrt{s}+\frac{1}{2}\left\|P_{\Delta}^{\perp} h\right\|_{l^{1}}
\end{aligned}
$$

where the last inequality utilizes (7.8) and the penultimate inequality follows from properties (iii), (iv) and (v) of the dual vector $\rho$. Combining this with (7.10) and 
the fact that $q \leqslant 1$ gives that

$$
\left\|P_{\Delta}^{\perp} h\right\|_{l^{1}} \leqslant \delta\left(\frac{4 \sqrt{5 q}}{3}+8 L \sqrt{s}\right)+8\left\|P_{\Delta}^{\perp} x_{0}\right\|_{l^{1}} .
$$

Thus, (7.8) and (7.12) yields:

$$
\begin{aligned}
\|h\| \leqslant\left\|P_{\Delta} h\right\|+\left\|P_{\Delta}^{\perp} h\right\| & \leqslant \frac{8}{3}\left\|P_{\Delta}^{\perp} h\right\|_{l^{1}}+\frac{4 \sqrt{5}}{3 \sqrt{q}} \delta \\
& \leqslant\left(8 \sqrt{q}+22 L \sqrt{s}+\frac{3}{\sqrt{q}}\right) \cdot \delta+22\left\|P_{\Delta}^{\perp} x_{0}\right\|_{l^{1}} .
\end{aligned}
$$

The proof of the second part of this proposition follows the proof as outlined above and we omit the details.

The next two propositions give sufficient conditions for Proposition 7.1 to be true. But before we state them we need to define the following.

Definition 7.2. Let $U$ be an isometry of either $\mathbb{C}^{N \times N}$ or $\mathcal{B}\left(l^{2}(\mathbb{N})\right)$. For $\mathbf{N}=\left(N_{1}, \ldots, N_{r}\right) \in \mathbb{N}^{r}, \mathbf{M}=\left(M_{1}, \ldots, M_{r}\right) \in \mathbb{N}^{r}$ with $1 \leqslant N_{1}<\cdots<N_{r}$ and $1 \leqslant M_{1}<\cdots<M_{r}, \mathbf{s}=\left(s_{1}, \ldots, s_{r}\right) \in \mathbb{N}^{r}$ and $1 \leqslant k \leqslant r$, let

$$
\kappa_{\mathbf{N}, \mathbf{M}}(k, l)=\max _{\eta \in \Theta}\left\|P_{N_{k}}^{N_{k-1}} U P_{M_{l}}^{M_{l-1}} \eta\right\|_{l^{\infty}} \cdot \sqrt{\mu\left(P_{N_{k}}^{N_{k-1}} U\right)} .
$$

where

$\Theta=\left\{\eta:\|\eta\|_{l^{\infty}} \leqslant 1,\left|\operatorname{supp}\left(P_{M_{l}}^{M_{l-1}} \eta\right)\right|=s_{l}, l=1, \ldots, r-1,\left|\operatorname{supp}\left(P_{M_{r-1}}^{\perp} \eta\right)\right|=s_{r},\right\}$, and $N_{0}=M_{0}=0$. We also define

$$
\kappa_{\mathbf{N}, \mathbf{M}}(k, \infty)=\max _{\eta \in \Theta}\left\|P_{N_{k}}^{N_{k-1}} U P_{M_{r-1}}^{\perp} \eta\right\|_{l^{\infty}} \cdot \sqrt{\mu\left(P_{N_{k}}^{N_{k-1}} U\right)} .
$$

Proposition 7.3. Let $U \in \mathcal{B}\left(l^{2}(\mathbb{N})\right)$ be an isometry and $x \in l^{1}(\mathbb{N})$. Suppose that $\Omega=\Omega_{\mathbf{N}, \mathbf{m}}$ is a multilevel sampling scheme, where $\mathbf{N}=\left(N_{1}, \ldots, N_{r}\right) \in \mathbb{N}^{r}$ and $\mathbf{m}=\left(m_{1}, \ldots, m_{r}\right) \in \mathbb{N}^{r}$. Let $(\mathbf{s}, \mathbf{M})$, where $\mathbf{M}=\left(M_{1}, \ldots, M_{r}\right) \in \mathbb{N}^{r}$, $M_{1}<\cdots<M_{r}$, and $\mathbf{s}=\left(s_{1}, \ldots, s_{r}\right) \in \mathbb{N}^{r}$, be any pair such that the following holds:

(i) The parameters $N:=N_{r}$, and $K:=\max _{k=1, \ldots, r}\left(N_{k}-N_{k-1}\right) / m_{k}$, satisfy the weak balancing property with respect to $U, M:=M_{r}$ and $s:=s_{1}+\cdots+s_{r}$; 
(ii) for $\epsilon>0$ and $1 \leqslant k \leqslant r$,

$$
1 \gtrsim\left(\log \left(s \epsilon^{-1}\right)+1\right) \cdot \frac{N_{k}-N_{k-1}}{m_{k}} \cdot\left(\sum_{l=1}^{r} \kappa_{\mathbf{N}, \mathbf{M}}(k, l)\right) \cdot \log (K M \sqrt{s}),
$$

(iii)

$$
m_{k} \gtrsim\left(\log \left(s \epsilon^{-1}\right)+1\right) \cdot \hat{m}_{k} \cdot \log (K M \sqrt{s}),
$$

where $\hat{m}_{k}$ satisfies

$$
1 \gtrsim \sum_{k=1}^{r}\left(\frac{N_{k}-N_{k-1}}{\hat{m}_{k}}-1\right) \cdot \mu_{\mathbf{N}, \mathbf{M}}(k, l) \cdot \tilde{s}_{k}, \quad \forall l=1, \ldots, r,
$$

where $\tilde{s}_{1}+\cdots+\tilde{s}_{r} \leqslant s_{1}+\cdots+s_{r}, \tilde{s}_{k} \leqslant S_{k}\left(s_{1}, \ldots, s_{r}\right)$ and $S_{k}$ is defined in (4.4).

Then (i)-(v) in Proposition 7.1 follow with probability exceeding $1-\epsilon$, with (ii) replaced by

$$
\max _{i \in\{1, \ldots, M\} \cap \Delta^{c}}\left\|\left(q_{1}^{-1 / 2} P_{\Omega_{1}} \oplus \cdots \oplus q_{r}^{-1 / 2} P_{\Omega_{r}}\right) U e_{i}\right\| \leqslant \sqrt{\frac{5}{4}},
$$

(iv) replaced by $\left\|P_{M} P_{\Delta}^{\perp} \rho\right\|_{l^{\infty}} \leqslant \frac{1}{2}$ and $L$ in (v) is given by

$$
L=C \cdot \sqrt{K} \cdot\left(1+\frac{\sqrt{\log _{2}\left(6 \epsilon^{-1}\right)}}{\log _{2}(4 K M \sqrt{s})}\right) .
$$

If $m_{k}=N_{k}-N_{k-1}$ for all $1 \leqslant k \leqslant r$ then (i)-(v) follow with probability one (with the alterations suggested above).

Proposition 7.4. Let $U \in \mathcal{B}\left(l^{2}(\mathbb{N})\right)$ be an isometry and $x \in l^{1}(\mathbb{N})$. Suppose that $\Omega=\Omega_{\mathbf{N}, \mathbf{m}}$ is a multilevel sampling scheme, where $\mathbf{N}=\left(N_{1}, \ldots, N_{r}\right) \in \mathbb{N}^{r}$ and $\mathbf{m}=\left(m_{1}, \ldots, m_{r}\right) \in \mathbb{N}^{r}$. Let $(\mathbf{s}, \mathbf{M})$, where $\mathbf{M}=\left(M_{1}, \ldots, M_{r}\right) \in \mathbb{N}^{r}$, $M_{1}<\cdots<M_{r}$, and $\mathbf{s}=\left(s_{1}, \ldots, s_{r}\right) \in \mathbb{N}^{r}$, be any pair such that the following holds:

(i) The parameters $N$ and $K$ (as in Proposition 7.3) satisfy the strong balancing property with respect to $U, M=M_{r}$ and $s:=s_{1}+\cdots+s_{r}$; 
(ii) for $\epsilon>0$ and $1 \leqslant k \leqslant r$,

$$
\begin{aligned}
1 \gtrsim & \left(\log \left(s \epsilon^{-1}\right)+1\right) \cdot \frac{N_{k}-N_{k-1}}{m_{k}} \\
& \times\left(\kappa_{\mathbf{N}, \mathbf{M}}(k, \infty)+\sum_{l=1}^{r-1} \kappa_{\mathbf{N}, \mathbf{M}}(k, l)\right) \cdot \log (K \tilde{M} \sqrt{s}),
\end{aligned}
$$

(iii)

$$
m_{k} \gtrsim\left(\log \left(s \epsilon^{-1}\right)+1\right) \cdot \hat{m}_{k} \cdot \log (K \tilde{M} \sqrt{s})
$$

where $\tilde{M}=\min \left\{i \in \mathbb{N}:\left\|\max _{j \geqslant i} P_{N} U P_{\{j\}}\right\| \leqslant 1 /(K 32 \sqrt{s})\right\}$, and $\hat{m}_{k}$ is as in Proposition 7.3.

Then (i)-(v) in Proposition 7.1 follow with probability exceeding $1-\epsilon$ with $L$ as in (7.17). If $m_{k}=N_{k}-N_{k-1}$ for all $1 \leqslant k \leqslant r$ then (i)-(v) follow with probability one.

LEMMA 7.5 (Bounds for $\left.\kappa_{\mathbf{N}, \mathbf{M}}(k, l)\right)$. For $k, l=1, \ldots, r$

$$
\kappa_{\mathbf{N}, \mathbf{M}}(k, l) \leqslant \min \left\{\mu_{\mathbf{N}, \mathbf{M}}(k, l) \cdot s_{l}, \sqrt{s_{l} \cdot \mu\left(P_{N_{k}}^{N_{k-1}} U\right)} \cdot\left\|P_{N_{k}}^{N_{k-1}} U P_{M_{l}}^{M_{l-1}}\right\|\right\} .
$$

Also, for $k=1, \ldots, r$

$$
\kappa_{\mathbf{N}, \mathbf{M}}(k, \infty) \leqslant \min \left\{\mu_{\mathbf{N}, \mathbf{M}}(k, \infty) \cdot s_{r}, \sqrt{s_{r} \cdot \mu\left(P_{N_{k}}^{N_{k-1}} U\right)} \cdot\left\|P_{N_{k}}^{N_{k-1}} U P_{M_{r-1}}^{\perp}\right\|\right\} .
$$

Proof. For $k, l=1, \ldots, r$

$$
\begin{aligned}
\kappa_{\mathbf{N}, \mathbf{M}}(k, l) & =\max _{\eta \in \Theta}\left\|P_{N_{k}}^{N_{k-1}} U P_{M_{l}}^{M_{l-1}} \eta\right\|_{l \infty} \cdot \sqrt{\mu\left(P_{N_{k}}^{N_{k-1}} U\right)} \\
& =\max _{\eta \in \Theta} \max _{N_{k-1}<i \leqslant N_{k}}\left|\sum_{M_{l-1}<j \leqslant M_{l}} \eta_{j} u_{i j}\right| \cdot \sqrt{\mu\left(P_{N_{k}}^{N_{k-1}} U\right)} \\
& \leqslant s_{l} \cdot \sqrt{\mu\left(P_{N_{k}}^{N_{k-1}} U P_{M_{l}}^{M_{l-1}}\right)} \cdot \sqrt{\mu\left(P_{N_{k}}^{N_{k-1}} U\right)} \leqslant s_{l} \cdot \mu_{\mathbf{N}, \mathbf{M}}(k, l)
\end{aligned}
$$


since $\left|u_{i j}\right| \leqslant 1$, and similarly,

$$
\begin{aligned}
\kappa_{\mathbf{N}, \mathbf{M}}(k, \infty) & =\max _{\eta \in \Theta}\left\|P_{N_{k}}^{N_{k-1}} U P_{M_{r-1}}^{\perp} \eta\right\|_{l^{\infty}} \cdot \sqrt{\mu\left(P_{N_{k}}^{N_{k-1}} U\right)} \\
& =\max _{\eta \in \Theta} \max _{N_{k-1}<i \leqslant N_{k}}\left|\sum_{M_{r-1}<j} \eta_{j} u_{i j}\right| \cdot \sqrt{\mu\left(P_{N_{k}}^{N_{k-1}} U\right)} \leqslant s_{r} \cdot \mu_{\mathbf{N}, \mathbf{M}}(k, \infty) .
\end{aligned}
$$

Finally, it is straightforward to show that for $k, l=1, \ldots, r$,

$$
\kappa_{\mathbf{N}, \mathbf{M}}(k, l) \leqslant \sqrt{s_{l}} \cdot\left\|P_{N_{k}}^{N_{k-1}} U P_{M_{l}}^{M_{l-1}}\right\| \sqrt{\mu\left(P_{N_{k}}^{N_{k-1}} U\right)}
$$

and

$$
\kappa_{\mathbf{N}, \mathbf{M}}(k, \infty) \leqslant \sqrt{s_{r}} \cdot\left\|P_{N_{k}}^{N_{k-1}} U P_{M_{r-1}}^{\perp}\right\| \sqrt{\mu\left(P_{N_{k}}^{N_{k-1}} U\right)} .
$$

We are now ready to prove the main theorems.

Proof of Theorems 4.1 and 5.2. It is clear that Theorem 4.1 follows from Theorem 5.2, thus it remains to prove the latter. We apply Proposition 7.3 to a two-level sampling scheme $\Omega=\Omega_{\mathbf{N}, \mathbf{m}}$, where $\mathbf{N}=\left(N_{1}, N_{2}\right)$ and $\mathbf{m}=\left(m_{1}\right.$, $m_{2}$ ) with $m_{1}=N_{1}$ and $m_{2}=m$. Also, consider $(\mathbf{s}, \mathbf{M})$, where $\mathbf{s}=\left(M_{1}, s_{2}\right)$, $\mathbf{M}=\left(M_{1}, M_{2}\right)$. Thus, if $N_{1}, N_{2}, m_{1}, m_{2} \in \mathbb{N}$ are such that

$$
N=N_{2}, \quad K=\max \left\{\frac{N_{2}-N_{1}}{m_{2}}, \frac{N_{1}}{m_{1}}\right\}
$$

satisfy the weak balancing property with respect to $U, M=M_{2}$ and $s=M_{1}+s_{2}$, we have that (i)-(v) in Proposition 7.1 follow with probability exceeding $1-s \epsilon$, with (ii) replaced by

$$
\max _{i \in\{1, \ldots, M\} \cap \Delta^{c}}\left\|\left(P_{N_{1}} \oplus \frac{N_{2}-N_{1}}{m_{2}} P_{\Omega_{2}}\right) U e_{i}\right\| \leqslant \sqrt{\frac{5}{4}},
$$

(iv) replaced by $\left\|P_{M} P_{\Delta}^{\perp} \rho\right\|_{l^{\infty}} \leqslant \frac{1}{2}$ and $L$ in (v) is given by (7.17), if

$$
\begin{gathered}
1 \gtrsim\left(\log \left(s \epsilon^{-1}\right)+1\right) \cdot \frac{N-N_{1}}{m_{2}} \cdot\left(\kappa_{\mathbf{N}, \mathbf{M}}(2,1)+\kappa_{\mathbf{N}, \mathbf{M}}(2,2)\right) \cdot \log (K M \sqrt{s}), \\
m_{2} \gtrsim\left(\log \left(s \epsilon^{-1}\right)+1\right) \cdot \hat{m}_{2} \cdot \log (K M \sqrt{s}),
\end{gathered}
$$

where $\hat{m}_{2}$ satisfies $1 \gtrsim\left(\left(N_{2}-N_{1}\right) / \hat{m}_{2}-1\right) \cdot \mu_{N_{1}} \cdot \tilde{s}_{2}$, and $\tilde{s}_{2} \leqslant S_{2}$ (recall $S_{2}$ from Definition 4.4). Recall from (7.20) that

$$
\kappa_{\mathbf{N}, \mathbf{M}}(2,1) \leqslant \sqrt{s_{1} \cdot \mu_{N_{1}}} \cdot\left\|P_{N_{1}}^{\perp} U P_{M_{1}}\right\|, \quad \kappa_{\mathbf{N}, \mathbf{M}}(2,2) \leqslant s_{2} \cdot \mu_{N_{1}} .
$$


Also, it follows directly from Definition 4.4 that

$$
S_{2} \leqslant\left(\left\|P_{N_{1}}^{\perp} U P_{M_{1}}\right\| \cdot \sqrt{M_{1}}+\sqrt{s_{2}}\right)^{2} .
$$

Thus, provided that $\left\|P_{N_{1}}^{\perp} U P_{M_{1}}\right\| \leqslant \gamma / \sqrt{M_{1}}$ where $\gamma$ is as in (i) of Theorem 5.2, we observe that (iii) of Theorem 5.2 implies (7.22) and (7.23). Thus, the theorem now follows from Proposition 7.1.

Proof of Theorems 4.5 and 5.3. It is straightforward that Theorem 4.5 follows from Theorem 5.3. Now, recall from Lemma 7.20 that

$\kappa_{\mathbf{N}, \mathbf{M}}(k, l) \leqslant s_{l} \cdot \mu_{\mathbf{N}, \mathbf{M}}(k, l), \quad \kappa_{\mathbf{N}, \mathbf{M}}(k, \infty) \leqslant s_{r} \cdot \mu_{\mathbf{N}, \mathbf{M}}(k, \infty), \quad k, l=1, \ldots, r$.

Thus, a direct application of Propositions 7.4 and 7.1 completes the proof.

It remains now to prove Propositions 7.3 and 7.4. This is the content of the next sections.

7.2. Preliminaries. Before we commence on the rather length proof of these propositions, let us recall one of the monumental results in probability theory that will be of greater use later on.

THEOREM 7.6 (Talagrand [59, 79]). There exists a number $K$ with the following property. Consider $n$ independent random variables $X_{i}$ valued in a measurable space $\Omega$ and let $\mathcal{F}$ be a (countable) class of measurable functions on $\Omega$. Let $Z$ be the random variable $Z=\sup _{f \in \mathcal{F}} \sum_{i \leqslant n} f\left(X_{i}\right)$ and define

$$
S=\sup _{f \in \mathcal{F}}\|f\|_{\infty}, \quad V=\sup _{f \in \mathcal{F}} \mathbb{E}\left(\sum_{i \leqslant n} f\left(X_{i}\right)^{2}\right) .
$$

If $\mathbb{E}\left(f\left(X_{i}\right)\right)=0$ for all $f \in \mathcal{F}$ and $i \leqslant n$, then, for each $t>0$, we have

$$
\mathbb{P}(|Z-\mathbb{E}(Z)| \geqslant t) \leqslant 3 \exp \left(-\frac{1}{K} \frac{t}{S} \log \left(1+\frac{t S}{V+S \mathbb{E}(\bar{Z})}\right)\right),
$$

where $\bar{Z}=\sup _{f \in \mathcal{F}}\left|\sum_{i \leqslant n} f\left(X_{i}\right)\right|$.

Note that this version of Talagrand's theorem is found in [59, Corollary 7.8]. We next present a theorem and several technical propositions that will serve as the main tools in our proofs of Propositions 7.3 and 7.4. A crucial tool herein is the Bernoulli sampling model. We use the notation $\{a, \ldots, b\} \supset \Omega \sim \operatorname{Ber}(q)$, where $a<b a, b \in \mathbb{N}$, when $\Omega$ is given by $\Omega=\left\{k: \delta_{k}=1\right\}$ and $\left\{\delta_{k}\right\}_{k=1}^{N}$ is a sequence of Bernoulli variables with $\mathbb{P}\left(\delta_{k}=1\right)=q$. 
Definition 7.7. Let $r \in \mathbb{N}, \mathbf{N}=\left(N_{1}, \ldots, N_{r}\right) \in \mathbb{N}^{r}$ with $1 \leqslant N_{1}<\cdots<N_{r}$, $\mathbf{m}=\left(m_{1}, \ldots, m_{r}\right) \in \mathbb{N}^{r}$, with $m_{k} \leqslant N_{k}-N_{k-1}, k=1, \ldots, r$, and suppose that

$$
\Omega_{k} \subseteq\left\{N_{k-1}+1, \ldots, N_{k}\right\}, \quad \Omega_{k} \sim \operatorname{Ber}\left(\frac{m_{k}}{N_{k}-N_{k-1}}\right), \quad k=1, \ldots, r,
$$

where $N_{0}=0$. We refer to the set $\Omega=\Omega_{\mathbf{N}, \mathbf{m}}:=\Omega_{1} \cup \cdots \cup \Omega_{r}$. as an (N, m)multilevel Bernoulli sampling scheme.

THEOREM 7.8. Let $U \in \mathcal{B}\left(l^{2}(\mathbb{N})\right)$ be an isometry. Suppose that $\Omega=\Omega_{\mathbf{N}, \mathbf{m}}$ is a multilevel Bernoulli sampling scheme, where $\mathbf{N}=\left(N_{1}, \ldots, N_{r}\right) \in \mathbb{N}^{r}$ and $\mathbf{m}=\left(m_{1}, \ldots, m_{r}\right) \in \mathbb{N}^{r}$. Consider $(\mathbf{s}, \mathbf{M})$, where $\mathbf{M}=\left(M_{1}, \ldots, M_{r}\right) \in \mathbb{N}^{r}$, $M_{1}<\cdots<M_{r}$, and $\mathbf{s}=\left(s_{1}, \ldots, s_{r}\right) \in \mathbb{N}^{r}$, and let

$$
\Delta=\Delta_{1} \cup \cdots \cup \Delta_{r}, \quad \Delta_{k} \subset\left\{M_{k-1}+1, \ldots, M_{k}\right\}, \quad\left|\Delta_{k}\right|=s_{k}
$$

where $M_{0}=0$. If $\left\|P_{M_{r}} U^{*} P_{N_{r}} U P_{M_{r}}-P_{M_{r}}\right\| \leqslant 1 / 8$ then, for $\gamma \in(0,1)$,

$$
\mathbb{P}\left(\left\|P_{\Delta} U^{*}\left(q_{1}^{-1} P_{\Omega_{1}} \oplus \cdots \oplus q_{r}^{-1} P_{\Omega_{r}}\right) U P_{\Delta}-P_{\Delta}\right\| \geqslant 1 / 4\right) \leqslant \gamma,
$$

where $q_{k}=m_{k} /\left(N_{k}-N_{k-1}\right)$, provided that

$$
1 \gtrsim \frac{N_{k}-N_{k-1}}{m_{k}} \cdot\left(\sum_{l=1}^{r} \kappa_{\mathbf{N}, \mathbf{M}}(k, l)\right) \cdot\left(\log \left(\gamma^{-1} s\right)+1\right) .
$$

In addition, if $q=\min \left\{q_{k}\right\}_{k=1}^{r}=1$ then

$$
\mathbb{P}\left(\left\|P_{\Delta} U^{*}\left(q_{1}^{-1} P_{\Omega_{1}} \oplus \cdots \oplus q_{r}^{-1} P_{\Omega_{r}}\right) U P_{\Delta}-P_{\Delta}\right\| \geqslant 1 / 4\right)=0 .
$$

In proving this theorem we deliberately avoid the use of the Matrix Bernstein inequality [43], as Talagrand's theorem is more convenient for our infinitedimensional setting. Before we can prove this theorem, we need the following technical lemma.

LEMMA 7.9. Let $U \in \mathcal{B}\left(l^{2}(\mathbb{N})\right)$ with $\|U\| \leqslant 1$, and consider the setup in Theorem 7.8. Let $N=N_{r}$ and let $\left\{\delta_{j}\right\}_{j=1}^{N}$ be independent random Bernoulli variables with $\mathbb{P}\left(\delta_{j}=1\right)=\tilde{q}_{j}, \tilde{q}_{j}=m_{k} /\left(N_{k}-N_{k-1}\right)$ and $j \in\left\{N_{k-1}+1, \ldots, N_{k}\right\}$, and define $Z=\sum_{j=1}^{N} Z_{j}, Z_{j}=\left(\tilde{q}_{j}^{-1} \delta_{j}-1\right) \eta_{j} \otimes \bar{\eta}_{j}$ and $\eta_{j}=P_{\Delta} U^{*} e_{j}$. Then

$$
\mathbb{E}(\|Z\|)^{2} \leqslant 48 \max \{\log (|\Delta|), 1\} \max _{1 \leqslant j \leqslant N}\left\{\tilde{q}_{j}^{-1}\left\|\eta_{j}\right\|^{2}\right\},
$$

when $(\max \{\log (|\Delta|), 1\})^{-1} \geqslant 18 \max _{1 \leqslant j \leqslant N}\left\{\tilde{q}_{j}^{-1}\left\|\eta_{j}\right\|^{2}\right\}$. 
The proof of this lemma involves essentially reworking an argument due to Rudelson [75], and is similar to arguments given previously in [3] (see also [17]). We include it here for completeness as the setup deviates slightly. We shall also require the following result:

LEMMA 7.10 (Rudelson). Let $\eta_{1}, \ldots, \eta_{M} \in \mathbb{C}^{n}$ and let $\varepsilon_{1}, \ldots, \varepsilon_{M}$ be independent Bernoulli variables taking values $1,-1$ with probability $1 / 2$. Then

$$
\mathbb{E}\left(\left\|\sum_{i=1}^{M} \varepsilon_{i} \bar{\eta}_{i} \otimes \eta_{i}\right\|\right) \leqslant \frac{3}{2} \sqrt{p} \max _{i \leqslant M}\left\|\eta_{i}\right\| \sqrt{\left\|\sum_{i=1}^{M} \bar{\eta}_{i} \otimes \eta_{i}\right\|},
$$

where $p=\max \{2,2 \log (n)\}$.

Lemma 7.10 is often referred to as Rudelson's Lemma [75]. However, we use the above complex version that was proven by Tropp [81, Lemma 22].

Proof of Lemma 7.9. We commence by letting $\tilde{\delta}=\left\{\tilde{\delta}_{j}\right\}_{j=1}^{N}$ be independent copies of $\delta=\left\{\delta_{j}\right\}_{j=1}^{N}$. Then, since $\mathbb{E}(Z)=0$,

$$
\begin{aligned}
\mathbb{E}_{\delta}(\|Z\|) & =\mathbb{E}_{\delta}\left(\left\|Z-\mathbb{E}_{\tilde{\delta}}\left(\sum_{j=1}^{N}\left(\tilde{q}_{j}^{-1} \tilde{\delta}_{j}-1\right) \eta_{j} \otimes \bar{\eta}_{j}\right)\right\|\right) \\
& \leqslant \mathbb{E}_{\delta}\left(\mathbb{E}_{\tilde{\delta}}\left(\left\|Z-\sum_{j=1}^{N}\left(\tilde{q}_{j}^{-1} \tilde{\delta}_{j}-1\right) \eta_{j} \otimes \bar{\eta}_{j}\right\|\right)\right),
\end{aligned}
$$

by Jensen's inequality. Let $\varepsilon=\left\{\varepsilon_{j}\right\}_{j=1}^{N}$ be a sequence of Bernoulli variables taking values \pm 1 with probability $1 / 2$. Then, by (7.26), symmetry, Fubini's theorem and the triangle inequality, it follows that

$$
\begin{aligned}
\mathbb{E}_{\delta}(\|Z\|) & \leqslant \mathbb{E}_{\varepsilon}\left(\mathbb{E}_{\delta}\left(\mathbb{E}_{\tilde{\delta}}\left(\left\|\sum_{j=1}^{N} \varepsilon_{j}\left(\tilde{q}_{j}^{-1} \delta_{j}-\tilde{q}_{j}^{-1} \tilde{\delta}_{j}\right) \eta_{j} \otimes \bar{\eta}_{j}\right\|\right)\right)\right) \\
& \leqslant 2 \mathbb{E}_{\delta}\left(\mathbb{E}_{\varepsilon}\left(\left\|\sum_{j=1}^{N} \varepsilon_{j} \tilde{q}_{j}^{-1} \delta_{j} \eta_{j} \otimes \bar{\eta}_{j}\right\|\right)\right) .
\end{aligned}
$$

We are now able to apply Rudelson's lemma (Lemma 7.10). However, as specified before, it is the complex version that is crucial here. By Lemma 7.10 we get that 


$$
\begin{aligned}
\mathbb{E}_{\varepsilon}\left(\left\|\sum_{j=1}^{N} \varepsilon_{j} \tilde{q}_{j}^{-1} \delta_{j} \eta_{j} \otimes \bar{\eta}_{j}\right\|\right) \leqslant & \frac{3}{2} \sqrt{\max \{2 \log (s), 2\}} \\
& \times \max _{1 \leqslant j \leqslant N} \tilde{q}_{j}^{-1 / 2}\left\|\eta_{j}\right\| \sqrt{\left\|\sum_{j=1}^{N} q_{j}^{-1} \tilde{q}_{j}^{-1} \delta_{j} \eta_{j} \otimes \bar{\eta}_{j}\right\|},
\end{aligned}
$$

where $s=|\Delta|$. And hence, by using (7.27) and (7.28), it follows that

$$
\mathbb{E}_{\delta}(\|Z\|) \leqslant 3 \sqrt{\max \{2 \log (s), 2\}} \max _{1 \leqslant j \leqslant N} \tilde{q}_{j}^{-1 / 2}\left\|\eta_{j}\right\| \sqrt{\mathbb{E}_{\delta}\left(\left\|Z+\sum_{j=1}^{N} \eta_{j} \otimes \bar{\eta}_{j}\right\|\right)} .
$$

Note that $\left\|\sum_{j=1}^{N} \eta_{j} \otimes \bar{\eta}_{j}\right\| \leqslant 1$, since $U$ is an isometry. The result now follows from the straightforward calculus fact that if $r>0, c \leqslant 1$ and $r \leqslant c \sqrt{r+1}$ then we have that $r \leqslant c(1+\sqrt{5}) / 2$.

Proof of Theorem 7.8. Let $N=N_{r}$ just to be clear here. Let $\left\{\delta_{j}\right\}_{j=1}^{N}$ be random Bernoulli variables as defined in Lemma 7.9 and define $Z=\sum_{j=1}^{N} Z_{j}$, $Z_{j}=\left(\tilde{q}_{j}^{-1} \delta_{j}-1\right) \eta_{j} \otimes \bar{\eta}_{j}$ with $\eta_{j}=P_{\Delta} U^{*} e_{j}$. Now observe that

$$
\begin{gathered}
P_{\Delta} U^{*}\left(q_{1}^{-1} P_{\Omega_{1}} \oplus \cdots \oplus q_{r}^{-1} P_{\Omega_{r}}\right) U P_{\Delta}=\sum_{j=1}^{N} \tilde{q}_{j}^{-1} \delta_{j} \eta_{j} \otimes \bar{\eta}_{j} \\
P_{\Delta} U^{*} P_{N} U P_{\Delta}=\sum_{j=1}^{N} \eta_{j} \otimes \bar{\eta}_{j}
\end{gathered}
$$

Thus, it follows that

$$
\begin{aligned}
& \left\|P_{\Delta} U^{*}\left(q_{1}^{-1} P_{\Omega_{1}} \oplus \cdots \oplus q_{r}^{-1} P_{\Omega_{r}}\right) U P_{\Delta}-P_{\Delta}\right\| \\
& \quad \leqslant\|Z\|+\left\|\left(P_{\Delta} U^{*} P_{N} U P_{\Delta}-P_{\Delta}\right)\right\| \leqslant\|Z\|+\frac{1}{8},
\end{aligned}
$$

by the assumption that $\left\|P_{M_{r}} U^{*} P_{N_{r}} U P_{M_{r}}-P_{M_{r}}\right\| \leqslant 1 / 8$. Thus, to prove the assertion we need to estimate $\|Z\|$, and Talagrand's theorem (Theorem 7.6) will be our main tool. Note that clearly, since $Z$ is self-adjoint, we have that $\|Z\|=\sup _{\zeta \in \mathcal{G}}|\langle Z \zeta, \zeta\rangle|$, where $\mathcal{G}$ is a countable set of vectors in the unit ball of $P_{\Delta}(\mathcal{H})$. For $\zeta \in \mathcal{G}$ define the mappings

$$
\hat{\zeta}_{1}(T)=\langle T \zeta, \zeta\rangle, \quad \hat{\zeta}_{2}(T)=-\langle T \zeta, \zeta\rangle, \quad T \in \mathcal{B}(\mathcal{H})
$$


In order to use Talagrand's Theorem 7.6 we restrict the domain $\mathcal{D}$ of the mappings $\zeta_{i}$ to

$$
\mathcal{D}=\left\{T \in \mathcal{B}(\mathcal{H}):\|T\| \leqslant \max _{1 \leqslant j \leqslant N}\left\{\tilde{q}_{j}^{-1}\left\|\eta_{j}\right\|^{2}\right\}\right\} .
$$

Let $\mathcal{F}$ denote the family of mappings $\hat{\zeta}_{1}, \hat{\zeta}_{2}$ for $\zeta \in \mathcal{G}$. Then $\|Z\|=\sup _{\hat{\zeta} \in \mathcal{F}} \hat{\zeta}(Z)$, and for $i=1,2$ we have

$$
\left|\hat{\zeta}_{i}\left(Z_{j}\right)\right|=\left|\left(\tilde{q}_{j}^{-1} \delta_{j}-1\right)\right|\left|\left\langle\left(\eta_{j} \otimes \bar{\eta}_{j}\right) \zeta, \zeta\right\rangle\right| \leqslant \max _{1 \leqslant j \leqslant N}\left\{\tilde{q}_{j}^{-1}\left\|\eta_{j}\right\|^{2}\right\} .
$$

Thus, $Z_{j} \in \mathcal{D}$ for $1 \leqslant j \leqslant N$ and $S:=\sup _{\zeta \in \mathcal{F}}\|\hat{\zeta}\|_{\infty}=\max _{1 \leqslant j \leqslant N}\left\{\tilde{q}_{j}^{-1}\left\|\eta_{j}\right\|^{2}\right\}$. Note that

$$
\left\|\eta_{j}\right\|^{2}=\left\langle P_{\Delta} U^{*} e_{j}, P_{\Delta} U^{*} e_{j}\right\rangle=\sum_{k=1}^{r}\left\langle P_{\Delta_{k}} U^{*} e_{j}, P_{\Delta_{k}} U^{*} e_{j}\right\rangle .
$$

Also, note that an easy application of Holder's inequality gives the following (note that the $l^{1}$ and $l^{\infty}$ bounds are finite because all the projections have finite rank),

$$
\begin{aligned}
\left|\left\langle P_{\Delta_{k}} U^{*} e_{j}, P_{\Delta_{k}} U^{*} e_{j}\right\rangle\right| & \leqslant\left\|P_{\Delta_{k}} U^{*} e_{j}\right\|_{l^{1}}\left\|P_{\Delta_{k}} U^{*} e_{j}\right\|_{l^{\infty}} \\
& \leqslant\left\|P_{\Delta_{k}} U^{*} P_{N_{l}}^{N_{l-1}}\right\|_{l^{1} \rightarrow l^{1}}\left\|P_{\Delta_{k}} U^{*} e_{j}\right\|_{l^{\infty}} \\
& \leqslant\left\|P_{N_{l}}^{N_{l-1}} U P_{\Delta_{k}}\right\|_{l^{\infty} \rightarrow l^{\infty}} \cdot \sqrt{\mu\left(P_{N_{l}}^{N_{l-1}} U\right)} \leqslant \kappa_{\mathbf{N}, \mathbf{M}}(l, k),
\end{aligned}
$$

for $j \in\left\{N_{l-1}+1, \ldots, N_{l}\right\}$ and $l \in\{1, \ldots, r\}$. Hence, it follows that

$$
\left\|\eta_{j}\right\|^{2} \leqslant \max _{1 \leqslant k \leqslant r}\left(\kappa_{\mathbf{N}, \mathbf{M}}(k, 1)+\cdots+\kappa_{\mathbf{N}, \mathbf{M}}(k, r)\right),
$$

and therefore $S \leqslant \max _{1 \leqslant k \leqslant r}\left(q_{k}^{-1} \sum_{j=1}^{r} \kappa_{\mathbf{N}, \mathbf{M}}(k, j)\right)$. Finally, note that by (7.31) and the reasoning above, it follows that

$$
\begin{aligned}
V & :=\sup _{\hat{\zeta}_{i} \in \mathcal{F}} \mathbb{E}\left(\sum_{j=1}^{N} \hat{\zeta}_{i}\left(Z_{j}\right)^{2}\right)=\sup _{\zeta \in \mathcal{G}} \mathbb{E}\left(\sum_{j=1}^{N}\left(\tilde{q}_{j}^{-1} \delta_{j}-1\right)^{2}\left|\left\langle P_{\Delta} U^{*} e_{j}, \zeta\right\rangle\right|^{4}\right) \\
& \leqslant \max _{1 \leqslant k \leqslant r}\left\|\eta_{k}\right\|^{2}\left(\frac{N_{k}-N_{k-1}}{m_{k}}-1\right) \sup _{\zeta \in \mathcal{G}} \sum_{j=1}^{N}\left|\left\langle e_{j}, U P_{\Delta} \zeta\right\rangle\right|^{2}, \\
& \leqslant \max _{1 \leqslant k \leqslant r} \frac{N_{k}-N_{k-1}}{m_{k}}\left(\sum_{l=1}^{r} \kappa_{\mathbf{N}, \mathbf{M}}(k, l)\right) \sup _{\zeta \in \mathcal{G}}\|U \zeta\|^{2} \\
& =\max _{1 \leqslant k \leqslant r} \frac{N_{k}-N_{k-1}}{m_{k}}\left(\sum_{l=1}^{r} \kappa_{\mathbf{N}, \mathbf{M}}(k, l)\right),
\end{aligned}
$$


where we used the fact that $U$ is an isometry to deduce that $\|U\|=1$. Also, by Lemma 7.9 and (7.31), it follows that

$$
\mathbb{E}(\|Z\|)^{2} \leqslant 48 \max _{1 \leqslant k \leqslant r} \frac{N_{k}-N_{k-1}}{m_{k}}\left(\sum_{l=1}^{r} \kappa_{\mathbf{N}, \mathbf{M}}(k, l)\right) \cdot \log (s)
$$

when

$$
1 \geqslant 18 \max _{1 \leqslant k \leqslant r} \frac{N_{k}-N_{k-1}}{m_{k}}\left(\sum_{l=1}^{r} \kappa_{\mathbf{N}, \mathbf{M}}(k, l)\right) \cdot \log (s),
$$

(recall that we have assumed $s \geqslant 3$ ). Thus, by (7.30) and Talagrand's Theorem 7.6, it follows that

$$
\begin{aligned}
& \mathbb{P}\left(\left\|P_{\Delta} U^{*}\left(q_{1}^{-1} P_{\Omega_{1}} \oplus \cdots \oplus q_{r}^{-1} P_{\Omega_{r}}\right) U P_{\Delta}-P_{\Delta}\right\| \geqslant 1 / 4\right) \\
& \quad \leqslant \mathbb{P}\left(\|Z\| \geqslant \frac{1}{16}+\sqrt{\left.24 \max _{1 \leqslant k \leqslant r} \frac{N_{k}-N_{k-1}}{m_{k}}\left(\sum_{l=1}^{r} \kappa_{\mathbf{N}, \mathbf{M}}(k, l)\right) \cdot \log (s)\right)}\right. \\
& \quad \leqslant 3 \exp \left(-\frac{1}{16 K}\left(\max _{1 \leqslant k \leqslant r} \frac{N_{k}-N_{k-1}}{m_{k}}\left(\sum_{l=1}^{r} \kappa_{\mathbf{N}, \mathbf{M}}(k, l)\right)\right)^{-1} \log (1+1 / 32)\right),
\end{aligned}
$$

when $m_{k}$ 's are chosen such that the right hand side of (7.33) is less than or equal to 1 . Thus, by (7.30) and Talagrand's Theorem 7.6, it follows that

$$
\begin{aligned}
& \mathbb{P}\left(\left\|P_{\Delta} U^{*}\left(q_{1}^{-1} P_{\Omega_{1}} \oplus \cdots \oplus q_{r}^{-1} P_{\Omega_{r}}\right) U P_{\Delta}-P_{\Delta}\right\| \geqslant 1 / 4\right) \\
& \quad \leqslant \mathbb{P}(\|Z\| \geqslant 1 / 8) \leqslant \mathbb{P}\left(\|Z\| \geqslant \frac{1}{16}+\mathbb{E}\|Z\|\right) \leqslant \mathbb{P}\left(|\|Z\|-\mathbb{E}\|Z\|| \geqslant \frac{1}{16}\right) \\
& \quad \leqslant 3 \exp \left(-\frac{1}{16 K}\left(\max _{1 \leqslant k \leqslant r} \frac{N_{k}-N_{k-1}}{m_{k}}\left(\sum_{l=1}^{r} \kappa_{\mathbf{N}, \mathbf{M}}(k, l)\right)\right)^{-1} \log (1+1 / 32)\right),
\end{aligned}
$$

when $m_{k}$ 's are chosen such that the right hand side of (7.33) is less than or equal to $1 / 16^{2}$. Note that this condition is implied by the assumptions of the theorem as is (7.34). This yields the first part of the theorem. The second claim of this theorem follows from the assumption that $\left\|P_{M_{r}} U^{*} P_{N_{r}} U P_{M_{r}}-P_{M_{r}}\right\| \leqslant 1 / 8$.

Proposition 7.11. Let $U \in \mathcal{B}\left(l^{2}(\mathbb{N})\right)$ be an isometry. Suppose that $\Omega=\Omega_{\mathbf{N}, \mathbf{m}}$ is a multilevel Bernoulli sampling scheme, where $\mathbf{N}=\left(N_{1}, \ldots, N_{r}\right) \in \mathbb{N}^{r}$ and 
$\mathbf{m}=\left(m_{1}, \ldots, m_{r}\right) \in \mathbb{N}^{r}$. Consider $(\mathbf{s}, \mathbf{M})$, where $\mathbf{M}=\left(M_{1}, \ldots, M_{r}\right) \in \mathbb{N}^{r}$, $M_{1}<\cdots<M_{r}$, and $\mathbf{s}=\left(s_{1}, \ldots, s_{r}\right) \in \mathbb{N}^{r}$, and let $\Delta=\Delta_{1} \cup \cdots \cup \Delta_{r}$, $\Delta_{k} \subset\left\{M_{k-1}, \ldots, M_{k}\right\},\left|\Delta_{k}\right|=s_{k}$, where $M_{0}=0$. Let $\beta \geqslant 1 / 4$.

(i) If

$$
N:=N_{r}, \quad K:=\max _{k=1, \ldots, r}\left\{\frac{N_{k}-N_{k-1}}{m_{k}}\right\},
$$

satisfy the weak balancing property with respect to $U, M:=M_{r}$ and $s:=s_{1}+\cdots+s_{r}$, then, for $\xi \in \mathcal{H}$ and $\beta, \gamma>0$, we have that

$$
\mathbb{P}\left(\left\|P_{M} P_{\Delta}^{\perp} U^{*}\left(q_{1}^{-1} P_{\Omega_{1}} \oplus \cdots \oplus q_{r}^{-1} P_{\Omega_{r}}\right) U P_{\Delta} \xi\right\|_{l^{\infty}}>\beta\|\xi\|_{l^{\infty}}\right) \leqslant \gamma
$$

provided that

$$
\frac{\beta}{\log \left(\frac{4}{\gamma}(M-s)\right)} \geqslant C \Lambda, \quad \frac{\beta^{2}}{\log \left(\frac{4}{\gamma}(M-s)\right)} \geqslant C \Upsilon,
$$

for some constant $C>0$, where $q_{k}=m_{k} /\left(N_{k}-N_{k-1}\right)$ for $k=1, \ldots, r$,

$$
\begin{gathered}
\Lambda=\max _{1 \leqslant k \leqslant r}\left\{\frac{N_{k}-N_{k-1}}{m_{k}} \cdot\left(\sum_{l=1}^{r} \kappa_{\mathbf{N}, \mathbf{M}}(k, l)\right)\right\}, \\
\Upsilon=\max _{1 \leqslant l \leqslant r} \sum_{k=1}^{r}\left(\frac{N_{k}-N_{k-1}}{m_{k}}-1\right) \cdot \mu_{\mathbf{N}, \mathbf{M}}(k, l) \cdot \tilde{s}_{k},
\end{gathered}
$$

for all $\left\{\tilde{s}_{k}\right\}_{k=1}^{r}$ such that $\tilde{s}_{1}+\cdots+\tilde{s}_{r} \leqslant s_{1}+\cdots+s_{r}$ and $\tilde{s}_{k} \leqslant S_{k}\left(s_{1}, \ldots, s_{r}\right)$. Moreover, if $q_{k}=1$ for all $k=1, \ldots, r$, then (7.38) is trivially satisfied for any $\gamma>0$ and the left-hand side of (7.37) is equal to zero.

(ii) If $N$ satisfies the strong Balancing Property with respect to $U, M$ and $s$, then, for $\xi \in \mathcal{H}$ and $\beta, \gamma>0$, we have that

$$
\mathbb{P}\left(\left\|P_{\Delta}^{\perp} U^{*}\left(q_{1}^{-1} P_{\Omega_{1}} \oplus \cdots \oplus q_{r}^{-1} P_{\Omega_{r}}\right) U P_{\Delta} \xi\right\|_{l^{\infty}}>\beta\|\xi\|_{l^{\infty}}\right) \leqslant \gamma,
$$

provided that

$$
\frac{\beta}{\log (4 / \gamma(\tilde{\theta}-s))} \geqslant C \Lambda, \quad \frac{\beta^{2}}{\log (4 / \gamma(\tilde{\theta}-s))} \geqslant C \Upsilon
$$

for some constant $C>0, \tilde{\theta}=\tilde{\theta}\left(\left\{q_{k}\right\}_{k=1}^{r}, 1 / 8,\left\{N_{k}\right\}_{k=1}^{r}, s, M\right)$ and $\Upsilon, \Lambda$ as defined in (i) and 


$$
\begin{aligned}
& \tilde{\theta}\left(\left\{q_{k}\right\}_{k=1}^{r}, t,\left\{N_{k}\right\}_{k=1}^{r}, s, M\right) \\
& =\mid\{i \in \mathbb{N}: \max _{\substack{\Gamma_{1} \subset\{1, \ldots, M\}, \Gamma_{2, j} \subset\left\{N_{j-1}+1, \ldots, N_{j}\right\},}} \underbrace{}_{\substack{\mid=s \\
j=1, \ldots, r}} \\
& \left.\left\|P_{\Gamma_{1}} U^{*}\left(q_{1}^{-1} P_{\Gamma_{2,1}} \oplus \cdots \oplus q_{r}^{-1} P_{\Gamma_{2, r}}\right) U e_{i}\right\|>\frac{t}{\sqrt{s}}\right\} \mid .
\end{aligned}
$$

Moreover, if $q_{k}=1$ for all $k=1, \ldots, r$, then (7.42) is trivially satisfied for any $\gamma>0$ and the left-hand side of (7.41) is equal to zero.

Proof. To prove (i) we note that, without loss of generality, we can assume that $\|\xi\|_{l^{\infty}}=1$. Let $\left\{\delta_{j}\right\}_{j=1}^{N}$ be random Bernoulli variables with $\mathbb{P}\left(\delta_{j}=1\right)=\tilde{q}_{j}=q_{k}$, for $j \in\left\{N_{k-1}+1, \ldots, N_{k}\right\}$ and $1 \leqslant k \leqslant r$. A key observation that will be crucial below is that

$$
\begin{gathered}
P_{\Delta}^{\perp} U^{*}\left(q_{1}^{-1} P_{\Omega_{1}} \oplus \cdots \oplus q_{r}^{-1} P_{\Omega_{r}}\right) U P_{\Delta} \xi=\sum_{j=1}^{N} P_{\Delta}^{\perp} U^{*} \tilde{q}_{j}^{-1} \delta_{j}\left(e_{j} \otimes e_{j}\right) U P_{\Delta} \xi \\
=\sum_{j=1}^{N} P_{\Delta}^{\perp} U^{*}\left(\tilde{q}_{j}^{-1} \delta_{j}-1\right)\left(e_{j} \otimes e_{j}\right) U P_{\Delta} \xi+P_{\Delta}^{\perp} U^{*} P_{N} U P_{\Delta} \xi
\end{gathered}
$$

We use this equation at the end of the argument, but first we estimate the size of the individual components of $\sum_{j=1}^{N} P_{\Delta}^{\perp} U^{*}\left(\tilde{q}_{j}^{-1} \delta_{j}-1\right)\left(e_{j} \otimes e_{j}\right) U P_{\Delta} \xi$. To do that define, for $1 \leqslant j \leqslant N$, the random variables

$$
X_{j}^{i}=\left\langle U^{*}\left(\tilde{q}_{j}^{-1} \delta_{j}-1\right)\left(e_{j} \otimes e_{j}\right) U P_{\Delta} \xi, e_{i}\right\rangle, \quad i \in \Delta^{c} .
$$

We show using Bernstein's inequality that, for each $i \in \Delta^{c}$ and $t>0$,

$$
\mathbb{P}\left(\left|\sum_{j=1}^{N} X_{j}^{i}\right|>t\right) \leqslant 4 \exp \left(-\frac{t^{2} / 4}{\Upsilon+\Lambda t / 3}\right)
$$

To prove the claim, we need to estimate $\mathbb{E}\left(\left|X_{j}^{i}\right|^{2}\right)$ and $\left|X_{j}^{i}\right|$. First note that,

$$
\mathbb{E}\left(\left|X_{j}^{i}\right|^{2}\right)=\left(\tilde{q}_{j}^{-1}-1\right)\left|\left\langle e_{j}, U P_{\Delta} \xi\right\rangle\right|^{2}\left|\left\langle e_{j}, U e_{i}\right\rangle\right|^{2},
$$

and note that $\left|\left\langle e_{j}, U e_{i}\right\rangle\right|^{2} \leqslant \mu_{\mathbf{N}, \mathbf{M}}(k, l)$ for $j \in\left\{N_{k-1}+1, \ldots, N_{k}\right\}$ and $i \in\left\{M_{l-1}+\right.$ $\left.1, \ldots, M_{l}\right\}$. Hence 


$$
\begin{aligned}
\sum_{j=1}^{N} \mathbb{E}\left(\left|X_{j}^{i}\right|^{2}\right) & \leqslant \sum_{k=1}^{r}\left(q_{k}^{-1}-1\right) \mu_{\mathbf{N}, \mathbf{M}}(k, l)\left\|P_{N_{k}}^{N_{k-1}} U P_{\Delta} \xi\right\|^{2} \\
& \leqslant \sup _{\zeta \in \Theta}\left\{\sum_{k=1}^{r}\left(q_{k}^{-1}-1\right) \mu_{\mathbf{N}, \mathbf{M}}(k, l)\left\|P_{N_{k}}^{N_{k-1}} U \zeta\right\|^{2}\right\},
\end{aligned}
$$

where

$$
\Theta=\left\{\eta:\|\eta\|_{l^{\infty}} \leqslant 1,\left|\operatorname{supp}\left(P_{M_{l}}^{M_{l-1}} \eta\right)\right|=s_{l}, l=1, \ldots, r\right\}
$$

The supremum in the above bound is attained for some $\tilde{\zeta} \in \Theta$. If $\tilde{s}_{k}=$ $\left\|P_{N_{k}}^{N_{k-1}} U \tilde{\zeta}\right\|^{2}$, then we have

$$
\sum_{j=1}^{N} \mathbb{E}\left(\left|X_{j}^{i}\right|^{2}\right) \leqslant \sum_{k=1}^{r}\left(q_{k}^{-1}-1\right) \mu_{\mathbf{N}, \mathbf{M}}(k, l) \tilde{s}_{k}
$$

Note that it is clear from the definition that $s_{k} \leqslant S_{k}\left(s_{1}, \ldots, s_{r}\right)$ for $1 \leqslant k \leqslant r$. Also, using the fact that $\|U\| \leqslant 1$ and the definition of $\Theta$, we note that

$$
\tilde{s}_{1}+\cdots+\tilde{s}_{r}=\sum_{k=1}^{r}\left\|P_{N_{k}}^{N_{k-1}} U P_{\Delta} \zeta\right\|^{2} \leqslant\left\|U P_{\Delta} \zeta\right\|^{2}=\|\zeta\|^{2} \leqslant s_{1}+\cdots+s_{r}
$$

To estimate $\left|X_{j}^{i}\right|$ we start by observing that, by the triangle inequality, the fact that $\|\xi\|_{l^{\infty}}=1$ and Holder's inequality, it follows that $\left|\left\langle\xi, P_{\Delta} U^{*} e_{j}\right\rangle\right| \leqslant$ $\sum_{k=1}^{r}\left|\left\langle P_{M_{k}}^{M_{k-1}} \xi, P_{\Delta} U^{*} e_{j}\right\rangle\right|$, and

$$
\begin{gathered}
\left|\left\langle P_{M_{k}}^{M_{k-1}} \xi, P_{\Delta} U^{*} e_{j}\right\rangle\right| \leqslant\left\|P_{N_{l}}^{N_{l-1}} U P_{\Delta_{k}}\right\|_{l^{\infty} \rightarrow l^{\infty}}, \\
j \in\left\{N_{l-1}+1, \ldots, N_{l}\right\}, l \in\{1, \ldots, r\} .
\end{gathered}
$$

Hence, it follows that for $1 \leqslant j \leqslant N$ and $i \in \Delta^{c}$,

$$
\begin{aligned}
\left|X_{j}^{i}\right| & =\tilde{q}_{j}^{-1}\left|\left(\delta_{j}-\tilde{q}_{j}\right)\right|\left|\left\langle\xi, P_{\Delta} U^{*} e_{j}\right\rangle\right|\left|\left\langle e_{j}, U e_{i}\right\rangle\right|, \\
& \leqslant \max _{1 \leqslant k \leqslant r}\left\{\frac{N_{k}-N_{k-1}}{m_{k}} \cdot\left(\kappa_{\mathbf{N}, \mathbf{M}}(k, 1)+\cdots+\kappa_{\mathbf{N}, \mathbf{M}}(k, r)\right)\right\} .
\end{aligned}
$$

Now, clearly $\mathbb{E}\left(X_{j}^{i}\right)=0$ for $1 \leqslant j \leqslant N$ and $i \in \Delta^{c}$. Thus, by applying Bernstein's inequality to $\operatorname{Re}\left(X_{j}^{i}\right)$ and $\operatorname{Im}\left(X_{j}^{i}\right)$ for $j=1, \ldots, N$, via (7.45) and (7.46), the claim (7.44) follows. 
Now, by (7.44), (7.43) and the assumed weak Balancing property (wBP), it follows that

$$
\begin{aligned}
& \mathbb{P}\left(\left\|P_{M} P_{\Delta}^{\perp} U^{*}\left(q_{1}^{-1} P_{\Omega_{1}} \oplus \cdots \oplus q_{r}^{-1} P_{\Omega_{r}}\right) U P_{\Delta} \xi\right\|_{l^{\infty}}>\beta\right) \\
& \leqslant \sum_{i \in \Delta^{c} \cap\{1, \ldots, M\}} \mathbb{P}\left(\left|\sum_{j=1}^{N} X_{j}^{i}+\left\langle P_{M} P_{\Delta}^{\perp} U^{*} P_{N}^{\perp} U P_{\Delta} \xi, e_{i}\right\rangle\right|>\beta\right) \\
& \leqslant \sum_{i \in \Delta^{c} \cap\{1, \ldots, M\}} \mathbb{P}\left(\left|\sum_{j=1}^{N} X_{j}^{i}\right|>\beta-\left\|P_{M} P_{\Delta}^{\perp} U^{*} P_{N} U P_{\Delta}\right\|_{l^{\infty}}\right) \\
& \quad \leqslant 4(M-s) \exp \left(-\frac{t^{2} / 4}{\Upsilon+\Lambda t / 3}\right), \quad t=\frac{1}{2} \beta, \quad \text { by (7.44), (wBP). }
\end{aligned}
$$

Also,

$$
4(M-s) \exp \left(-\frac{t^{2} / 4}{\Upsilon+\Lambda t / 3}\right) \leqslant \gamma
$$

when

$$
\log \left(\frac{4}{\gamma}(M-s)\right)^{-1} \geqslant\left(\frac{4 \Upsilon}{t^{2}}+\frac{4 \Lambda}{3 t}\right)
$$

And this concludes the proof of (i). To prove (ii), for $t>0$, suppose that there is a set $\Lambda_{t} \subset \mathbb{N}$ such that

$$
\mathbb{P}\left(\sup _{i \in \Lambda_{t}}\left|\left\langle P_{\Delta}^{\perp} U^{*}\left(q_{1}^{-1} P_{\Omega_{1}} \oplus \cdots \oplus q_{r}^{-1} P_{\Omega_{r}}\right) U P_{\Delta} \eta, e_{i}\right\rangle\right|>t\right)=0, \quad\left|\Lambda_{t}^{c}\right|<\infty .
$$

Then, as before, by (7.44), (7.43) and the assumed strong Balancing property (sBP), it follows that

$$
\begin{aligned}
& \mathbb{P}\left(\left\|P_{\Delta}^{\perp} U^{*}\left(q_{1}^{-1} P_{\Omega_{1}} \oplus \cdots \oplus q_{r}^{-1} P_{\Omega_{r}}\right) U P_{\Delta} \xi\right\|_{l^{\infty}}>\beta\right) \\
& \quad \leqslant \sum_{i \in \Delta^{c} \cap \Lambda_{t}^{c}} \mathbb{P}\left(\left|\sum_{j=1}^{N} X_{j}^{i}+\left\langle P_{\Delta}^{\perp} U^{*} P_{N}^{\perp} U P_{\Delta} \xi, e_{i}\right\rangle\right|>\beta\right),
\end{aligned}
$$

yielding

$$
\begin{aligned}
& \mathbb{P}\left(\left\|P_{\Delta}^{\perp} U^{*}\left(q_{1}^{-1} P_{\Omega_{1}} \oplus \cdots \oplus q_{r}^{-1} P_{\Omega_{r}}\right) U P_{\Delta} \xi\right\|_{l^{\infty}}>\beta\right) \\
& \quad \leqslant \sum_{i \in \Delta^{c} \cap \Lambda_{t}^{c}} \mathbb{P}\left(\left|\sum_{j=1}^{N} X_{j}^{i}\right|>\beta-\left\|P_{\Delta}^{\perp} U^{*} P_{N} U P_{\Delta}\right\|_{l^{\infty}}\right) \\
& \quad \leqslant 4\left(\left|\Lambda_{t}^{c}\right|-s\right) \exp \left(-\frac{t^{2} / 4}{\Upsilon+\Lambda t / 3}\right)<\gamma, \quad t=\frac{1}{2} \beta, \quad \text { by (7.44), (sBP) }
\end{aligned}
$$


whenever

$$
\log \left(\frac{4}{\gamma}\left(\left|\Lambda_{t}^{c}\right|-s\right)\right)^{-1} \geqslant\left(\frac{4 \Upsilon}{t^{2}}+\frac{4 \Lambda}{3 t}\right) .
$$

Hence, it remains to obtain a bound on $\left|\Lambda_{t}^{c}\right|$. Let

$$
\begin{aligned}
& \theta\left(q_{1}, \ldots, q_{r}, t, s\right)
\end{aligned}
$$

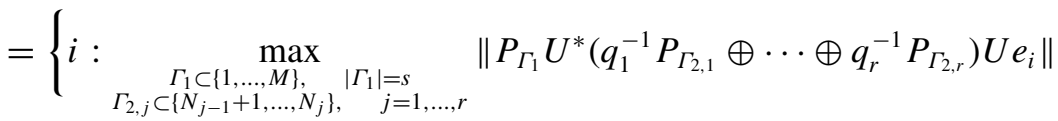

$$
\begin{aligned}
& \left.>\frac{t}{\sqrt{s}}\right\} \text {. }
\end{aligned}
$$

Clearly, $\Delta_{t}^{c} \subset \theta\left(q_{1}, \ldots, q_{r}, t, s\right)$ and

$$
\left\|P_{\Gamma_{1}} U^{*}\left(q_{1}^{-1} P_{\Gamma_{2,1}} \oplus \cdots \oplus q_{r}^{-1} P_{\Gamma_{2, r}}\right) U e_{i}\right\| \leqslant \max _{1 \leqslant j \leqslant r} q_{j}^{-1}\left\|P_{N} U P_{i-1}^{\perp}\right\| \rightarrow 0
$$

as $i \rightarrow \infty$. So, $\left|\theta\left(q_{1}, \ldots, q_{r}, t, s\right)\right|<\infty$. Also, since $\tilde{\theta}\left(\left\{q_{k}\right\}_{k=1}^{r}, t,\left\{N_{k}\right\}_{k=1}^{r}, s, M\right)$ is a decreasing function in $t$, for all $t \geqslant \frac{1}{8}$,

$$
\left|\theta\left(q_{1}, \ldots, q_{r}, t, s\right)\right|<\tilde{\theta}\left(\left\{q_{k}\right\}_{k=1}^{r}, 1 / 8,\left\{N_{k}\right\}_{k=1}^{r}, s, M\right)
$$

thus, we have proved (ii). The statements at the end of (i) and (ii) are clear from the reasoning above.

Proposition 7.12. Consider the same setup as in Proposition 7.11. If $N$ and $K$ satisfy the weak Balancing Property with respect to $U, M$ and $s$, then, for $\xi \in \mathcal{H}$ and $\gamma>0$, we have

$$
\left.\mathbb{P}\left(\| P_{\Delta} U^{*}\left(q_{1}^{-1} P_{\Omega_{1}} \oplus \cdots \oplus q_{r}^{-1} P_{\Omega_{r}}\right) U P_{\Delta}-P_{\Delta}\right) \xi\left\|_{l^{\infty}}>\tilde{\alpha}\right\| \xi \|_{l^{\infty}}\right) \leqslant \gamma
$$

with $\tilde{\alpha}=\left(2 \log _{2}^{1 / 2}(4 \sqrt{s} K M)\right)^{-1}$, provided that

$$
\begin{aligned}
& 1 \gtrsim \Lambda \cdot\left(\log \left(s \gamma^{-1}\right)+1\right) \cdot \log (\sqrt{s} K M), \\
& 1 \gtrsim \Upsilon \cdot\left(\log \left(s \gamma^{-1}\right)+1\right) \cdot \log (\sqrt{s} K M),
\end{aligned}
$$

where $\Lambda$ and $\Upsilon$ are defined in (7.39) and (7.40). Also,

$$
\left.\mathbb{P}\left(\| P_{\Delta} U^{*}\left(q_{1}^{-1} P_{\Omega_{1}} \oplus \cdots \oplus q_{r}^{-1} P_{\Omega_{r}}\right) U P_{\Delta}-P_{\Delta}\right) \xi\left\|_{l^{\infty}}>\frac{1}{2}\right\| \xi \|_{l^{\infty}}\right) \leqslant \gamma
$$

provided that

$$
1 \gtrsim \Lambda \cdot\left(\log \left(s \gamma^{-1}\right)+1\right), \quad 1 \gtrsim \Upsilon \cdot\left(\log \left(s \gamma^{-1}\right)+1\right)
$$


Moreover, if $q_{k}=1$ for all $k=1, \ldots, r$, then the left-hand sides of (7.47) and (7.48) are equal to zero.

Proof. Without loss of generality we may assume that $\|\xi\|_{l^{\infty}}=1$. Let $\left\{\delta_{j}\right\}_{j=1}^{N}$ be random Bernoulli variables with $\mathbb{P}\left(\delta_{j}=1\right)=\tilde{q}_{j}:=q_{k}$, with $j \in\left\{N_{k-1}+1, \ldots\right.$, $\left.N_{k}\right\}$ and $1 \leqslant k \leqslant r$. Let also, for $j \in \mathbb{N}, \eta_{j}=\left(U P_{\Delta}\right)^{*} e_{j}$. Then, after observing that

$$
\begin{aligned}
P_{\Delta} U^{*}\left(q_{1}^{-1} P_{\Omega_{1}} \oplus \cdots \oplus q_{r}^{-1} P_{\Omega_{r}}\right) U P_{\Delta} & =\sum_{j=1}^{N} q_{j}^{-1} \delta_{j} \eta_{j} \otimes \bar{\eta}_{j}, P_{\Delta} U^{*} P_{N} U P_{\Delta} \\
& =\sum_{j=1}^{N} \eta_{j} \otimes \bar{\eta}_{j},
\end{aligned}
$$

it follows immediately that

$$
\begin{aligned}
& P_{\Delta} U^{*}\left(q_{1}^{-1} P_{\Omega_{1}} \oplus \cdots \oplus q_{r}^{-1} P_{\Omega_{r}}\right) U P_{\Delta}-P_{\Delta} \\
& \quad=\sum_{j=1}^{N}\left(\tilde{q}_{j}^{-1} \delta_{j}-1\right) \eta_{j} \otimes \bar{\eta}_{j}-\left(P_{\Delta} U^{*} P_{N} U P_{\Delta}-P_{\Delta}\right) .
\end{aligned}
$$

As in the proof of Proposition 7.11 our goal is to eventually use Bernstein's inequality and the following is therefore a setup for that. Define, for $1 \leqslant j \leqslant N$, the random variables $Z_{j}^{i}=\left\langle\left(\tilde{q}_{j}^{-1} \delta_{j}-1\right)\left(\eta_{j} \otimes \bar{\eta}_{j}\right) \xi, e_{i}\right\rangle$, for $i \in \Delta$. We claim that, for $t>0$,

$$
\mathbb{P}\left(\left|\sum_{j=1}^{N} Z_{j}^{i}\right|>t\right) \leqslant 4 \exp \left(-\frac{t^{2} / 4}{\Upsilon+\Lambda t / 3}\right), \quad i \in \Delta .
$$

Now, clearly $\mathbb{E}\left(Z_{j}^{i}\right)=0$, so we may use Bernstein's inequality. Thus, we need to estimate $\mathbb{E}\left(\left|Z_{j}^{i}\right|^{2}\right)$ and $\left|Z_{j}^{i}\right|$. We start with $\mathbb{E}\left(\left|Z_{j}^{i}\right|^{2}\right)$. Note that

$$
\mathbb{E}\left(\left|Z_{j}^{i}\right|^{2}\right)=\left(\tilde{q}_{j}^{-1}-1\right)\left|\left\langle e_{j}, U P_{\Delta} \xi\right\rangle\right|^{2}\left|\left\langle e_{j}, U e_{i}\right\rangle\right|^{2} .
$$

Thus, we can argue exactly as in the proof of Proposition 7.11 and deduce that

$$
\sum_{j=1}^{N} \mathbb{E}\left(\left|Z_{j}^{i}\right|^{2}\right) \leqslant \sum_{k=1}^{r}\left(q_{k}^{-1}-1\right) \mu_{N_{k-1}} \tilde{s}_{k}
$$

where $s_{k} \leqslant S_{k}\left(s_{1}, \ldots, s_{r}\right)$ for $1 \leqslant k \leqslant r$ and $\tilde{s}_{1}+\cdots+\tilde{s}_{r} \leqslant s_{1}+\cdots+s_{r}$. To estimate $\left|Z_{j}^{i}\right|$ we argue as in the proof of Proposition 7.11 and obtain

$$
\left|Z_{j}^{i}\right| \leqslant \max _{1 \leqslant k \leqslant r}\left\{\frac{N_{k}-N_{k-1}}{m_{k}} \cdot\left(\kappa_{\mathbf{N}, \mathbf{M}}(k, 1)+\cdots+\kappa_{\mathbf{N}, \mathbf{M}}(k, r)\right)\right\} .
$$


Thus, by applying Bernstein's inequality to $\operatorname{Re}\left(Z_{1}^{i}\right), \ldots, \operatorname{Re}\left(Z_{N}^{i}\right)$ and the imaginary part $\operatorname{Im}\left(Z_{1}^{i}\right), \ldots, \operatorname{Im}\left(Z_{N}^{i}\right)$ we obtain, via (7.52) and (7.53) the estimate (7.50), and we have proved the claim.

Now armed with (7.50) we can deduce that, by (7.43) and the assumed weak Balancing property (wBP), it follows that

$$
\begin{aligned}
& \left.\mathbb{P}\left(\| P_{\Delta} U^{*}\left(q_{1}^{-1} P_{\Omega_{1}} \oplus \cdots \oplus q_{r}^{-1} P_{\Omega_{r}}\right) U P_{\Delta}-P_{\Delta}\right) \xi \|_{l^{\infty}}>\tilde{\alpha}\right) \\
& \quad \leqslant \sum_{i \in \Delta} \mathbb{P}\left(\left|\sum_{j=1}^{N} Z_{j}^{i}+\left\langle\left(P_{\Delta} U^{*} P_{N} U P_{\Delta}-P_{\Delta}\right) \xi, e_{i}\right\rangle\right|>\tilde{\alpha}\right) \\
& \quad \leqslant \sum_{i \in \Delta} \mathbb{P}\left(\left|\sum_{j=1}^{N} Z_{j}^{i}\right|>\tilde{\alpha}-\left\|P_{M} U^{*} P_{N} U P_{M}-P_{M}\right\|_{l^{1}}\right), \\
& \quad \leqslant 4 s \exp \left(-\frac{t^{2} / 4}{\Upsilon+\Lambda t / 3}\right), \quad t=\tilde{\alpha}, \quad \text { by (7.50), (wBP). }
\end{aligned}
$$

Also,

$$
4 s \exp \left(-\frac{t^{2} / 4}{\Upsilon+\Lambda t / 3}\right) \leqslant \gamma
$$

when

$$
1 \geqslant\left(\frac{4 \Upsilon}{t^{2}}+\frac{4}{3 t} \Lambda\right) \cdot \log \left(\frac{4 s}{\gamma}\right)
$$

And this gives the first part of the proposition. Also, the fact that the left-hand side of (7.47) is zero when $q_{k}=1$ for $1 \leqslant k \leqslant r$ is clear from (7.55). Note that (ii) follows by arguing exactly as above and replacing $\tilde{\alpha}$ by $\frac{1}{4}$.

Proposition 7.13. Let $U \in \mathcal{B}\left(l^{2}(\mathbb{N})\right)$ such that $\|U\| \leqslant 1$. Suppose that $\Omega=$ $\Omega_{\mathbf{N}, \mathbf{m}}$ is a multilevel Bernoulli sampling scheme, where $\mathbf{N}=\left(N_{1}, \ldots, N_{r}\right) \in \mathbb{N}^{r}$ and $\mathbf{m}=\left(m_{1}, \ldots, m_{r}\right) \in \mathbb{N}^{r}$. Consider $(\mathbf{s}, \mathbf{M})$, where $\mathbf{M}=\left(M_{1}, \ldots, M_{r}\right) \in \mathbb{N}^{r}$, $M_{1}<\cdots<M_{r}$, and $\mathbf{s}=\left(s_{1}, \ldots, s_{r}\right) \in \mathbb{N}^{r}$, and let $\Delta=\Delta_{1} \cup \cdots \cup \Delta_{r}$, where $\Delta_{k} \subset\left\{M_{k-1}+1, \ldots, M_{k}\right\},\left|\Delta_{k}\right|=s_{k}$, and $M_{0}=0$. Then, for any $t \in(0,1)$ and $\gamma \in(0,1)$,

$$
\mathbb{P}\left(\max _{i \in\{1, \ldots, M\} \cap \Delta^{c}}\left\|P_{\{i\}} U^{*}\left(q_{1}^{-1} P_{\Omega_{1}} \oplus \cdots \oplus q_{r}^{-1} P_{\Omega_{r}}\right) U P_{\{i\}}\right\| \geqslant 1+t\right) \leqslant \gamma
$$

provided that

$$
\frac{t^{2}}{4} \geqslant \log \left(\frac{2 M}{\gamma}\right) \cdot \max _{1 \leqslant k \leqslant r}\left\{\left(\frac{N_{k}-N_{k-1}}{m_{k}}-1\right) \cdot \mu_{\mathbf{N}, \mathbf{M}}(k, l)\right\}
$$


for all $l=1, \ldots, r$ when $M=M_{r}$ and for all $l=1, \ldots, r-1, \infty$ when $M>M_{r}$. In addition, if $m_{k}=N_{k}-N_{k-1}$ for each $k=1, \ldots, r$, then

$$
\mathbb{P}\left(\left\|P_{\{i\}} U^{*}\left(q_{1}^{-1} P_{\Omega_{1}} \oplus \cdots \oplus q_{r}^{-1} P_{\Omega_{r}}\right) U P_{\{i\}}\right\| \geqslant 1+t\right)=0, \quad \forall i \in \mathbb{N} .
$$

Proof. Fix $i \in\{1, \ldots, M\}$. Let $\left\{\delta_{j}\right\}_{j=1}^{N}$ be random independent Bernoulli variables with $\mathbb{P}\left(\delta_{j}=1\right)=\tilde{q}_{j}:=q_{k}$ for $j \in\left\{N_{k-1}+1, \ldots, N_{k}\right\}$. Define $Z=\sum_{j=1}^{N} Z_{j}$ and $Z_{j}=\left(\tilde{q}_{j}^{-1} \delta_{j}-1\right)\left|u_{j i}\right|^{2}$. Now observe that

$$
P_{\{i\}} U^{*}\left(q_{1}^{-1} P_{\Omega_{1}} \oplus \cdots \oplus q_{r}^{-1} P_{\Omega_{r}}\right) U P_{\{i\}}=\sum_{j=1}^{N} \tilde{q}_{j}^{-1} \delta_{j}\left|u_{j i}\right|^{2}=\sum_{j=1}^{N} Z_{j}+\sum_{j=1}^{N}\left|u_{j i}\right|^{2},
$$

where we interpret $U$ as the infinite matrix $U=\left\{u_{i j}\right\}_{i, j \in \mathbb{N}}$. Thus, since $\|U\| \leqslant 1$,

$$
\left\|P_{\{i\}} U^{*}\left(q_{1}^{-1} P_{\Omega_{1}} \oplus \cdots \oplus q_{r}^{-1} P_{\Omega_{r}}\right) U P_{\{i\}}\right\| \leqslant\left|\sum_{j=1}^{N} Z_{j}\right|+1
$$

and it is clear that (7.57) is true. For the case where $q_{k}<1$ for some $k \in\{1, \ldots, r\}$, observe that for $i \in\left\{M_{l-1}+1, \ldots, M_{l}\right\}$ (recall that $Z_{j}$ depend on $i$ ), we have that $\mathbb{E}\left(Z_{j}\right)=0$. Also,

$$
\left|Z_{j}\right| \leqslant\left\{\begin{aligned}
\max _{1 \leqslant k \leqslant r}\left\{\max \left\{q_{k}^{-1}-1,1\right\} \cdot \mu_{\mathbf{N}, \mathbf{M}}(k, l)\right\} & :=B_{i} \\
i & \in\left\{M_{l-1}+1, \ldots, M_{l}\right\}, \\
\max _{1 \leqslant k \leqslant r}\left\{\max \left\{q_{k}^{-1}-1,1\right\} \cdot \mu_{\mathbf{N}, \mathbf{M}}(k, \infty)\right\} & :=B_{\infty} \quad i>M_{r},
\end{aligned}\right.
$$

and, by again using the assumption that $\|U\| \leqslant 1$,

$$
\begin{array}{rl}
\sum_{j=1}^{N} & \mathbb{E}\left(\left|Z_{j}\right|^{2}\right)=\sum_{j=1}^{N}\left(\tilde{q}_{j}^{-1}-1\right)\left|u_{j i}\right|^{4} \\
& \leqslant \begin{cases}\max _{1 \leqslant k \leqslant r}\left\{\left(q_{k}^{-1}-1\right) \mu_{\mathbf{N}, \mathbf{M}}(k, l)\right\}=: \sigma_{i}^{2} & i \in\left\{M_{l-1}+1, \ldots, M_{l}\right\}, \\
\max _{1 \leqslant k \leqslant r}\left\{\left(q_{k}^{-1}-1\right) \mu_{\mathbf{N}, \mathbf{M}}(k, \infty)\right\}=: \sigma_{\infty}^{2} & i>M_{r} .\end{cases}
\end{array}
$$

Thus, by Bernstein's inequality and (7.58),

$$
\begin{gathered}
\mathbb{P}\left(\left\|P_{\{i\}} U^{*}\left(q_{1}^{-1} P_{\Omega_{1}} \oplus \cdots \oplus q_{r}^{-1} P_{\Omega_{r}}\right) U P_{\{i\}}\right\| \geqslant 1+t\right) \\
\quad \leqslant \mathbb{P}\left(\left|\sum_{j=1}^{N} Z_{j}\right| \geqslant t\right) \leqslant 2 \exp \left(-\frac{t^{2} / 2}{\sigma^{2}+B t / 3}\right),
\end{gathered}
$$




$$
B=\left\{\begin{array}{lll}
\max _{1 \leqslant i \leqslant r} B_{i} & M=M_{r}, \\
\max _{i \in\{1, \ldots, r-1, \infty\}} B_{i} & M>M_{r},
\end{array} \quad \sigma^{2}= \begin{cases}\max _{1 \leqslant i \leqslant r} \sigma_{i}^{2} & M=M_{r}, \\
\max _{i \in\{1, \ldots, r-1, \infty\}} \sigma_{1}^{2} & M>M_{r} .\end{cases}\right.
$$

Applying the union bound yields

$$
\mathbb{P}\left(\max _{i \in\{1, \ldots, M\}}\left\|P_{\{i\}} U^{*}\left(q_{1}^{-1} P_{\Omega_{1}} \oplus \cdots \oplus q_{r}^{-1} P_{\Omega_{r}}\right) U P_{\{i\}}\right\| \geqslant 1+t\right) \leqslant \gamma
$$

whenever (7.56) holds.

7.3. Proofs of Propositions 7.3 and 7.4. The proof of the propositions relies on an idea that originated in a paper by Gross [43], namely, the golfing scheme. The variant we are using here is based on an idea from [3] as well as uneven section techniques from $[47,48]$, see also [42]. However, the informed reader will recognize that the setup here differs substantially from both [43] and [3]. See also [16] for other examples of the use of the golfing scheme. Before we embark on the proof, we state and prove a useful lemma.

LEMMA 7.14. Let $\tilde{X}_{k}$ be independent binary variables taking values 0 and 1 , such that $\tilde{X}_{k}=1$ with probability $P$. Then,

$$
\mathbb{P}\left(\sum_{i=1}^{N} \tilde{X}_{i} \geqslant k\right) \geqslant\left(\frac{N \cdot e}{k}\right)^{-k}\left(\begin{array}{l}
N \\
k
\end{array}\right) P^{k}
$$

Proof. First observe that

$$
\begin{aligned}
\mathbb{P}\left(\sum_{i=1}^{N} \tilde{X}_{i} \geqslant k\right) & =\sum_{i=k}^{N}\left(\begin{array}{c}
N \\
i
\end{array}\right) P^{i}(1-P)^{N-i}=\sum_{i=0}^{N-k}\left(\begin{array}{c}
N \\
i+k
\end{array}\right) P^{i+k}(1-P)^{N-k-i} \\
& =\left(\begin{array}{c}
N \\
k
\end{array}\right) P^{k} \sum_{i=0}^{N-k} \frac{(N-k) ! k !}{(N-i-k) !(i+k) !} P^{i}(1-P)^{N-k-i} \\
& =\left(\begin{array}{c}
N \\
k
\end{array}\right) P^{k} \sum_{i=0}^{N-k}\left(\begin{array}{c}
N-k \\
i
\end{array}\right) P^{i}(1-P)^{N-k-i}\left[\left(\begin{array}{c}
i+k \\
k
\end{array}\right)\right]^{-1} .
\end{aligned}
$$

The result now follows because $\sum_{i=0}^{N-k}\left(\begin{array}{c}N-k \\ i\end{array}\right) P^{i}(1-P)^{N-k-i}=1$ and for $i=0, \ldots, N-k$, we have that

$$
\left(\begin{array}{c}
i+k \\
k
\end{array}\right) \leqslant\left(\frac{(i+k) \cdot e}{k}\right)^{k} \leqslant\left(\frac{N \cdot e}{k}\right)^{k}
$$

where the first inequality follows from Stirling's approximation (see [24], page 1186). 
Proof of Proposition 7.3. We start by mentioning that converting from the Bernoulli sampling model and uniform sampling model has become standard in the literature. In particular, one can do this by showing that the Bernoulli model implies (up to a constant) the uniform sampling model in each of the conditions in Proposition 7.1. This is straightforward and the reader may consult $[17,18,41]$ for details. We therefore consider (without loss of generality) only the multilevel Bernoulli sampling scheme.

Recall that we are using the following Bernoulli sampling model: given $N_{0}=0$, $N_{1}, \ldots, N_{r} \in \mathbb{N}$ we let

$$
\left\{N_{k-1}+1, \ldots, N_{k}\right\} \supset \Omega_{k} \sim \operatorname{Ber}\left(q_{k}\right), \quad q_{k}=\frac{m_{k}}{N_{k}-N_{k-1}} .
$$

Note that we may replace this Bernoulli sampling model with the following equivalent sampling model (see [3]):

$$
\Omega_{k}=\Omega_{k}^{1} \cup \Omega_{k}^{2} \cup \cdots \cup \Omega_{k}^{u}, \quad \Omega_{k}^{j} \sim \operatorname{Ber}\left(q_{k}^{j}\right), \quad 1 \leqslant k \leqslant r,
$$

for some $u \in \mathbb{N}$ with

$$
\left(1-q_{k}^{1}\right)\left(1-q_{k}^{2}\right) \cdots\left(1-q_{k}^{u}\right)=\left(1-q_{k}\right) .
$$

The latter model is the one we use throughout the proof and the specific value of $u$ will be chosen later. Note also that because of overlaps we have

$$
q_{k}^{1}+q_{k}^{2}+\cdots+q_{k}^{u} \geqslant q_{k}, \quad 1 \leqslant k \leqslant r .
$$

The strategy of the proof is to show the validity of (i) and (ii), and the existence of a $\rho \in \operatorname{ran}\left(U^{*}\left(P_{\Omega_{1}} \oplus \cdots \oplus P_{\Omega_{r}}\right)\right)$ that satisfies (iii)-(v) in Proposition 7.1 with probability exceeding $1-\epsilon$, where (iii) is replaced by (7.16), (iv) is replaced by $\left\|P_{M} P_{\Delta}^{\perp} \rho\right\|_{l^{\infty}} \leqslant \frac{1}{2}$ and $L$ in (v) is given by (7.17).

Step I. The construction of $\rho$ : we start by defining $\gamma=\epsilon / 6$ (the reason for this particular choice will become clear later). We also define a number of quantities (and the reason for these choices will become clear later in the proof):

$$
u=8\left\lceil 3 v+\log \left(\gamma^{-1}\right)\right\rceil, \quad v=\left\lceil\log _{2}(8 K M \sqrt{s})\right\rceil,
$$

as well as

$$
\left\{q_{k}^{i}: 1 \leqslant k \leqslant r, 1 \leqslant i \leqslant u\right\}, \quad\left\{\alpha_{i}\right\}_{i=1}^{u}, \quad\left\{\beta_{i}\right\}_{i=1}^{u}
$$

by

$$
q_{k}^{1}=q_{k}^{2}=\frac{1}{4} q_{k}, \quad \tilde{q}_{k}=q_{k}^{3}=\cdots=q_{k}^{u}, \quad q_{k}=\left(N_{k}-N_{k-1}\right) m_{k}^{-1}, \quad 1 \leqslant k \leqslant r,
$$


with

$$
\left(1-q_{k}^{1}\right)\left(1-q_{k}^{2}\right) \cdots\left(1-q_{k}^{u}\right)=\left(1-q_{k}\right)
$$

and

$$
\alpha_{1}=\alpha_{2}=\left(2 \log _{2}^{1 / 2}(4 K M \sqrt{s})\right)^{-1}, \quad \alpha_{i}=1 / 2, \quad 3 \leqslant i \leqslant u,
$$

as well as

$$
\beta_{1}=\beta_{2}=\frac{1}{4}, \quad \beta_{i}=\frac{1}{4} \log _{2}(4 K M \sqrt{s}), \quad 3 \leqslant i \leqslant u .
$$

Consider now the following construction of $\rho$. We define recursively the sequences $\left\{Z_{i}\right\}_{i=0}^{u} \subset \mathcal{H},\left\{Y_{i}\right\}_{i=1}^{u} \subset \mathcal{H}$ and $\left\{\omega_{i}\right\}_{i=0}^{u} \subset \mathbb{N}$ as follows: first let $\omega_{0}=\{0\}, \omega_{1}=\{0,1\}$ and $\omega_{2}=\{0,1,2\}$. Then define recursively, for $i \geqslant 3$, the following:

$$
\begin{aligned}
& \int \omega_{i-1} \cup\{i\} \quad \text { if }\left\|\left(P_{\Delta}-P_{\Delta} U^{*}\left(\frac{1}{q_{1}^{i}} P_{\Omega_{1}^{i}} \oplus \cdots \oplus \frac{1}{q_{r}^{i}} P_{\Omega_{r}^{i}}\right) U P_{\Delta}\right) Z_{i-1}\right\|_{l^{\infty}} \\
& \leqslant \alpha_{i}\left\|P_{\Delta_{k}} Z_{i-1}\right\|_{l^{\infty}}, \\
& \omega_{i}=\left\{\quad \text { and }\left\|P_{M} P_{\Delta}^{\perp} U^{*}\left(\frac{1}{q_{1}^{i}} P_{\Omega_{1}^{i}} \oplus \cdots \oplus \frac{1}{q_{r}^{i}} P_{\Omega_{r}^{i}}\right) U P_{\Delta} Z_{i-1}\right\|_{l^{\infty}}\right. \\
& \leqslant \beta_{i}\left\|Z_{i-1}\right\|_{l^{\infty}}, \\
& \text { otherwise, } \\
& Y_{i}= \begin{cases}\sum_{j \in \omega_{i}} U^{*}\left(\frac{1}{q_{1}^{j}} P_{\Omega_{1}^{j}} \oplus \cdots \oplus \frac{1}{q_{r}^{j}} P_{\Omega_{r}^{j}}\right) U Z_{j-1} \quad \text { if } i \in \omega_{i}, \quad i \geqslant 1, \\
Y_{i-1} & \text { otherwise, }\end{cases} \\
& Z_{i}=\left\{\begin{array}{ll}
\operatorname{sgn}\left(x_{0}\right)-P_{\Delta} Y_{i} & \text { if } i \in \omega_{i}, \\
Z_{i-1} & \text { otherwise, }
\end{array} \quad i \geqslant 1, Z_{0}=\operatorname{sgn}\left(x_{0}\right) .\right.
\end{aligned}
$$

Now, let $\left\{A_{i}\right\}_{i=1}^{2}$ and $\left\{B_{i}\right\}_{i=1}^{5}$ denote the following events

$$
\begin{array}{c|c}
A_{i}: \quad & \left\|\left(P_{\Delta}-U^{*}\left(\frac{1}{q_{1}^{i}} P_{\Omega_{1}^{i}} \oplus \cdots \oplus \frac{1}{q_{r}^{i}} P_{\Omega_{r}^{i}}\right) U P_{\Delta}\right) Z_{i-1}\right\|_{l^{\infty}} \leqslant \alpha_{i}\left\|Z_{i-1}\right\|_{l^{\infty}}, \\
i & =1,2, \\
B_{i}: \quad & \left\|P_{M} P_{\Delta}^{\perp} U^{*}\left(\frac{1}{q_{1}^{i}} P_{\Omega_{1}^{i}} \oplus \cdots \oplus \frac{1}{q_{r}^{i}} P_{\Omega_{r}^{i}}\right) U P_{\Delta} Z_{i-1}\right\|_{l^{\infty}} \leqslant \beta_{i}\left\|Z_{i-1}\right\|_{l^{\infty}}, \\
i & =1,2,
\end{array}
$$




$$
\begin{aligned}
B_{3}: & \left\|P_{\Delta} U^{*}\left(\frac{1}{q_{1}} P_{\Omega_{1}} \oplus \cdots \oplus \frac{1}{q_{r}} P_{\Omega_{r}}\right) U P_{\Delta}-P_{\Delta}\right\| \leqslant 1 / 4, \\
& \max _{i \in \Delta^{c} \cap\{1, \ldots, M\}}\left\|\left(q_{1}^{-1 / 2} P_{\Omega_{1}} \oplus \cdots \oplus q_{r}^{-1 / 2} P_{\Omega_{r}}\right) U e_{i}\right\| \leqslant \sqrt{5 / 4} \\
B_{4}: & \left|\omega_{u}\right| \geqslant v, \\
B_{5}: & \left(\cap_{i=1}^{2} A_{i}\right) \cap\left(\cap_{i=1}^{4} B_{i}\right) .
\end{aligned}
$$

Also, let $\tau(j)$ denote the $j$ th element in $\omega_{u}$ (for example, $\tau(0)=0, \tau(1)=1$, $\tau(2)=2$ ) and finally define $\rho$ by

$$
\rho= \begin{cases}Y_{\tau(v)} & \text { if } B_{5} \text { occurs } \\ 0 & \text { otherwise. }\end{cases}
$$

Note that, clearly, $\rho \in \operatorname{ran}\left(U^{*} P_{\Omega}\right)$, and we just need to show that when the event $B_{5}$ occurs, then (i) $-(\mathrm{v})$ in Proposition 7.1 will follow.

Step II. $B_{5} \Rightarrow(i)$, (ii). To see that the assertion is true, note that if $B_{5}$ occurs then $B_{3}$ occurs, which immediately (i) and (ii).

Step III. $B_{5} \Rightarrow$ (iii), (iv). To show the assertion, we start by making the following observations: by the construction of $Z_{\tau(i)}$ and the fact that $Z_{0}=\operatorname{sgn}\left(x_{0}\right)$, it follows that

$$
\begin{aligned}
Z_{\tau(i)}= & Z_{0}-\left(P_{\Delta} U^{*}\left(\frac{1}{q_{1}^{\tau(1)}} P_{\Omega_{1}^{\tau(1)}} \oplus \cdots \oplus \frac{1}{q_{r}^{\tau(1)}} P_{\Omega_{r}^{\tau(i)}}\right) U P_{\Delta}\right) Z_{0} \\
& +\cdots+\left(P_{\Delta} U^{*}\left(\frac{1}{q_{1}^{\tau(i)}} P_{\Omega_{1}^{\tau(i)}} \oplus \cdots \oplus \frac{1}{q_{r}^{\tau(i)}} P_{\Omega_{r}^{\tau(i)}}\right) U P_{\Delta}\right) Z_{\tau(i-1)} \\
= & Z_{\tau(i-1)}-\left(P_{\Delta} U^{*}\left(\frac{1}{q_{1}^{\tau(i)}} P_{\Omega_{1}^{\tau(i)}} \oplus \cdots \oplus \frac{1}{q_{r}^{\tau(i)}} P_{\Omega_{r}^{\tau(i)}}\right) U P_{\Delta}\right) Z_{\tau(i-1)} \\
& i \leqslant\left|\omega_{u}\right|,
\end{aligned}
$$

so we immediately get that

$$
Z_{\tau(i)}=\left(P_{\Delta}-P_{\Delta} U^{*}\left(\frac{1}{q_{1}^{\tau(i)}} P_{\Omega_{1}^{\tau(i)}} \oplus \cdots \oplus \frac{1}{q_{r}^{\tau(i)}} P_{\Omega_{r}^{\tau(i)}}\right) U P_{\Delta}\right) Z_{\tau(i-1)}, \quad i \leqslant\left|\omega_{u}\right| .
$$

Hence, if the event $B_{5}$ occurs, we have, by the choices in (7.64) and (7.65)

$$
\left\|\rho-\operatorname{sgn}\left(x_{0}\right)\right\|=\left\|Z_{\tau(v)}\right\| \leqslant \sqrt{s}\left\|Z_{\tau(v)}\right\|_{l^{\infty}} \leqslant \sqrt{s} \prod_{i=1}^{v} \alpha_{\tau(i)} \leqslant \frac{\sqrt{s}}{2^{v}} \leqslant \frac{1}{8 K},
$$


since we have chosen $v=\left\lceil\log _{2}(8 K M \sqrt{s})\right\rceil$. Also,

$$
\begin{aligned}
\left\|P_{M} P_{\Delta}^{\perp} \rho\right\|_{l^{\infty}} & \leqslant \sum_{i=1}^{v}\left\|P_{M} P_{\Delta}^{\perp} U^{*}\left(\frac{1}{q_{1}^{\tau(i)}} P_{\Omega_{1}^{\tau(i)}} \oplus \cdots \oplus \frac{1}{q_{r}^{\tau(i)}} P_{\Omega_{r}^{\tau(i)}}\right) U P_{\Delta} Z_{\tau(i-1)}\right\|_{l^{\infty}} \\
& \leqslant \sum_{i=1}^{v} \beta_{\tau(i)}\left\|Z_{\tau(i-1)}\right\|_{l^{\infty}} \leqslant \sum_{i=1}^{v} \beta_{\tau(i)} \prod_{j=1}^{i-1} \alpha_{\tau(j)} \\
& \leqslant \frac{1}{4}\left(1+\frac{1}{2 \log _{2}^{1 / 2}(a)}+\frac{\log _{2}(a)}{2^{3} \log _{2}(a)}+\cdots+\frac{1}{2^{v-1}}\right) \leqslant \frac{1}{2}, \quad \text { (7.69) }
\end{aligned}
$$

where $a=4 K M \sqrt{s}$. In particular, (7.68) and (7.69) imply (iii) and (iv) in Proposition 7.1.

Step IV. $B_{5} \Rightarrow(v)$. To show that, note that we may write the already constructed $\rho$ as $\rho=U^{*} P_{\Omega} w$ where

$$
w=\sum_{i=1}^{v} w_{i}, \quad w_{i}=\left(\frac{1}{q_{1}^{\tau(i)}} P_{\Omega_{1}} \oplus \cdots \oplus \frac{1}{q_{r}^{\tau(i)}} P_{\Omega_{r}}\right) U P_{\Delta} Z_{\tau(i-1)} .
$$

To estimate $\|w\|$ we simply compute

$$
\begin{aligned}
\left\|w_{i}\right\|^{2}= & \left\langle\left(\frac{1}{q_{1}^{\tau(i)}} P_{\Omega_{1}^{\tau(i)}} \oplus \cdots \oplus \frac{1}{q_{r}^{\tau(i)}} P_{\Omega_{r}^{\tau(i)}}\right) U P_{\Delta} Z_{\tau(i-1)},\right. \\
& \left.\left(\frac{1}{q_{1}^{\tau(i)}} P_{\Omega_{1}^{\tau(i)}} \oplus \cdots \oplus \frac{1}{q_{r}^{\tau(i)}} P_{\Omega_{r}^{\tau(i)}}\right) U P_{\Delta} Z_{\tau(i-1)}\right\rangle \\
= & \sum_{k=1}^{r}\left(\frac{1}{q_{k}^{\tau(i)}}\right)^{2}\left\|P_{\Omega_{k}^{\tau(i)}} U Z_{\tau(i-1)}\right\|^{2}
\end{aligned}
$$

and then use the assumption that the event $B_{5}$ holds to deduce that

$$
\begin{aligned}
& \sum_{k=1}^{r}\left(\frac{1}{q_{k}^{\tau(i)}}\right)^{2}\left\|P_{\Omega_{k}^{\tau(i)}} U Z_{\tau(i-1)}\right\|^{2} \\
& \quad \leqslant \max _{1 \leqslant k \leqslant r}\left\{\frac{1}{q_{k}^{\tau(i)}}\right\}\left\langle\sum_{k=1}^{r} \frac{1}{q_{k}^{\tau(i)}} P_{\Delta} U^{*} P_{\Omega_{k}^{\tau(i)}} U Z_{\tau(i-1)}, Z_{\tau(i-1)}\right\rangle \\
& \quad=\max _{1 \leqslant k \leqslant r}\left\{\frac{1}{q_{k}^{\tau(i)}}\right\}\left\langle\left(\sum_{k=1}^{r} \frac{1}{q_{k}^{\tau(i)}} P_{\Delta} U^{*} P_{\Omega_{k}^{\tau(i)}} U-P_{\Delta}\right) Z_{\tau(i-1)}, Z_{\tau(i-1)}\right\rangle
\end{aligned}
$$




$$
\begin{aligned}
& +\left\|Z_{\tau(i-1)}\right\|^{2} \\
\leqslant & \max _{1 \leqslant k \leqslant r}\left\{\frac{1}{q_{k}^{\tau(i)}}\right\}\left(\left\|Z_{\tau(i-1)}\right\|\left\|Z_{\tau(i)}\right\|+\left\|Z_{\tau(i-1)}\right\|^{2}\right) \\
\leqslant & \max _{1 \leqslant k \leqslant r}\left\{\frac{1}{q_{k}^{\tau(i)}}\right\} s\left(\left\|Z_{\tau(i-1)}\right\|_{l^{\infty}}\left\|Z_{\tau(i)}\right\|_{l^{\infty}}+\left\|Z_{\tau(i-1)}\right\|_{l^{\infty}}^{2}\right) \\
\leqslant & \max _{1 \leqslant k \leqslant r}\left\{\frac{1}{q_{k}^{\tau(i)}}\right\} s\left(\alpha_{i}+1\right)\left(\prod_{j=1}^{i-1} \alpha_{j}\right)^{2},
\end{aligned}
$$

where the last inequality follows from the assumption that the event $B_{5}$ holds. Hence

$$
\|w\| \leqslant \sqrt{s} \sum_{i=1}^{v}\left(\max _{1 \leqslant k \leqslant r}\left\{\frac{1}{\sqrt{q_{k}^{\tau(i)}}}\right\} \sqrt{\alpha_{i}+1} \prod_{j=1}^{i-1} \alpha_{j}\right) .
$$

Note that, due to the fact that $q_{k}^{1}+\cdots+q_{k}^{u} \geqslant q_{k}$, we have that

$$
\tilde{q}_{k} \geqslant \frac{m_{k}}{2\left(N_{k}-N_{k-1}\right)} \frac{1}{8\left\lceil\log \left(\gamma^{-1}\right)+3\left\lceil\log _{2}(8 K M \sqrt{s})\right\rceil\right\rceil-2} .
$$

This gives, in combination with the chosen values of $\left\{\alpha_{j}\right\}$ and (7.70) that

$$
\begin{aligned}
& \|w\| \leqslant 2 \sqrt{s} \max _{1 \leqslant k \leqslant r} \sqrt{\frac{N_{k}-N_{k-1}}{m_{k}}}\left(1+\frac{1}{2 \log _{2}^{1 / 2}(4 K M \sqrt{s})}\right)^{3 / 2} \\
& +\sqrt{s} \max _{1 \leqslant k \leqslant r} \sqrt{\frac{N_{k}-N_{k-1}}{m_{k}}} \cdot \frac{\sqrt{3}}{2} \\
& \times \frac{\sqrt{8\left\lceil\log \left(\gamma^{-1}\right)+3\left\lceil\log _{2}(8 K M \sqrt{s})\right\rceil\right\rceil-2}}{\log _{2}(4 K M \sqrt{s})} \cdot \sum_{i=3}^{v} \frac{1}{2^{i-3}} \\
& \leqslant 2 \sqrt{s} \max _{1 \leqslant k \leqslant r} \sqrt{\frac{N_{k}-N_{k-1}}{m_{k}}} \\
& \times\left(\left(\frac{3}{2}\right)^{3 / 2}+\sqrt{\frac{6}{\log _{2}(4 K M \sqrt{s})}} \sqrt{1+\frac{\log _{2}\left(\gamma^{-1}\right)+6}{\log _{2}(4 K M \sqrt{s})}}\right)
\end{aligned}
$$




$$
\begin{aligned}
\leqslant & \sqrt{s} \max _{1 \leqslant k \leqslant r} \sqrt{\frac{N_{k}-N_{k-1}}{m_{k}}} \\
& \times\left(\frac{3 \sqrt{3}}{\sqrt{2}}+\frac{2 \sqrt{6}}{\sqrt{\log _{2}(4 K M \sqrt{s})}} \sqrt{1+\frac{\log _{2}\left(\gamma^{-1}+6\right.}{\log _{2}(4 K M \sqrt{s})}}\right) .
\end{aligned}
$$

Step $V$. The weak balancing property, (7.14) and (7.15) $\Rightarrow \mathbb{P}\left(A_{1}^{c} \cup A_{2}^{c} \cup B_{1}^{c} \cup\right.$ $\left.B_{2}^{c} \cup B_{3}^{c}\right) \leqslant 5 \gamma$. To see this, note that by Proposition 7.12 we immediately get (recall that $q_{k}^{1}=q_{k}^{2}=1 / 4 q_{k}$ ) that $\mathbb{P}\left(A_{1}^{c}\right) \leqslant \gamma$ and $\mathbb{P}\left(A_{2}^{c}\right) \leqslant \gamma$ as long as the weak balancing property and

$$
\begin{aligned}
& 1 \gtrsim \Lambda \cdot\left(\log \left(s \gamma^{-1}\right)+1\right) \cdot \log (\sqrt{s} K M), \\
& 1 \gtrsim \Upsilon \cdot\left(\log \left(s \gamma^{-1}\right)+1\right) \cdot \log (\sqrt{s} K M),
\end{aligned}
$$

are satisfied, where $K=\max _{1 \leqslant k \leqslant r}\left(N_{k}-N_{k-1}\right) / m_{k}$,

$$
\begin{gathered}
\Lambda=\max _{1 \leqslant k \leqslant r}\left\{\frac{N_{k}-N_{k-1}}{m_{k}} \cdot\left(\sum_{l=1}^{r} \kappa_{\mathbf{N}, \mathbf{M}}(k, l)\right)\right\}, \\
\Upsilon=\max _{1 \leqslant l \leqslant r} \sum_{k=1}^{r}\left(\frac{N_{k}-N_{k-1}}{m_{k}}-1\right) \cdot \mu_{\mathbf{N}, \mathbf{M}}(k, l) \cdot \tilde{s}_{k},
\end{gathered}
$$

and where $\tilde{s}_{1}+\cdots+\tilde{s}_{r} \leqslant s_{1}+\cdots+s_{r}$ and $\tilde{s}_{k} \leqslant S_{k}\left(s_{1}, \ldots, s_{r}\right)$. However, clearly, (7.14) and (7.15) imply (7.72). Also, Proposition 7.11 yields that $\mathbb{P}\left(B_{1}^{c}\right) \leqslant \gamma$ and $\mathbb{P}\left(B_{2}^{c}\right) \leqslant \gamma$ as long as the weak balancing property and

$$
1 \gtrsim \Lambda \cdot \log \left(\frac{4}{\gamma}(M-s)\right), \quad 1 \gtrsim \Upsilon \cdot \log \left(\frac{4}{\gamma}(M-s)\right),
$$

are satisfied. However, again, (7.14) and (7.15) imply (7.75). Finally, it remains to bound $\mathbb{P}\left(B_{3}^{c}\right)$. First note that by Theorem 7.8 , we may deduce that

$$
\mathbb{P}\left(\left\|P_{\Delta} U^{*}\left(\frac{1}{q_{1}} P_{\Omega_{1}} \oplus \cdots \oplus \frac{1}{q_{r}} P_{\Omega_{r}}\right) U P_{\Delta}-P_{\Delta}\right\|>1 / 4,\right) \leqslant \gamma / 2,
$$

when the weak balancing property and

$$
1 \gtrsim \Lambda \cdot\left(\log \left(\gamma^{-1} s\right)+1\right)
$$

holds and (7.14) implies (7.76).

For the second part of $B_{3}$, we may deduce from Proposition 7.13 that

$$
\mathbb{P}\left(\max _{i \in \Delta^{c} \cap\{1, \ldots, M\}}\left\|\left(q_{1}^{-1 / 2} P_{\Omega_{1}} \oplus \cdots \oplus q_{r}^{-1 / 2} P_{\Omega_{r}}\right) U e_{i}\right\|>\sqrt{5 / 4}\right) \leqslant \frac{\gamma}{2},
$$


whenever

$$
1 \gtrsim \log \left(\frac{2 M}{\gamma}\right) \cdot \max _{1 \leqslant k \leqslant r}\left\{\left(\frac{N_{k}-N_{k-1}}{m_{k}}-1\right) \cdot \mu_{\mathbf{N}, \mathbf{M}}(k, l)\right\}, \quad l=1, \ldots, r
$$

which is true whenever (7.14) holds. Indeed, recalling the definition of $\kappa_{\mathbf{N}, \mathbf{M}}(k, j)$ and $\Theta$ in Definition 7.2, observe that

$$
\max _{\eta \in \Theta,\|\eta\|_{\infty}=1} \sum_{l=1}^{r}\left\|P_{N_{k}}^{N_{k-1}} U P_{M_{l}}^{M_{l-1}} \eta\right\|_{\infty} \geqslant \max _{\eta \in \Theta,\|\eta\|_{\infty}=1}\left\|P_{N_{k}}^{N_{k-1}} U \eta\right\|_{\infty} \geqslant \sqrt{\mu\left(P_{N_{k}}^{N_{k-1}} U P_{M_{l}}^{M_{l-1}}\right)}
$$

for each $l=1, \ldots, r$ which implies that $\sum_{j=1}^{r} \kappa_{\mathbf{N}, \mathbf{M}}(k, j) \geqslant \mu_{\mathbf{N}, \mathbf{M}}(k, l)$, for $l=1, \ldots, r$. Consequently, (7.77) follows from (7.14). Thus, $\mathbb{P}\left(B_{3}^{c}\right) \leqslant \gamma$.

Step VI. The weak balancing property, (7.14) and (7.15) $\Rightarrow \mathbb{P}\left(B_{4}^{c}\right) \leqslant \gamma$. To see this, define the random variables $X_{1}, \ldots, X_{u-2}$ by

$$
X_{j}= \begin{cases}0 & \omega_{j+2} \neq \omega_{j+1} \\ 1 & \omega_{j+2}=\omega_{j+1}\end{cases}
$$

We immediately observe that

$$
\mathbb{P}\left(B_{4}^{c}\right)=\mathbb{P}\left(\left|\omega_{u}\right|<v\right)=\mathbb{P}\left(X_{1}+\cdots+X_{u-2}>u-v\right) .
$$

However, the random variables $X_{1}, \ldots, X_{u-2}$ are not independent, and we therefore cannot directly apply the standard Chernoff bound. In particular, we must adapt the setup slightly. Note that

$$
\begin{aligned}
\mathbb{P} & \left(X_{1}+\cdots+X_{u-2}>u-v\right) \\
\leqslant & \sum_{l=1}^{\substack{u-2 \\
u-v}} \mathbb{P}\left(X_{\pi(l)_{1}}=1, X_{\pi(l)_{2}}=1, \ldots, X_{\pi(l)_{u-v}}=1\right) \\
= & \sum_{l=1}^{\substack{u-2 \\
u-v}} \mathbb{P}\left(X_{\pi(l)_{u-v}}=1 \mid X_{\pi(l)_{1}}=1, \ldots, X_{\pi(l)_{u-v-1}}=1\right) \\
& \times \mathbb{P}\left(X_{\pi(l)_{1}}=1, \ldots, X_{\pi(l)_{u-v-1}}=1\right) \\
= & \sum_{l=1}^{\substack{u-2 \\
u-v}} \mathbb{P}\left(X_{\pi(l)_{u-v}}=1 \mid X_{\pi(l)_{1}}=1, \ldots, X_{\pi(l)_{u-v-1}}=1\right) \\
& \times \mathbb{P}\left(X_{\pi(l)_{u-v-1}}=1 \mid X_{\pi(l)_{1}}=1, \ldots, X_{\pi(l)_{u-v-2}}=1\right) \cdots \mathbb{P}\left(X_{\pi(l)_{1}}=1\right)
\end{aligned}
$$


where $\pi:\left\{1, \ldots,\left(\begin{array}{l}u-2 \\ u-v\end{array}\right)\right\} \rightarrow \mathbb{N}^{u-v}$ ranges over all $\left(\begin{array}{l}u-2 \\ u-v\end{array}\right)$ ordered subsets of $\{1, \ldots, u-2\}$ of size $u-v$. Thus, if we can provide a bound $P$ such that

$$
\begin{aligned}
& P \geqslant \mathbb{P}\left(X_{\pi(l)_{u-v-j}}=1 \mid X_{\pi(l)_{1}}=1, \ldots, X_{\pi(l)_{u-v-(j+1)}}=1\right), \\
& P \geqslant \mathbb{P}\left(X_{\pi(l)_{1}}=1\right) \\
& \quad l=1, \ldots,\left(\begin{array}{c}
u-2 \\
u-v
\end{array}\right), \quad j=0, \ldots, u-v-2,
\end{aligned}
$$

then, by (7.81),

$$
\mathbb{P}\left(X_{1}+\cdots+X_{u-2}>u-v\right) \leqslant\left(\begin{array}{l}
u-2 \\
u-v
\end{array}\right) P^{u-v} .
$$

We continue assuming that (7.82) is true, and then return to this inequality below.

Let $\left\{\tilde{X}_{k}\right\}_{k=1}^{u-2}$ be independent binary variables taking values 0 and 1 , such that $\tilde{X}_{k}=1$ with probability $P$. Then, by Lemma $7.14,(7.83)$ and (7.80) it follows that

$$
\mathbb{P}\left(B_{4}^{c}\right) \leqslant \mathbb{P}\left(\tilde{X}_{1}+\cdots+\tilde{X}_{u-2} \geqslant u-v\right)\left(\frac{(u-2) \cdot e}{u-v}\right)^{u-v} .
$$

Then, by the standard Chernoff bound [65, Theorem 2.1, equation 2], it follows that, for $t>0$,

$$
\mathbb{P}\left(\tilde{X}_{1}+\cdots+\tilde{X}_{u-2} \geqslant(u-2)(t+P)\right) \leqslant e^{-2(u-2) t^{2}} .
$$

Hence, if we let $t=(u-v) /(u-2)-P$, it follows from (7.84) and (7.85) that

$$
\mathbb{P}\left(B_{4}^{c}\right) \leqslant e^{-2(u-2) t^{2}+(u-v)(\log ((u-2) /(u-v))+1)} \leqslant e^{-2(u-2) t^{2}+u-2} .
$$

Thus, by choosing $P=1 / 4$ we get that $\mathbb{P}\left(B_{4}^{c}\right) \leqslant \gamma$ whenever $u \geqslant x$ and $x$ is the largest root satisfying

$$
(x-u)\left(\frac{x-v}{u-2}-\frac{1}{4}\right)-\log \left(\gamma^{-1 / 2}\right)-\frac{x-2}{2}=0,
$$

and this yields $u \geqslant 8\left\lceil 3 v+\log \left(\gamma^{-1 / 2}\right)\right\rceil$ which is satisfied by the choice of $u$ in (7.62). Thus, we would have been done with Step VI if we could verify (7.82) with $P=1 / 4$, and this is the theme in the following claim.

Claim. The weak balancing property, (7.14) and (7.15) $\Rightarrow$ (7.82) with $P=1 / 4$. To prove the claim we first observe that $X_{j}=0$ when

$$
\left\|\left(P_{\Delta}-P_{\Delta} U^{*}\left(\frac{1}{q_{1}^{i}} P_{\Omega_{1}^{i}} \oplus \cdots \oplus \frac{1}{q_{r}^{i}} P_{\Omega_{r}^{i}}\right) U P_{\Delta}\right) Z_{i-1}\right\|_{l^{\infty}} \leqslant \frac{1}{2}\left\|Z_{i-1}\right\|_{l^{\infty}}
$$




$$
\begin{gathered}
\| P_{M} P_{\Delta}^{\perp} U^{*} \\
\left(\frac{1}{q_{1}^{i}} P_{\Omega_{1}^{i}} \oplus \cdots \oplus \frac{1}{q_{r}^{i}} P_{\Omega_{r}^{i}}\right) U P_{\Delta} Z_{i-1}\left\|_{l^{\infty}} \leqslant \frac{1}{4} \log _{2}(4 K M \sqrt{s})\right\| Z_{i-1} \|_{l^{\infty}}, \\
i=j+2,
\end{gathered}
$$

where we recall from (7.63) that

$$
q_{k}^{3}=q_{k}^{4}=\cdots=q_{k}^{u}=\tilde{q}_{k}, \quad 1 \leqslant k \leqslant r .
$$

Thus, by choosing $\gamma=1 / 8$ in (7.48) in Proposition 7.12 and $\gamma=1 / 8$ in (i) in Proposition 7.11 , it follows that $\frac{1}{4} \geqslant \mathbb{P}\left(X_{j}=1\right)$, for $j=1, \ldots, u-2$, when the weak balancing property is satisfied and

$$
\begin{aligned}
& (\log (8 s)+1)^{-1} \gtrsim \tilde{q}_{k}^{-1} \cdot \sum_{l=1}^{r} \kappa_{\mathbf{N}, \mathbf{M}}(k, l), \quad 1 \leqslant k \leqslant r \\
& (\log (8 s)+1)^{-1} \gtrsim\left(\sum_{k=1}^{r}\left(\tilde{q}_{k}^{-1}-1\right) \cdot \mu_{\mathbf{N}, \mathbf{M}}(k, l) \cdot \tilde{s}_{k}\right), \quad 1 \leqslant l \leqslant r,
\end{aligned}
$$

as well as

$$
\begin{aligned}
& \frac{\log _{2}(4 K M \sqrt{s})}{\log (32(M-s))} \gtrsim \tilde{q}_{k}^{-1} \cdot \sum_{l=1}^{r} \kappa_{\mathbf{N}, \mathbf{M}}(k, l), \quad 1 \leqslant k \leqslant r \\
& \frac{\log _{2}(4 K M \sqrt{s})}{\log (32(M-s))} \gtrsim\left(\sum_{k=1}^{r}\left(\tilde{q}_{k}^{-1}-1\right) \cdot \mu_{\mathbf{N}, \mathbf{M}}(k, l) \cdot \tilde{s}_{k}\right), \quad 1 \leqslant l \leqslant r,
\end{aligned}
$$

with $K=\max _{1 \leqslant k \leqslant r}\left(N_{k}-N_{k-1}\right) / m_{k}$. Thus, to prove the claim we must demonstrate that (7.14) and (7.15) $\Rightarrow$ (7.86), (7.87), (7.88) and (7.89). We split this into two stages:

Stage 1 . (7.15) $\Rightarrow$ (7.89) and (7.87). To show the assertion we must demonstrate that if, for $1 \leqslant k \leqslant r$,

$$
m_{k} \gtrsim\left(\log \left(s \epsilon^{-1}\right)+1\right) \cdot \hat{m}_{k} \cdot \log (K M \sqrt{s}),
$$

where $\hat{m}_{k}$ satisfies

$$
1 \gtrsim \sum_{k=1}^{r}\left(\frac{N_{k}-N_{k-1}}{\hat{m}_{k}}-1\right) \cdot \mu_{\mathbf{N}, \mathbf{M}}(k, l) \cdot \tilde{s}_{k}, \quad l=1, \ldots, r,
$$

we get (7.89) and (7.87). To see this, note that by (7.61) we have that

$$
q_{k}^{1}+q_{k}^{2}+(u-2) \tilde{q}_{k} \geqslant q_{k}, \quad 1 \leqslant k \leqslant r,
$$


so since $q_{k}^{1}=q_{k}^{2}=\frac{1}{4} q_{k}$, and by (7.92), (7.90) and the choice of $u$ in (7.62), it follows that

$$
\begin{aligned}
2\left(8 \left(\left\lceil\log \left(\gamma^{-1}\right)+\right.\right.\right. & \left.\left.\left.3\left\lceil\log _{2}(8 K M \sqrt{s})\right\rceil\right\rceil\right)-2\right) \tilde{q}_{k} \geqslant q_{k}=\frac{m_{k}}{N_{k}-N_{k-1}} \\
& \geqslant C \frac{\hat{m}_{k}}{N_{k}-N_{k-1}}\left(\log \left(s \epsilon^{-1}\right)+1\right) \log (K M \sqrt{s}) \\
& \geqslant C \frac{\hat{m}_{k}}{N_{k}-N_{k-1}}(\log (s)+1)\left(\log (K M \sqrt{s})+\log \left(\epsilon^{-1}\right)\right),
\end{aligned}
$$

for some constant $C$ (recall that we have assumed that $\log (s) \geqslant 1$ ). And this gives (by recalling that $\gamma=\epsilon / 6)$ that $\tilde{q}_{k} \geqslant \hat{C}\left(\hat{m}_{k} /\left(N_{k}-N_{k-1}\right)\right)(\log (s)+1)$, for some constant $\hat{C}$. Thus, (7.15) implies that for $1 \leqslant l \leqslant r$,

$$
\begin{aligned}
1 & \gtrsim(\log (s)+1)\left(\sum_{k=1}^{r}\left(\frac{N_{k}-N_{k-1}}{m_{k}(\log (s)+1)}-\frac{1}{\log (s)+1}\right) \cdot \mu_{\mathbf{N}, \mathbf{M}}(k, l) \cdot \tilde{s}_{k}\right) \\
& \gtrsim(\log (s)+1)\left(\sum_{k=1}^{r}\left(\tilde{q}_{k}^{-1}-1\right) \cdot \mu_{\mathbf{N}, \mathbf{M}}(k, l) \cdot \tilde{s}_{k}\right),
\end{aligned}
$$

and this implies (7.89) and (7.87), given an appropriate choice of the constant $C$.

Stage 2. (7.14) $\Rightarrow$ (7.88) and (7.86). To show the assertion we must demonstrate that if, for $1 \leqslant k \leqslant r$,

$$
1 \gtrsim\left(\log \left(s \epsilon^{-1}\right)+1\right) \cdot \frac{N_{k}-N_{k-1}}{m_{k}} \cdot\left(\sum_{l=1}^{r} \kappa_{\mathbf{N}, \mathbf{M}}(k, l)\right) \cdot \log (K M \sqrt{s}),
$$

we obtain (7.88) and (7.86). To see this, note that by arguing as above via the fact that $q_{k}^{1}=q_{k}^{2}=\frac{1}{4} q_{k}$, and by (7.92), (7.93) and the choice of $u$ in (7.62) we have that

$$
\begin{aligned}
2\left(8\left(\left\lceil\log \left(\gamma^{-1}\right)+3\left\lceil\log _{2}(8 K M \sqrt{s})\right\rceil\right\rceil\right)-2\right) \tilde{q}_{k} \geqslant q_{k}=\frac{m_{k}}{N_{k}-N_{k-1}} \\
\quad \geqslant C \cdot\left(\sum_{l=1}^{r} \kappa_{\mathbf{N}, \mathbf{M}}(k, l)\right) \cdot\left(\log \left(s \epsilon^{-1}\right)+1\right) \cdot \log (K M \sqrt{s}) \\
\quad \geqslant C \cdot\left(\sum_{l=1}^{r} \kappa_{\mathbf{N}, \mathbf{M}}(k, l)\right) \cdot(\log (s)+1)\left(\log \left(\epsilon^{-1}\right)+\log (K M \sqrt{s})\right),
\end{aligned}
$$

for some constant $C$. Thus, we have that for some appropriately chosen constant $\hat{C}, \tilde{q}_{k} \geqslant \hat{C} \cdot(\log (s)+1) \cdot \sum_{l=1}^{r} \kappa_{\mathbf{N}, \mathbf{M}}(k, l)$. So, (7.88) and (7.86) holds given an appropriately chosen $C$. This yields the last puzzle of the proof, and we are done. 
Proof of Proposition 7.4. The proof is very close to the proof of Proposition 7.3 and we simply point out the differences. The strategy of the proof is to show the validity of (i) and (ii), and the existence of a $\rho \in \operatorname{ran}\left(U^{*}\left(P_{\Omega_{1}} \oplus \cdots \oplus P_{\Omega_{r}}\right)\right)$ that satisfies (iii)-(v) in Proposition 7.1 with probability exceeding $1-\epsilon$.

Step I. The construction of $\rho$ : the construction is almost identical to the construction in the proof of Proposition 7.3, except that

$$
\begin{gathered}
u=8\left\lceil\log \left(\gamma^{-1}\right)+3 v\right\rceil, \quad v=\left\lceil\log _{2}(8 K \tilde{M} \sqrt{s})\right\rceil, \\
\alpha_{1}=\alpha_{2}=\left(2 \log _{2}^{1 / 2}(4 K \tilde{M} \sqrt{s})\right)^{-1}, \quad \alpha_{i}=1 / 2, \quad 3 \leqslant i \leqslant u,
\end{gathered}
$$

as well as

$$
\beta_{1}=\beta_{2}=\frac{1}{4}, \quad \beta_{i}=\frac{1}{4} \log _{2}(4 K \tilde{M} \sqrt{s}), \quad 3 \leqslant i \leqslant u,
$$

and (7.66) gets changed to

$$
\omega_{i}=\left\{\begin{array}{cc}
\omega_{i-1} \cup\{i\} \quad \text { if } \| & \left(P_{\Delta}-P_{\Delta} U^{*}\left(\frac{1}{q_{1}^{i}} P_{\Omega_{1}^{i}} \oplus \cdots \oplus \frac{1}{q_{r}^{i}} P_{\Omega_{r}^{i}}\right) U P_{\Delta}\right) Z_{i-1} \|_{l^{\infty}} \\
& \leqslant \alpha_{i}\left\|P_{\Delta_{k}} Z_{i-1}\right\|_{l^{\infty}}, \\
& \text { and }\left\|P_{\Delta}^{\perp} U^{*}\left(\frac{1}{q_{1}^{i}} P_{\Omega_{1}^{i}} \oplus \cdots \oplus \frac{1}{q_{r}^{i}} P_{\Omega_{r}^{i}}\right) U P_{\Delta} Z_{i-1}\right\|_{l^{\infty}} \\
& \leqslant \beta_{i}\left\|Z_{i-1}\right\|_{l^{\infty}},
\end{array}\right.
$$

the events $B_{i}, i=1,2$ in (7.67) get replaced by

$\widetilde{B}_{i}: \quad\left\|P_{\Delta}^{\perp} U^{*}\left(\frac{1}{q_{1}^{i}} P_{\Omega_{1}^{i}} \oplus \cdots \oplus \frac{1}{q_{r}^{i}} P_{\Omega_{r}^{i}}\right) U P_{\Delta} Z_{i-1}\right\|_{l^{\infty}} \leqslant \beta_{i}\left\|Z_{i-1}\right\|_{l^{\infty}}, \quad i=1,2$,

and the second part of $B_{3}$ becomes

$$
\max _{i \in \Delta^{c}}\left\|\left(q_{1}^{-1 / 2} P_{\Omega_{1}} \oplus \cdots \oplus q_{r}^{-1 / 2} P_{\Omega_{r}}\right) U e_{i}\right\| \leqslant \sqrt{5 / 4}
$$

Step II. $B_{5} \Rightarrow(i),(i i)$. This step is identical to Step II in the proof of Proposition 7.3. 
Step III. $B_{5} \Rightarrow$ (iii), (iv). Equation (7.69) gets changed to

$$
\begin{aligned}
\left\|P_{\Delta}^{\perp} \rho\right\|_{l^{\infty}} \leqslant & \sum_{i=1}^{v}\left\|P_{\Delta}^{\perp} U^{*}\left(\frac{1}{q_{1}^{\tau(i)}} P_{\Omega_{1}^{\tau(i)}} \oplus \cdots \oplus \frac{1}{q_{r}^{\tau(i)}} P_{\Omega_{r}^{\tau(i)}}\right) U P_{\Delta} Z_{\tau(i-1)}\right\|_{l^{\infty}} \\
\leqslant & \sum_{i=1}^{v} \beta_{\tau(i)}\left\|Z_{\tau(i-1)}\right\|_{l^{\infty}} \leqslant \sum_{i=1}^{v} \beta_{\tau(i)} \prod_{j=1}^{i-1} \alpha_{\tau(j)} \\
\leqslant & \frac{1}{4}\left(1+\frac{1}{2 \log _{2}^{1 / 2}(a)}+\frac{\log _{2}(a)}{2^{3} \log _{2}(a)}+\cdots+\frac{1}{2^{v-1}}\right) \leqslant \frac{1}{2}, \\
& a=4 \tilde{M} K \sqrt{s} .
\end{aligned}
$$

Step IV. $B_{5} \Rightarrow(v)$. This step is identical to Step IV in the proof of Proposition 7.3.

Step $V$. The strong balancing property, (7.18) and (7.19) $\Rightarrow \mathbb{P}\left(A_{1}^{c} \cup A_{2}^{c} \cup\right.$ $\left.\widetilde{B}_{1}^{c} \cup \widetilde{B}_{2}^{c} \cup B_{3}^{c}\right) \leqslant 5 \gamma$. We start by bounding $\mathbb{P}\left(\widetilde{B}_{1}^{c}\right)$ and $\mathbb{P}\left(\widetilde{B}_{2}^{c}\right)$. Note that by Proposition 7.11(ii) it follows that $\mathbb{P}\left(\widetilde{B}_{1}^{c}\right) \leqslant \gamma$ and $\mathbb{P}\left(\widetilde{B}_{2}^{c}\right) \leqslant \gamma$ as long as the strong balancing property is satisfied and

$$
1 \gtrsim \Lambda \cdot \log \left(\frac{4}{\gamma}(\tilde{\theta}-s)\right), \quad 1 \gtrsim \Upsilon \cdot \log \left(\frac{4}{\gamma}(\tilde{\theta}-s)\right),
$$

where $\tilde{\theta}=\tilde{\theta}\left(\left\{q_{k}^{i}\right\}_{k=1}^{r}, 1 / 8,\left\{N_{k}\right\}_{k=1}^{r}, s, M\right)$ for $i=1,2$ and where $\tilde{\theta}$ is defined in Proposition 7.11(ii) and $\Lambda$ and $\Upsilon$ are defined in (7.73) and (7.74). Note that it is easy to see that we have

$$
\begin{aligned}
& \mid\{j \in \mathbb{N}: \max _{\substack{\Gamma_{1} \subset\{1, \ldots, M\}, \Gamma_{2, j} \subset\left\{N_{j-1}+1, \ldots, N_{j}\right\},}} \underbrace{}_{\substack{\mid=s \\
j=1, \ldots, r}} \\
& \left.\left\|P_{\Gamma_{1}} U^{*}\left(\left(q_{1}^{i}\right)^{-1} P_{\Gamma_{2,1}} \oplus \cdots \oplus\left(q_{r}^{i}\right)^{-1} P_{\Gamma_{2, r}}\right) U e_{j}\right\|>\frac{1}{8 \sqrt{s}}\right\} \mid \leqslant \tilde{M},
\end{aligned}
$$

where

$$
\tilde{M}=\min \left\{i \in \mathbb{N}: \max _{j \geqslant i}\left\|P_{N} U P_{\{j\}}\right\| \leqslant 1 /(K 32 \sqrt{s})\right\},
$$

and this follows from the choice in (7.63) where $q_{k}^{1}=q_{k}^{2}=\frac{1}{4} q_{k}$ for $1 \leqslant k \leqslant r$. Thus, it immediately follows that (7.18) and (7.19) imply (7.95). To bound $\mathbb{P}\left(B_{3}^{c}\right)$, we first deduce as in Step V of the proof of Proposition 7.3 that

$$
\mathbb{P}\left(\left\|P_{\Delta} U^{*}\left(\frac{1}{q_{1}} P_{\Omega_{1}} \oplus \cdots \oplus \frac{1}{q_{r}} P_{\Omega_{r}}\right) U P_{\Delta}-P_{\Delta}\right\|>1 / 4,\right) \leqslant \gamma / 2
$$


when the strong balancing property and (7.18) holds. For the second part of $B_{3}$, we know from the choice of $\tilde{M}$ that

$$
\max _{i \geqslant \tilde{M}}\left\|\left(q_{1}^{-1 / 2} P_{\Omega_{1}} \oplus \cdots \oplus q_{r}^{-1 / 2} P_{\Omega_{r}}\right) U e_{i}\right\| \leqslant \sqrt{\frac{5}{4}}
$$

and we may deduce from Proposition 7.13 that

$$
\mathbb{P}\left(\max _{i \in \Delta^{c} \cap\{1, \ldots, \tilde{M}\}}\left\|\left(q_{1}^{-1 / 2} P_{\Omega_{1}} \oplus \cdots \oplus q_{r}^{-1 / 2} P_{\Omega_{r}}\right) U e_{i}\right\|>\sqrt{5 / 4}\right) \leqslant \frac{\gamma}{2},
$$

whenever

$1 \gtrsim \log \left(\frac{2 \tilde{M}}{\gamma}\right) \cdot \max _{1 \leqslant k \leqslant r}\left\{\left(\frac{N_{k}-N_{k-1}}{m_{k}}-1\right) \mu_{\mathbf{N}, \mathbf{M}}(k, l)\right\}, \quad l=1, \ldots, r-1, \infty$,

which is true whenever (7.18) holds, since by a similar argument to (7.78),

$$
\kappa_{\mathbf{N}, \mathbf{M}}(k, \infty)+\sum_{j=1}^{r-1} \kappa_{\mathbf{N}, \mathbf{M}}(k, j) \geqslant \mu_{\mathbf{N}, \mathbf{M}}(k, l), \quad l=1, \ldots, r-1, \infty .
$$

Thus, $\mathbb{P}\left(B_{3}^{c}\right) \leqslant \gamma$. As for bounding $\mathbb{P}\left(A_{1}^{c}\right)$ and $\mathbb{P}\left(A_{2}^{c}\right)$, observe that by the strong balancing property $\tilde{M} \geqslant M$, thus this is done exactly as in Step $\mathrm{V}$ of the proof of Proposition 7.3.

Step VI. The strong balancing property, (7.18) and (7.19) $\Rightarrow \mathbb{P}\left(B_{4}^{c}\right) \leqslant \gamma$. To see this, define the random variables $X_{1}, \ldots, X_{u-2}$ as in (7.79). Let $\pi$ be defined as in Step VI of the proof of Proposition 7.3. Then it suffices to show that (7.18) and (7.19) imply that for $l=1, \ldots,\left(\begin{array}{c}u-2 \\ u-v\end{array}\right)$ and $j=0, \ldots, u-v-2$, we have

$$
\begin{aligned}
& \frac{1}{4} \geqslant \mathbb{P}\left(X_{\pi(l)_{u-v-j}}=1 \mid X_{\pi(l)_{1}}=1, \ldots, X_{\pi(l)_{u-v-(j+1)}}=1\right), \\
& \frac{1}{4} \geqslant \mathbb{P}\left(X_{\pi(l)_{1}}=1\right) .
\end{aligned}
$$

Claim. The strong balancing property, (7.18) and (7.19) $\Rightarrow$ (7.96). To prove the claim we first observe that $X_{j}=0$ when

$$
\begin{aligned}
& \left\|\left(P_{\Delta}-P_{\Delta} U^{*}\left(\frac{1}{q_{1}^{i}} P_{\Omega_{1}^{i}} \oplus \cdots \oplus \frac{1}{q_{r}^{i}} P_{\Omega_{r}^{i}}\right) U P_{\Delta}\right) Z_{i-1}\right\|_{l^{\infty}} \leqslant \frac{1}{2}\left\|Z_{i-1}\right\|_{l^{\infty}} \\
& \left\|P_{\Delta}^{\perp} U^{*}\left(\frac{1}{q_{1}^{i}} P_{\Omega_{1}^{i}} \oplus \cdots \oplus \frac{1}{q_{r}^{i}} P_{\Omega_{r}^{i}}\right) U P_{\Delta} Z_{i-1}\right\|_{l^{\infty}} \leqslant \frac{1}{4} \log _{2}(4 K \tilde{M} \sqrt{s})\left\|Z_{i-1}\right\|_{l^{\infty}}, \\
& \quad i=j+2 .
\end{aligned}
$$


Thus, by again recalling from (7.63) that $q_{k}^{3}=q_{k}^{4}=\cdots=q_{k}^{u}=\tilde{q}_{k}, 1 \leqslant k \leqslant r$, and by choosing $\tilde{\gamma}=1 / 4$ in (7.48) in Proposition 7.12 and $\tilde{\gamma}=1 / 4$ in (ii) in Proposition 7.11, we conclude that (7.96) follows when the strong balancing property is satisfied as well as (7.86) and (7.87). and

$$
\begin{aligned}
& \frac{\log _{2}(4 K \tilde{M} \sqrt{s})}{\log (16(\tilde{M}-s))} \geqslant C_{2} \cdot \tilde{q}_{k}^{-1} \cdot\left(\sum_{l=1}^{r-1} \kappa_{\mathbf{N}, \mathbf{M}}(k, l)+\kappa_{\mathbf{N}, \mathbf{M}}(k, \infty)\right), \\
& \frac{\log _{2}(4 K \tilde{M} \sqrt{s})}{\log (16(\tilde{M}-s))} \geqslant C_{2} \cdot\left(\sum_{l=1}^{r}\left(\tilde{q}_{k}^{-1}-1\right) \cdot \mu_{\mathbf{N}, \mathbf{M}}(k, l) \cdot \tilde{s}_{k}\right),
\end{aligned}
$$

where $k=1, \ldots, r$ and $l=1, \ldots, r-1, \infty$ and for $K=\max _{1 \leqslant k \leqslant r}\left(N_{k}-\right.$ $\left.N_{k-1}\right) / m_{k}$. for some constants $C_{1}$ and $C_{2}$. Thus, to prove the claim we must demonstrate that (7.18) and (7.19) $\Rightarrow$ (7.86), (7.87), (7.97) and (7.98). This is done by repeating Stage 1 and Stage 2 in Step VI of the proof of Proposition 7.3 almost verbatim, except replacing $M$ by $\tilde{M}$.

7.4. Proofs of Theorem 6.2 and Proposition 6.4. Throughout this section, we use the notation

$$
\hat{f}(\xi)=\int_{\mathbb{R}} f(x) e^{-i x \xi} d x,
$$

to denote the Fourier transform of a function $f \in L^{1}(\mathbb{R})$.

7.4.1. Setup. We first introduce the wavelet sparsity and Fourier sampling bases that we consider, and in particular, their orderings. Consider an orthonormal basis of compactly supported wavelets with an MRA [27, 28]. For simplicity, suppose that $\operatorname{supp}(\Psi)=\operatorname{supp}(\Phi)=[0, a]$ for some $a \geqslant 1$, where $\Psi$ and $\Phi$ are the mother wavelet and scaling function respectively. For later use, we recall the following three properties of any such wavelet basis:

(1) There exist $\alpha \geqslant 1, C_{\Psi}$ and $C_{\Phi}>0$, such that

$$
|\hat{\Phi}(\xi)| \leqslant \frac{C_{\Phi}}{(1+|\xi|)^{\alpha}}, \quad|\hat{\Psi}(\xi)| \leqslant \frac{C_{\Psi}}{(1+|\xi|)^{\alpha}} .
$$

See [28, Equation (7.1.4)]. We denote $\max \left\{C_{\Psi}, C_{\Phi}\right\}$ by $C_{\Phi, \Psi}$.

(2) $\Psi$ has $v \geqslant 1$ vanishing moments and $\hat{\Psi}(z)=(-i z)^{v} \theta_{\Psi}(z)$ for some bounded function $\theta_{\Psi}$ (see [64, pages 208 and 284]).

(3) $\|\hat{\Phi}\|_{L^{\infty}},\|\hat{\Psi}\|_{L^{\infty}} \leqslant 1$. 
REMARK 7.15. The three properties above are based on the standard setup for an MRA, however, we also consider a stronger assumption on the decay of the Fourier transform of derivatives of the scaling function and the mother wavelet. In particular, in addition, we sometimes assume that for $C>0$ and $\alpha \geqslant 1.5$,

$$
\left|\hat{\Phi}^{(k)}(\xi)\right| \leqslant \frac{C}{(1+|\xi|)^{\alpha}}, \quad\left|\hat{\Psi}^{(k)}(\xi)\right| \leqslant \frac{C}{(1+|\xi|)^{\alpha}}, \quad \xi \in \mathbb{R}, k=0,1,2,
$$

where $\hat{\Phi}^{(k)}$ and $\hat{\Psi}^{(k)}$ denotes the $k$ th derivative of the Fourier transform of $\Phi$ and $\Psi$ respectively. As is evident from Theorem 6.2, the faster decay, the closer the relationship between $N$ and $M$ in the balancing property gets to linear. Also, faster decay and more vanishing moments yield a closer to block-diagonal structure of the matrix $U$.

We now wish to construct a wavelet basis for the compact interval $[0, a]$. The most standard approach is to consider the following collection of functions

$$
\begin{gathered}
\Lambda_{a}=\left\{\Phi_{k}, \Psi_{j, k}: \operatorname{supp}\left(\Phi_{k}\right)^{o} \cap[0, a] \neq \emptyset, \operatorname{supp}\left(\Psi_{j, k}\right)^{o} \cap[0, a] \neq \emptyset,\right. \\
\left.j \in \mathbb{Z}_{+}, k \in \mathbb{Z}\right\}
\end{gathered}
$$

where $\Phi_{k}=\Phi(\cdot-k)$, and $\Psi_{j, k}=2^{j / 2} \Psi\left(2^{j} \cdot-k\right)$. (the notation $K^{o}$ denotes the interior of a set $K \subseteq \mathbb{R}$ ). This gives

$$
\begin{aligned}
\left\{f \in \mathrm{L}^{2}(\mathbb{R}): \operatorname{supp}(f) \subseteq[0, a]\right\} & \subseteq \overline{\operatorname{span}\left\{\varphi: \varphi \in \Lambda_{a}\right\}} \\
& \subseteq\left\{f \in \mathrm{L}^{2}(\mathbb{R}): \operatorname{supp}(f) \subseteq\left[-T_{1}, T_{2}\right]\right\},
\end{aligned}
$$

where $T_{1}, T_{2}>0$ are such that $\left[-T_{1}, T_{2}\right]$ contains the support of all functions in $\Lambda_{a}$. Note that the inclusions may be proper (but not always, as is the case with the Haar wavelet). It is easy to see that

$$
\begin{aligned}
\Psi_{j, k} \notin \Lambda_{a} & \Longleftrightarrow \frac{a+k}{2^{j}} \leqslant 0, \quad a \leqslant \frac{k}{2^{j}}, \\
\Phi_{k} \notin \Lambda_{a} & \Longleftrightarrow a+k \leqslant 0, \quad a \leqslant k,
\end{aligned}
$$

and therefore

$$
\Lambda_{a}=\left\{\Phi_{k}:|k|=0, \ldots,\lceil a\rceil-1\right\} \cup\left\{\Psi_{j, k}: j \in \mathbb{Z}_{+}, k \in \mathbb{Z},-\lceil a\rceil<k<2^{j}\lceil a\rceil\right\} .
$$

We order $\Lambda_{a}$ in increasing order of wavelet resolution as follows:

$$
\begin{aligned}
& \left\{\Phi_{-\lceil a\rceil+1}, \ldots, \Phi_{-1}, \Phi_{0}, \Phi_{1}, \ldots, \Phi_{\lceil a\rceil-1},\right. \\
& \left.\Psi_{0,-\lceil a\rceil+1}, \ldots, \Psi_{0,-1}, \Psi_{0,0}, \Psi_{0,1}, \ldots, \Psi_{0,\lceil a\rceil-1}, \Psi_{1,-\lceil a\rceil+1}, \ldots\right\},
\end{aligned}
$$


and then we finally denote the functions according to this ordering by $\left\{\varphi_{j}\right\}_{j \in \mathbb{N}}$. By the definition of $\Lambda_{a}$, we let $T_{1}=\lceil a\rceil-1$ and $T_{2}=2\lceil a\rceil-1$. Finally, for $R \in \mathbb{N}$, let $\Lambda_{R, a}$ contain all wavelets in $\Lambda_{a}$ with resolution less than $R$, so that

$$
\Lambda_{R, a}=\left\{\varphi \in \Lambda_{a}: \varphi=\Psi_{j, k}, 0 \leqslant j<R, \text { or } \varphi=\Phi_{k}\right\} .
$$

We also denote the size of $\Lambda_{R, a}$ by $W_{R}$. It is easy to verify that

$$
W_{R}=2^{R}\lceil a\rceil+(R+1)(\lceil a\rceil-1) .
$$

Having constructed an orthonormal wavelet system for $[0, a]$, we now introduce the appropriate Fourier sampling basis. We must sample at a rate that is at least that of the Nyquist rate. Hence we let $\omega \leqslant 1 /\left(T_{1}+T_{2}\right)$ be the sampling density (note that $1 /\left(T_{1}+T_{2}\right)$ is the Nyquist criterion for functions supported on $\left.\left[-T_{1}, T_{2}\right]\right)$. For simplicity, we assume throughout that

$$
\omega \in\left(0,1 /\left(T_{1}+T_{2}\right)\right), \quad \omega^{-1} \in \mathbb{N},
$$

and remark that this assumption is an artefact of our proofs and is not necessary in practice. The Fourier sampling vectors are now defined as follows.

$$
\psi_{j}(x)=\sqrt{\omega} e^{-2 \pi i j \omega x} \chi_{\left[-T_{1} /\left(\omega\left(T_{1}+T_{2}\right)\right), T_{2} /\left(\omega\left(T_{1}+T_{2}\right)\right)\right]}(x), \quad j \in \mathbb{Z} .
$$

This gives an orthonormal sampling basis for the space $\left\{f \in \mathrm{L}^{2}(\mathbb{R}): \operatorname{supp}(f) \subseteq\right.$ $\left.\left[-T_{1}, T_{2}\right]\right\}$. Since $\Lambda_{a}$ is an orthonormal system for this space, it follows that the infinite matrix

$$
U=\left(\begin{array}{cccc}
u_{11} & u_{12} & u_{13} & \cdots \\
u_{21} & u_{22} & u_{23} & \cdots \\
u_{31} & u_{32} & u_{33} & \cdots \\
\vdots & \vdots & \vdots & \ddots
\end{array}\right), \quad u_{i j}=\left\langle\varphi_{j}, \tilde{\psi}_{i}\right\rangle
$$

is an isometry, where $\left\{\varphi_{j}\right\}_{j \in \mathbb{N}}$ represents the wavelets ordered according to (7.102) and $\left\{\tilde{\psi}_{j}\right\}_{j \in \mathbb{N}}$ is the standard ordering of the Fourier basis (7.106) over $\mathbb{N}\left(\tilde{\psi}_{1}=\psi_{0}\right.$, $\tilde{\psi}_{2 n}=\psi_{n}$ and $\tilde{\psi}_{2 n+1}=\psi_{-n}$ ). With slight abuse of notation it is this ordering that we are using in Theorem 6.2.

7.4.2. Some preliminary estimates. Throughout this section, we assume the setup and notation introduced above.

THEOREM 7.16. Let $U$ be the matrix of the Fourier/wavelets pair introduced in (7.107) with sampling density $\omega$ as in (7.105). Suppose that $\Phi$ and $\Psi$ satisfy the decay estimate (7.100) with $\alpha \geqslant 1$ and that $\Psi$ has $v \geqslant 1$ vanishing moments. Then the following holds. 
(i) We have $\mu(U) \geqslant \omega$.

(ii) We have that

$$
\begin{aligned}
& \mu\left(P_{N}^{\perp} U\right) \leqslant \frac{C_{\Phi, \Psi}^{2}}{\pi N(2 \alpha-1)(1+1 /(2 \alpha-1))^{2 \alpha}}, \quad N \in \mathbb{N}, \\
& \mu\left(U P_{N}^{\perp}\right) \leqslant\|\Psi\|_{L^{\infty}}^{2} \frac{4 \omega\lceil a\rceil}{N}, \quad N \geqslant 2\lceil a\rceil+2(\lceil a\rceil-1),
\end{aligned}
$$

and consequently $\mu\left(P_{N}^{\perp} U\right), \mu\left(U P_{N}^{\perp}\right)=\mathcal{O}\left(N^{-1}\right)$.

(iii) If the wavelet and scaling function satisfy the decay estimate (7.100) with $\alpha>1 / 2$, then, for $R$ and $N$ such that $\omega^{-1} 2^{R} \leqslant N$ and $M=\left|\Lambda_{R, a}\right|$ (recall the definition of $\Lambda_{R, a}$ from (7.103)),

$$
\mu\left(P_{N}^{\perp} U P_{M}\right) \leqslant \frac{C_{\Phi, \Psi}^{2}}{\pi^{2 \alpha} \omega^{2 \alpha-1}}\left(2^{R-1} N^{-1}\right)^{2 \alpha-1} N^{-1} .
$$

(iv) If the wavelet has $v \geqslant 1$ vanishing moments, $\omega^{-1} 2^{R} \geqslant N$ and $M=\left|\Lambda_{R, a}\right|$ with $R \geqslant 1$, then

$$
\mu\left(P_{N} U P_{M}^{\perp}\right) \leqslant \frac{\omega}{2^{R}} \cdot\left(\frac{\pi \omega N}{2^{R}}\right)^{2 v} \cdot\left\|\theta_{\Psi}\right\|_{L^{\infty}}^{2},
$$

where $\theta_{\Psi}$ is the function such that $\hat{\Psi}(z)=(-i z)^{v} \theta_{\Psi}(z)$ (see above).

Proof. Note that $\mu(U) \geqslant\left|\left\langle\Phi, \psi_{0}\right\rangle\right|^{2}=\omega|\hat{\Phi}(0)|^{2}$, moreover, it is known that $\hat{\Phi}(0)=1$ [51, Ch. 2, Theorem 1.7]. Thus, (i) follows.

To show (ii), let $R \in \mathbb{N},-\lceil a\rceil<j<2^{R}\lceil a\rceil$ and $k \in \mathbb{Z}$. Then, by the choice of $j$, we have that $\Psi_{R, j}$ is supported on $\left[-T_{1}, T_{2}\right]$. Also, $\psi_{k}(x)=$ $\sqrt{\omega} e^{-2 \pi i k \omega x} \chi_{\left[-T_{1} /\left(\omega\left(T_{1}+T_{2}\right)\right), T_{2} /\left(\omega\left(T_{1}+T_{2}\right)\right)\right]}(x)$. Thus, since by $(7.105)$ we have $\omega \in\left(0,1 /\left(T_{1}+T_{2}\right)\right)$, it follows that

$$
\begin{aligned}
\left\langle\Psi_{R, j}, \psi_{k}\right\rangle & =\sqrt{\omega} \int_{-T_{1} /\left(\omega\left(T_{1}+T_{2}\right)\right)}^{T_{2} /\left(\omega\left(T_{1}+T_{2}\right)\right)} \Psi_{R, j}(x) e^{2 \pi i \omega k x} d x \\
& =\sqrt{\omega} \hat{\Psi}_{R, j}(-2 \pi \omega k)=\sqrt{\frac{\omega}{2^{R}}} \hat{\Psi}\left(\frac{-2 \pi k \omega}{2^{R}}\right) e^{2 \pi i \omega k j / 2^{R}} .
\end{aligned}
$$

Also, similarly, it follows that

$$
\begin{aligned}
\left\langle\Phi_{j}, \psi_{k}\right\rangle=\sqrt{\omega} \int_{-T_{1} /\left(\omega\left(T_{1}+T_{2}\right)\right)}^{T_{2} /\left(\omega\left(T_{1}+T_{2}\right)\right)} \Phi_{j}(x) e^{2 \pi i \omega k x} d x & =\sqrt{\omega} \hat{\Phi}_{j}(-2 \pi k \omega) \\
& =\sqrt{\omega} \hat{\Phi}(-2 \pi k \omega) e^{2 \pi i \omega k j}
\end{aligned}
$$


Thus, the decay estimate in (7.100) yields

$$
\begin{aligned}
\mu\left(P_{N}^{\perp} U\right) & \leqslant \sup _{|k| \geqslant N / 2} \max _{\varphi \in \Lambda_{a}}\left|\left\langle\varphi, \psi_{k}\right\rangle\right|^{2} \\
& =\max \left\{\sup _{|k| \geqslant N / 2} \max _{R \in \mathbb{Z}_{+}} \frac{\omega}{2^{R}}\left|\hat{\Psi}\left(\frac{-2 \pi \omega k}{2^{R}}\right)\right|^{2}, \omega \sup _{|k| \geqslant N / 2}|\hat{\Phi}(-2 \pi \omega k)|^{2}\right\} \\
& \leqslant \max _{|k| \geqslant N / 2} \max _{R \in \mathbb{Z}_{+}} \frac{\omega}{2^{R}} \frac{C_{\Phi, \Psi}^{2}}{\left(1+\left|2 \pi \omega k 2^{-R}\right|\right)^{2 \alpha}} \leqslant \max _{R \in \mathbb{Z}_{+}} \frac{\omega}{2^{R}} \frac{C_{\Phi, \Psi}^{2}}{\left(1+\left|\pi \omega N 2^{-R}\right|\right)^{2 \alpha}} .
\end{aligned}
$$

The function $f(x)=x^{-1}(1+\pi \omega N / x)^{-2 \alpha}$ on $[1, \infty)$ satisfies $f^{\prime}(\pi \omega N(2 \alpha-1))=$ 0 . Hence

$$
\mu\left(P_{N}^{\perp} U\right) \leqslant \frac{C_{\Phi, \Psi}^{2}}{\pi N(2 \alpha-1)(1+1 /(2 \alpha-1))^{2 \alpha}},
$$

which gives the first part of (ii). For the second part, we first recall the definition of $W_{R}$ for $R \in \mathbb{N}$ from (7.104). Then, given any $N \in \mathbb{N}$ such that $N \geqslant W_{1}=$ $2\lceil a\rceil+2(\lceil a\rceil-1)$, let $R$ be such that $W_{R} \leqslant N<W_{R+1}$. Then, for each $n \geqslant N$, there exists some $j \geqslant R$ and $l \in \mathbb{Z}$ such that the $n^{\text {th }}$ element via the ordering (7.102) is $\varphi_{n}=\Psi_{j, l}$ (note that we only need $\Psi_{j, l}$ here and not $\Phi_{j}$ as we have chosen $N \geqslant W_{1}$ ). Hence, by using (7.108),

$$
\begin{aligned}
\mu\left(U P_{N}^{\perp}\right) & =\max _{n \geqslant N} \max _{k \in \mathbb{Z}}\left|\left\langle\varphi_{n}, \psi_{k}\right\rangle\right|^{2}=\max _{j \geqslant R} \max _{k \in \mathbb{Z}} \frac{\omega}{2^{j}}\left|\hat{\Psi}\left(\frac{-2 \pi \omega k}{2^{j}}\right)\right|^{2} \\
& \leqslant\|\hat{\Psi}\|_{L^{\infty}}^{2} \frac{\omega}{2^{R}} \leqslant 4\|\hat{\Psi}\|_{L^{\infty}}^{2} \frac{\omega\lceil a\rceil}{N}
\end{aligned}
$$

where the last line follows because $N<W_{R+1}=2^{R+1}\lceil a\rceil+(R+2)(\lceil a\rceil-1)$ implies that

$$
2^{-R}<\frac{1}{N}\left(2\lceil a\rceil+(R+2)(\lceil a\rceil-1) 2^{-R}\right) \leqslant \frac{4\lceil a\rceil}{N} .
$$

This concludes the proof of (ii).

To show (iii), let $R$ and $N$ be such that $\omega^{-1} 2^{R} \leqslant N$ and $M=\left|\Lambda_{R, a}\right|$. Observe that (7.108) and (7.109) together with the decay estimate in (7.100) yield

$$
\begin{aligned}
\mu\left(P_{N}^{\perp} U P_{W_{R}}\right) & \leqslant \max _{|k| \geqslant N / 2} \max _{\varphi \in \Lambda_{R, a}}\left|\left\langle\varphi, \psi_{k}\right\rangle\right|^{2} \\
& =\max \left\{\max _{|k| \geqslant N / 2} \max _{j<R} \frac{\omega}{2^{j}}\left|\hat{\Psi}\left(\frac{-2 \pi \omega k}{2^{j}}\right)\right|^{2}, \max _{|k| \geqslant N / 2}|\hat{\Phi}(-2 \pi \omega k)|^{2}\right\}
\end{aligned}
$$




$$
\begin{aligned}
& \leqslant \max _{|k| \geqslant N / 2} \max _{j<R} \frac{\omega}{2^{j}} \frac{C_{\Phi, \Psi}^{2}}{\left(1+\left|2 \pi \omega k 2^{-j}\right|\right)^{2 \alpha}} \leqslant \max _{k \geqslant N / 2} \max _{j<R} \frac{C_{\Phi, \Psi}^{2}}{\pi^{2 \alpha} \omega^{2 \alpha-1}} \frac{2^{j(2 \alpha-1)}}{(2 k)^{2 \alpha}} \\
& =\frac{C_{\Phi, \Psi}^{2}}{\pi^{2 \alpha} \omega^{2 \alpha-1}}\left(2^{R-1} N^{-1}\right)^{2 \alpha-1} N^{-1},
\end{aligned}
$$

and this colludes the proof of (iii).

To show (iv), first note that because $R \geqslant 1$, for all $n>W_{R}, \varphi_{n}=\Psi_{j, k}$ for some $j \geqslant 0$ and $k \in \mathbb{Z}$. Then, recalling the properties of Daubechies wavelets with $v$ vanishing moments, and by using (7.108) we get that

$$
\begin{aligned}
\mu\left(P_{N} U P_{W_{R}}^{\perp}\right) & =\max _{n>W_{R}} \max _{|k| \leqslant N / 2}\left|\left\langle\varphi_{n}, \psi_{k}\right\rangle\right|^{2}=\max _{j \geqslant R} \max _{|k| \leqslant N / 2} \frac{\omega}{2^{j}}\left|\hat{\Psi}\left(\frac{-2 \pi \omega k}{2^{j}}\right)\right|^{2} \\
& \leqslant \frac{\omega}{2^{R}} \cdot\left(\frac{\pi \omega N}{2^{R}}\right)^{2 v} \cdot\left\|\theta_{\Psi}\right\|_{L^{\infty}}^{2},
\end{aligned}
$$

as required.

COROllary 7.17. Let $\mathbf{N}$ and $\mathbf{M}$ be as in Theorem 6.2 and recall the definition of $\mu_{\mathbf{N}, \mathbf{M}}(k, j)$ in (4.3). Suppose that $\Phi$ and $\Psi$ satisfy the decay estimate (7.100) with $\alpha \geqslant 1$ and that $\Psi$ has $v \geqslant 1$ vanishing moments. Then,

for $k \geqslant 2, \quad \mu_{\mathbf{N}, \mathbf{M}}(k, j) \leqslant B_{\Phi, \Psi} \cdot \begin{cases}\frac{\sqrt{\omega}}{\sqrt{N_{k-1} 2^{R_{j-1}}}} \cdot\left(\frac{\omega N_{k}}{2^{R_{j-1}}}\right)^{v} & j \geqslant k+1, \\ \frac{1}{N_{k-1}}\left(\frac{2^{R_{j-1}}}{\omega N_{k-1}}\right)^{\alpha-1 / 2} & j \leqslant k-1,(7.110) \\ \frac{1}{N_{k-1}} & j=k,\end{cases}$

for $k \geqslant 2, \quad \mu_{\mathbf{N}, \mathbf{M}}(k, \infty) \leqslant B_{\Phi, \Psi} \cdot \begin{cases}\frac{\sqrt{\omega}}{\sqrt{N_{k-1} 2^{R_{r-1}}} \cdot\left(\frac{\omega N_{k}}{2^{R_{r-1}}}\right)^{v}} \quad k \leqslant r-1, \\ \frac{1}{N_{r-1}} & k=r,\end{cases}$

$$
\mu_{\mathbf{N}, \mathbf{M}}(1, j) \leqslant B_{\Phi, \Psi} \cdot \begin{cases}\frac{\sqrt{\omega}}{\sqrt{2^{R_{j-1}}}} \cdot\left(\frac{\omega N_{1}}{2^{R_{j-1}}}\right)^{v} & j \geqslant 2, \\ 1 & j=1,\end{cases}
$$

$$
\mu_{\mathbf{N}, \mathbf{M}}(1, \infty) \leqslant B_{\Phi, \Psi} \cdot \frac{\sqrt{\omega}}{\sqrt{2^{R_{r-1}}}} \cdot\left(\frac{\omega N_{1}}{2^{R_{r-1}}}\right)^{v},
$$

where $B_{\Phi, \Psi}$ is a constant which depends only on $\Phi$ and $\Psi$ and $R_{0}=0$. 
Proof. Throughout this proof, $B_{\Phi, \Psi}$ is a constant which depends only on $\Phi$ and $\Psi$, although its value may change from instance to instance. Note that

$$
\begin{aligned}
\mu_{\mathbf{N}, \mathbf{M}}(k, j) & =\sqrt{\mu\left(P_{N_{k}}^{N_{k-1}} U P_{M_{j}}^{M_{j-1}}\right) \cdot \mu\left(P_{N_{k}}^{N_{k-1}} U\right)} \\
& \leqslant B_{\Phi, \Psi} N_{k-1}^{-1 / 2} \sqrt{\mu\left(P_{N_{k}}^{N_{k-1}} U P_{M_{j}}^{M_{j-1}}\right)}, \quad k \geqslant 2, j \in\{1, \ldots, r\},
\end{aligned}
$$

since we have $\mu\left(P_{N_{k-1}}^{\perp} U\right) \leqslant B_{\Phi, \Psi} N_{k-1}^{-1}$ by (ii) of Theorem 7.16. Also, clearly

$$
\mu_{\mathbf{N}, \mathbf{M}}(1, j)=\sqrt{\mu\left(P_{N_{1}}^{N_{0}} U P_{M_{j}}^{M_{j-1}}\right) \cdot \mu\left(P_{N_{1}}^{N_{0}} U\right)} \leqslant B_{\Phi, \Psi} \sqrt{\mu\left(P_{N_{1}}^{N_{0}} U P_{M_{j}}^{M_{j-1}}\right)},
$$

for $j \in\{1, \ldots, r\}$. Thus, for $k \geqslant 2$, it follows that $\mu_{\mathbf{N}, \mathbf{M}}(k, k) \leqslant \mu\left(P_{N_{k-1}}^{\perp} U\right) \leqslant$ $B_{\Phi, \Psi}\left(1 / N_{k-1}\right)$, yielding the last part of (7.110). Also, the last part of (7.112) is clear from (7.115).

As for the middle part of (7.110), note that for $k \geqslant 2$, and with $j \leqslant k-1$, we may use (iii) of Theorem 7.16 to obtain

$$
\sqrt{\mu\left(P_{N_{k}}^{N_{k-1}} U P_{M_{j}}^{M_{j-1}}\right)} \leqslant \sqrt{\mu\left(P_{N_{k-1}}^{\perp} U P_{M_{j}}\right)} \leqslant B_{\Phi, \Psi} \cdot \frac{1}{\sqrt{N_{k-1}}}\left(\frac{2^{R_{j-1}}}{\omega N_{k-1}}\right)^{\alpha-1 / 2},
$$

and thus, in combination with (7.114), we obtain the $j \leqslant k-1$ part of (7.110). Observe that if $k \in\{1, \ldots, r\}$ and $j \geqslant k+1$, then by applying (iv) of Theorem 7.16, we obtain

$$
\sqrt{\mu\left(P_{N_{k}}^{N_{k-1}} U P_{M_{j}}^{M_{j-1}}\right)} \leqslant \sqrt{\mu\left(P_{N_{k}} U P_{M_{j-1}}^{\perp}\right)} \leqslant B_{\Phi, \Psi} \cdot \frac{\sqrt{\omega}}{\sqrt{2^{R_{j-1}}}} \cdot\left(\frac{\omega N_{k}}{2^{R_{j-1}}}\right)^{v} .
$$

Thus, by combining (7.116) with (7.114), we obtain the $j \geqslant k+1$ part of (7.110). Also, by combining (7.116) with (7.114) we get the $j \geqslant 2$ part of (7.112). Finally, recall that

$$
\mu_{\mathbf{N}, \mathbf{M}}(k, \infty)=\sqrt{\mu\left(P_{N_{k}}^{N_{k-1}} U P_{M_{r-1}}^{\perp}\right) \cdot \mu\left(P_{N_{k-1}}^{\perp} U\right)}
$$

and similarly to the above, (7.111) and (7.113) are direct consequences of parts (ii) and (iv) of Theorem 7.16.

The following lemmas inform us of the range of Fourier samples required for accurate reconstruction of wavelet coefficients. Specifically, Lemma 7.18 will provide a quantitative understanding of the balancing property, whilst Lemma 7.19 and Lemma 7.20 will be used in bounding the relative sparsity terms. 
Lemma 7.18 [67, Corollary 5.4]. Consider the setup in Section 7.4.1. Let the sampling density $\omega$ be such that $\omega^{-1} \in \mathbb{N}$ and suppose that there exists $C_{\Phi}, C_{\Psi}>0$ and $\alpha \geqslant 1.5$ such that

$$
\left|\hat{\Phi}^{(k)}(\xi)\right| \leqslant \frac{C_{\Phi}}{(1+|\xi|)^{\alpha}}, \quad\left|\hat{\Psi}^{(k)}(\xi)\right| \leqslant \frac{C_{\Psi}}{(1+|\xi|)^{\alpha}}, \quad \xi \in \mathbb{R}, \quad k=0,1,2 .
$$

Then given $\gamma \in(0,1)$, we have that $\left\|P_{M} U^{*} P_{N} U P_{M}-P_{M}\right\|_{1^{\infty} \rightarrow l^{\infty}} \leqslant \gamma$ wherever $N \geqslant C \gamma^{-1 /(2 \alpha-1)} M$ and $\left\|P_{M}^{\perp} U^{*} P_{N} U P_{M}\right\|_{l^{\infty} \rightarrow l^{\infty}} \leqslant \gamma$ wherever $N \geqslant C \gamma^{-1 /(\alpha-1)} M$ where $C$ is some constant independent of $N$ but dependent on $C_{\Phi}, C_{\Psi}$ and $\omega$.

LEMMA 7.19 [67, Lemma 5.1]. Let $\varphi_{k}$ denote the $k$ th wavelet via the ordering in (7.102). Let $R \in \mathbb{N}$ and $M \leqslant W_{R}$ be such that $\left\{\varphi_{j}: j \leqslant M\right\} \subset \Lambda_{R, a}$, where $W_{R}$ and $\Lambda_{R, a}$ are defined in (7.104) and (7.103), respectively. Also, let the sampling density $\omega$ be such that $\omega^{-1} \in \mathbb{N}$. Then for any $\gamma \in(0,1)$, we have that $\left\|P_{N}^{\perp} U P_{M}\right\| \leqslant \gamma$, whenever $N$ is such that

$$
N \geqslant \omega^{-1}\left(\frac{4 C_{\Phi}^{2}}{(2 \pi)^{2 \alpha} \cdot(2 \alpha-1)}\right)^{1 / 2 \alpha-1} \cdot 2^{R+1} \cdot \gamma^{-(2 / 2 \alpha-1)}
$$

and $C_{\Phi}$ is a constant depending on $\Phi$.

LEMMA 7.20. Let $\varphi_{k}$ denote the $k^{\text {th }}$ wavelet the ordering in (7.102). Let $R_{1}, R_{2} \in$ $\mathbb{N}$ with $R_{2}>R_{1}$, and $M_{1}, M_{2} \in \mathbb{N}$ with $M_{2}>M_{1}$ be such that

$$
\left\{\varphi_{j}: M_{2} \geqslant j>M_{1}\right\} \subset \Lambda_{R_{2}, a} \backslash \Lambda_{R_{1}, a},
$$

where $\Lambda_{R_{i}, a}$ is defined in (7.103). Then for any $\gamma \in(0,1)$

$$
\left\|P_{N} U P_{M_{2}}^{M_{1}}\right\| \leqslant \frac{\pi^{2}}{4}\left\|\theta_{\Psi}\right\|_{L^{\infty}} \cdot(2 \pi \gamma)^{v} \cdot \sqrt{\frac{1-2^{2 v\left(R_{1}-R_{2}\right)}}{1-2^{-2 v}}}
$$

whenever $N$ is such that $N \leqslant \gamma \omega^{-1} 2^{R_{1}}$.

Proof. Let $\eta \in l^{2}(\mathbb{N})$ be such that $\|\eta\|=1$. Note that, by the definition of $U$ in (7.107), it follows that

$$
\left\|P_{N} U P_{M_{2}}^{M_{1}} \eta\right\|^{2} \leqslant \sum_{|k| \leqslant N / 2}\left|\left\langle\psi_{k}, \sum_{j=M_{1}+1}^{M_{2}} \eta_{j} \varphi_{j}\right\rangle\right|^{2} \leqslant \sum_{|k| \leqslant N / 2}\left|\left\langle\psi_{k}, \sum_{l=R_{1}}^{R_{2}-1} \sum_{j \in \Delta_{l}} \eta_{\rho(l, j)} \Psi_{l, j}\right\rangle\right|^{2},
$$

where we have defined

$$
\Delta_{l}=\left\{j \in \mathbb{Z}: \Psi_{l, j} \in \Lambda_{l+1, a} \backslash \Lambda_{l, a}\right\}, \quad \rho:\left\{\left(l, \Delta_{l}\right)\right\}_{l \in \mathbb{N}} \rightarrow \mathbb{N} \backslash\left\{1, \ldots,\left|\Lambda_{1, a}\right|\right\}
$$


to be the bijection such that $\varphi_{\rho(l, j)}=\Psi_{l, j}$. Now, observe that we may argue as in the proof of Theorem 7.16 and use (7.108) to deduce that given $l \in \mathbb{N},-\lceil a\rceil<$ $j<2^{l}\lceil a\rceil$ and $k \in \mathbb{Z}$, we have that $\left\langle\Psi_{l, j}, \psi_{k}\right\rangle=\sqrt{\omega / 2^{l}} \hat{\Psi}\left(-\left(2 \pi \omega k / 2^{l}\right)\right) e^{2 \pi i \omega j k}$. Hence, it follows that

$$
\begin{aligned}
& \sum_{|k| \leqslant N / 2}\left|\left\langle\psi_{k}, \sum_{l=R_{1}}^{R_{2}-1} \sum_{j \in \Delta_{l}} \eta_{\rho(l, j)} \Psi_{l, j}\right\rangle\right|^{2} \\
& =\sum_{|k| \leqslant N / 2}\left|\sum_{l=R_{1}}^{R_{2}-1} \frac{\sqrt{\omega}}{\sqrt{2^{l}}} \sum_{j \in \Delta_{l}} \eta_{\rho(l, j)} \hat{\Psi}\left(-\frac{2 \pi \omega k}{2^{l}}\right) e^{2 \pi i \omega j k / 2^{l}}\right|^{2}
\end{aligned}
$$

which again gives us that

$$
\begin{aligned}
\left\|P_{N} U P_{M_{2}}^{M_{1}} \eta\right\|^{2} & \leqslant \sum_{|k| \leqslant N / 2}\left|\sum_{l=R_{1}}^{R_{2}-1} \frac{\sqrt{\omega}}{\sqrt{2^{l}}} \hat{\Psi}\left(-\frac{2 \pi \omega k}{2^{l}}\right) f^{[l]}\left(\frac{\omega k}{2^{l}}\right)\right|^{2} \\
& \leqslant \sum_{|k| \leqslant N / 2} \sum_{l=R_{1}}^{R_{2}-1}\left|\hat{\Psi}\left(-\frac{2 \pi \omega k}{2^{l}}\right)\right|^{2} \cdot \sum_{l=R_{1}}^{R_{2}-1}\left|\frac{\sqrt{\omega}}{\sqrt{2^{l}}} f^{[l]}\left(\frac{\omega k}{2^{l}}\right)\right|^{2} \\
& \leqslant \sum_{l=R_{1}}^{R_{2}-1} \max _{|k| \leqslant N / 2}\left|\hat{\Psi}\left(-\frac{2 \pi \omega k}{2^{l}}\right)\right|^{2} \cdot \sum_{l=R_{1}}^{R_{2}-1} \sum_{|k| \leqslant N / 2} \frac{\omega}{2^{l}}\left|f^{[l]}\left(\frac{\omega k}{2^{l}}\right)\right|^{2},
\end{aligned}
$$

where $f^{[l]}(z)=\sum_{j \in \Delta_{l}} \eta_{\rho(l, j)} e^{2 \pi i z j}$. Let $H=\chi_{[0,1)}$ and, for $l \in \mathbb{N},-\lceil a\rceil<j<$ $2^{j}\lceil a\rceil$, define $H_{l, j}=2^{l / 2} H\left(2^{l} \cdot-j\right)$. By the choice of $j$, we have that $H_{l, j}$ is supported on $\left[-T_{1}, T_{2}\right]$. Also, since by (7.105) we have $\omega \in\left(0,1 /\left(T_{1}+T_{2}\right)\right)$, we may argue as in (7.108) and find that $\left\langle H_{l, j}, \psi_{k}\right\rangle=\sqrt{\omega / 2^{l}} \hat{H}\left(-2 \pi k \omega / 2^{l}\right) e^{2 \pi i \omega k j / 2^{l}}$. Thus,

$$
\left\langle\sum_{j \in \Delta_{l}} \eta_{\rho(l, j)} H_{l, j}, \psi_{k}\right\rangle=\sqrt{\frac{\omega}{2^{l}}} \sum_{j \in \Delta_{l}} \eta_{\rho(l, j)} \hat{H}\left(\frac{-2 \pi k \omega}{2^{l}}\right) e^{2 \pi i \omega k j / 2^{l}} .
$$

It is straightforward to show that $\inf _{|x| \leqslant \pi}|\hat{H}(x)| \geqslant 2 / \pi$, and since $N \leqslant 2^{R_{1}} / \omega$, for each $l \geqslant R_{1}$, it follows directly from (7.118) and the definition of $f^{[l]}$ that

$$
\begin{aligned}
\sum_{|k| \leqslant N / 2} \frac{\omega}{2^{l}}\left|f^{[l]}\left(\frac{\omega k}{2^{l}}\right)\right|^{2} & \leqslant\left(\inf _{|x| \leqslant \pi}|\hat{H}(x)|^{2}\right)^{-1} \sum_{|k| \leqslant N / 2}\left|\left\langle\sum_{j \in \Delta_{l}} \eta_{\rho(l, j)} H_{l, j}, \psi_{k}\right\rangle\right|^{2} \\
& \leqslant \frac{\pi^{2}}{4}\left\|\sum_{j \in \Delta_{l}} \eta_{\rho(l, j)} H_{l, j}\right\|^{2} \leqslant \frac{\pi^{2}}{4}\left\|P_{\Delta_{l}} \eta\right\|^{2} .
\end{aligned}
$$


Hence, we immediately get that

$$
\sum_{l=R_{1}}^{R_{2}-1} \sum_{|k| \leqslant N / 2} \frac{\omega}{2^{l}}\left|f^{[l]}\left(\frac{\omega k}{2^{l}}\right)\right|^{2} \leqslant \frac{\pi^{2}}{4} \sum_{l=R_{1}}^{R_{2}-1}\left\|P_{\Delta_{l}} \eta\right\|^{2} \leqslant \frac{\pi^{2}}{4}\|\eta\|^{2} \leqslant \frac{\pi^{2}}{4} .
$$

Also, since $\Psi$ has $v$ vanishing moments, we have that $\hat{\Psi}(z)=(-i z)^{v} \theta_{\Psi}(z)$ for some bounded $L^{\infty}$ function $\theta_{\Psi}$. Thus, since $N \leqslant \gamma \cdot 2^{R_{1}} / \omega$, we have

$$
\begin{aligned}
\sum_{l=R_{1}}^{R_{2}-1} \max _{|k| \leqslant N / 2}\left|\hat{\Psi}\left(\frac{2 \pi \omega k}{2^{l}}\right)\right|^{2} & \leqslant \frac{\pi^{2}}{4}\left\|\theta_{\Psi}\right\|_{L^{\infty}}^{2} \sum_{l=R_{1}}^{R_{2}-1}\left(2 \pi \gamma 2^{R_{1}-l}\right)^{2 v} \\
& \leqslant \frac{\pi^{2}}{4}(2 \pi \gamma)^{2 v}\left\|\theta_{\Psi}\right\|_{L^{\infty}}^{2} \frac{1-2^{2 v\left(R_{1}-R_{2}\right)}}{1-2^{-2 v}} .
\end{aligned}
$$

Thus, by applying (7.117), (7.118) and (7.119), it follows that

$$
\left\|P_{N} U P_{M_{2}}^{M_{1}} \eta\right\|^{2} \leqslant \frac{\pi^{2}}{4}\left\|\theta_{\Psi}\right\|_{L^{\infty}}^{2} \cdot(2 \pi \gamma)^{2 v} \frac{1-2^{2 v\left(R_{1}-R_{2}\right)}}{1-2^{-2 v}},
$$

and we have proved the desired estimate.

\subsubsection{The proofs.}

Proof of Theorem 6.2. In this proof, we let $B_{\Phi, \Psi}$ be some constant which depends only on $\Phi$ and $\Psi$, although its value may change from instance to instance. The assertions of the theorem will follow if we can show that the conditions in Theorem 5.3 are satisfied. We begin with condition (i). First observe that since $U$ is an isometry we have that $\left\|P_{M} U^{*} P_{N} U P_{M}-P_{M}\right\|_{l^{\infty}}=\left\|P_{M} U^{*} P_{N}^{\perp} U P_{M}\right\|_{l^{\infty} \rightarrow l^{\infty}} \leqslant$ $\sqrt{M}\left\|P_{N}^{\perp} U P_{M}\right\|$ and $\left\|P_{M}^{\perp} U^{*} P_{N} U P_{M}\right\|_{l^{\infty} \rightarrow l^{\infty}}=\left\|P_{M}^{\perp} U^{*} P_{N}^{\perp} U P_{M}\right\|_{l^{\infty} \rightarrow l^{\infty}} \leqslant$ $\sqrt{M}\left\|P_{N}^{\perp} U P_{M}\right\|$. So $N, K$ satisfy the strong balancing property with respect to $U, M$ and $s$ if

$$
\left\|P_{N}^{\perp} U P_{M}\right\| \leqslant \frac{1}{8}\left(M \log _{2}(4 K M \sqrt{s})\right)^{-1 / 2} .
$$

In the case of $\alpha \geqslant 1$, by applying Lemma 7.19 with

$$
\gamma=\frac{1}{8}\left(M \log _{2}(4 K M \sqrt{s})\right)^{-1 / 2},
$$

it follows that $N, K$ satisfy the strong balancing property with respect to $U, M, s$ whenever

$$
N \geqslant C_{\omega, \Phi} \cdot 2^{R+1} \cdot\left(\frac{1}{8}\left(M \log _{2}(4 K M \sqrt{s})\right)^{-1 / 2}\right)^{-(2 /(2 \alpha-1))},
$$


where $R$ is the smallest integer such that $M \leqslant W_{R}$ (where $W_{R}$ is defined in (7.104)) and $C_{\omega, \Phi}$ is a constant which depends only on the Fourier decay of $\Phi$ and $\omega$. By the choice of $R$, we have that $M=\mathcal{O}\left(2^{R}\right)$ since $W_{R}=\mathcal{O}\left(2^{R}\right)$ by (7.104). Thus, the strong balancing property holds provided that

$$
N \gtrsim M^{1+1 /(2 \alpha-1)} \cdot\left(\log _{2}(4 M K \sqrt{s})\right)^{1 /(2 \alpha-1)}
$$

where the constant involved depends only on $\omega$ and the Fourier decay of $\Phi$. Furthermore, if (7.101) holds, then a direct application of Lemma 7.18 gives that $N, K$ satisfy the strong balancing property with respect to $U, M, s$ whenever $N \gtrsim M \cdot\left(\log _{2}(4 K M \sqrt{s})\right)^{1 /(4 \alpha-2)}$. So, condition (i) of Theorem 6.2 implies condition (i) of Theorem 5.3.

To show that (ii) in Theorem 5.3 is satisfied, we need to demonstrate that

$$
1 \gtrsim \frac{N_{k}-N_{k-1}}{m_{k}} \cdot \log \left(\epsilon^{-1}\right) \cdot\left(\sum_{l=1}^{r} \mu_{\mathbf{N}, \mathbf{M}}(k, l) \cdot s_{l}\right) \cdot \log (K \tilde{M} \sqrt{s}),
$$

(with $\mu_{\mathbf{N}, \mathbf{M}}(k, r)$ replaced by $\mu_{\mathbf{N}, \mathbf{M}}(k, \infty)$, and also recall that $N_{0}=0$ ) and

$$
\begin{gathered}
m_{k} \gtrsim \hat{m}_{k} \cdot \log \left(\epsilon^{-1}\right) \cdot \log (K \tilde{M} \sqrt{s}), \\
1 \gtrsim \sum_{k=1}^{r}\left(\frac{N_{k}-N_{k-1}}{\hat{m}_{k}}-1\right) \cdot \mu_{\mathbf{N}, \mathbf{M}}(k, l) \cdot \tilde{s}_{k}, \quad \forall l=1, \ldots, r,
\end{gathered}
$$

where

$$
\tilde{M}=\min \left\{i \in \mathbb{N}: \max _{k \geqslant i}\left\|P_{N} U e_{k}\right\| \leqslant 1 /(32 K \sqrt{s})\right\} .
$$

We first consider (7.120). By applying the bounds (7.110) and (7.111) on the local coherences derived in Corollary 7.17, we have that (7.120) is implied by

$$
\begin{aligned}
\frac{m_{k}}{\left(N_{k}-N_{k-1}\right)} \gtrsim & B_{\Phi, \Psi} \cdot\left(\sum_{j=1}^{k-1} \frac{s_{j}}{N_{k-1}}\left(\frac{2^{R_{j-1}}}{\omega N_{k-1}}\right)^{\alpha-1 / 2}+\frac{s_{k}}{N_{k-1}}\right. \\
& \left.+\sum_{j=k+1}^{r} \frac{s_{j} \cdot \sqrt{\omega}}{\sqrt{N_{k-1} 2^{R_{j-1}}}} \cdot\left(\frac{\omega N_{k}}{2^{R_{j-1}}}\right)^{v}\right) \cdot \log \left(\epsilon^{-1}\right) \cdot \log (K \tilde{M} \sqrt{s}),
\end{aligned}
$$

where $k=2, \ldots, r$, and

$$
\frac{m_{1}}{N_{1}} \gtrsim B_{\Phi, \Psi} \cdot\left(s_{1}+\sum_{j=2}^{r} \frac{s_{j} \cdot \sqrt{\omega}}{\sqrt{2^{R_{j-1}}}} \cdot\left(\frac{\omega N_{1}}{2^{R_{j-1}}}\right)^{v}\right) \cdot \log \left(\epsilon^{-1}\right) \cdot \log (K \tilde{M} \sqrt{s}) .
$$


To obtain a bound on the value of $\tilde{M}$ in (7.122), observe that by Lemma 7.20, $\left\|P_{N} U P_{\{j\}}\right\| \leqslant 1 /(32 K \sqrt{s})$ whenever $j=\left|\Lambda_{J, a}\right|=\mathcal{O}\left(2^{J}\right)$ such that $2^{J} \geqslant$ $(32 K \sqrt{s})^{1 / v} \cdot N \cdot \omega$. Thus, $\tilde{M} \leqslant\left\lceil(32 K \sqrt{s})^{1 / v} \cdot N \cdot \omega\right\rceil$, and by recalling that $N_{k}=2^{R_{k}} \omega^{-1}$, we have that (7.123) is implied by

$$
\begin{aligned}
\frac{m_{k} \cdot N_{k-1}}{N_{k}-N_{k-1}} \gtrsim & B_{\Phi, \Psi} \cdot \log \left(\epsilon^{-1}\right) \cdot \log \left((K \sqrt{s})^{1+1 / v} N\right) \\
& \times\left(\sum_{j=1}^{k-1} s_{j} \cdot\left(2^{\alpha-1 / 2}\right)^{-\left(R_{k-1}-R_{j-1}\right)}+s_{k}+s_{k+1} \cdot 2^{-\left(R_{k}-R_{k-1}\right) / 2}\right. \\
& \left.+\sum_{j=k+2}^{r} s_{j} \cdot 2^{-\left(R_{j-1}-R_{k-1}\right) / 2} \cdot 2^{-v\left(R_{j-1}-R_{k}\right)}\right), \quad k \geqslant 2
\end{aligned}
$$

and when $k=1,(7.124)$ is implied by

$$
\begin{aligned}
\frac{m_{1}}{N_{1}} \gtrsim & B_{\Phi, \Psi} \cdot \log \left(\epsilon^{-1}\right) \cdot \log \left((K \sqrt{s})^{1+1 / v} N\right) \\
& \times\left(s_{1}+s_{2} \cdot 2^{-R_{1} / 2}+\sum_{j=k+2}^{r} s_{j} \cdot 2^{-\left(R_{j-1}-R_{k-1}\right) / 2} \cdot 2^{-v\left(R_{j-1}-R_{k}\right)}\right) .
\end{aligned}
$$

However, the condition (6.1) obviously implies (7.125) and (7.124), hence we have established that condition (6.1) implies (7.120). As for condition (7.121), we first derive upper bounds for the $\tilde{s}_{k}$ values. Recall that according to Theorem 5.3 we have

$$
\begin{aligned}
\tilde{s}_{k} & \leqslant S_{k}(\mathbf{N}, \mathbf{M}, \mathbf{s}) \\
& =\max \left\{\left\|P_{N_{k}}^{N_{k-1}} U \eta\right\|^{2}:\|\eta\|_{l^{\infty}} \leqslant 1,\left|\operatorname{supp}\left(P_{M_{l}}^{M_{l-1}} \eta\right)\right|=s_{l}, l=1, \ldots, r\right\},
\end{aligned}
$$

where $N_{0}=M_{0}=0$. Thus, we concentrate on bounding $S_{k}$. First note that by a direct rearrangement of terms in Lemma 7.19, for any $\gamma \in(0,1)$ and $R \in \mathbb{N}$ such that $M \leqslant W_{R}$, we have that $\left\|P_{N}^{\perp} U P_{M}\right\| \leqslant \gamma$ whenever $N$ is such that

$$
\gamma \geqslant\left(\frac{2^{R}}{\omega N}\right)^{2 \alpha-1 / 2} \cdot \sqrt{\frac{2}{2 \alpha-1}} \cdot \frac{C_{\Phi}}{\pi^{\alpha}} .
$$

So for any $L>0$, by letting $\gamma=\sqrt{2 /(2 \alpha-1)} \cdot\left(C_{\Phi} / \pi^{\alpha}\right) \cdot L^{-((2 \alpha-1) / 2)}$, if $\gamma \in(0,1)$, then $\left\|P_{N}^{\perp} U P_{M}\right\| \leqslant \gamma$ provided that $N \geqslant \omega^{-1} \cdot L \cdot 2^{R}$. Also, if $\gamma>1$, then $\left\|P_{N}^{\perp} U P_{M}\right\| \leqslant \gamma$ is trivially true since $\|U\|=1$. Therefore, for $k \geqslant 2$ we have that

$$
\left\|P_{N_{k-1}}^{\perp} U P_{M_{l}}\right\|<\sqrt{\frac{2}{2 \alpha-1}} \cdot \frac{C_{\Phi}}{\pi^{\alpha}} \cdot\left(\frac{2^{R_{l}}}{2^{R_{k-1}}}\right)^{\alpha-1 / 2}, \quad l \leqslant k-1 .
$$


Also, by Lemma 7.20, it follows that

$$
\left\|P_{N_{k}} U P_{M_{l}}^{M_{l-1}}\right\|<(2 \pi)^{v} \cdot\left\|\theta_{\Psi}\right\|_{L^{\infty}} \cdot\left(\frac{2^{R_{k}}}{2^{R_{l-1}}}\right)^{v}, \quad l \geqslant k+1 .
$$

Consequently, for $k=3, \ldots, r$

$$
\begin{aligned}
\sqrt{\tilde{s}_{k}} \leqslant & \sqrt{S_{k}}=\max _{\eta \in \Theta}\left\|P_{N_{k}}^{N_{k-1}} U \eta\right\| \leqslant \sum_{l=1}^{r}\left\|P_{N_{k}}^{N_{k-1}} U P_{M_{l}}^{M_{l-1}}\right\| \sqrt{s_{l}} \\
\leqslant & B_{\Phi, \Psi}\left(\sum_{l=1}^{k-2} \sqrt{s_{l}} \cdot\left(\frac{2^{R_{l}}}{2^{R_{k-1}}}\right)^{\alpha-1 / 2}+\sqrt{s_{k-1}}+\sqrt{s_{k}}+\sqrt{s_{k+1}}\right. \\
& \left.+\sum_{l=k+2}^{r} \sqrt{s_{l}} \cdot\left(\frac{2^{R_{k}}}{2^{R_{l-1}}}\right)^{v}\right)
\end{aligned}
$$

where

$$
\Theta=\left\{\eta:\|\eta\|_{l^{\infty}} \leqslant 1,\left|\operatorname{supp}\left(P_{M_{l}}^{M_{l-1}} \eta\right)\right|=s_{l}, l=1, \ldots, r\right\},
$$

and for $k=1,2$ we have

$$
\sqrt{\tilde{s}_{k}} \leqslant B_{\Phi, \Psi}\left(\sqrt{s_{k-1}}+\sqrt{s_{k}}+\sqrt{s_{k+1}}+\sum_{l=k+2}^{r} \sqrt{s_{l}} \cdot\left(\frac{2^{R_{k}}}{2^{R_{l-1}}}\right)^{v}\right),
$$

where we let $s_{0}=0$. Hence, for $k=3, \ldots, r, A_{\alpha}=2^{\alpha-1 / 2}$ and $A_{v}=2^{v}$

$$
\tilde{s}_{k} \leqslant B_{\Phi, \Psi}\left(\sqrt{\hat{s}_{k}}+\sum_{l=1}^{k-2} \sqrt{s_{l}} \cdot A_{\alpha}^{-\left(R_{k-1}-R_{l}\right)}+\sum_{l=k+2}^{r} \sqrt{s_{l}} \cdot A_{v}^{-\left(R_{l-1}-R_{k}\right)}\right)^{2},
$$

where $\hat{s}_{k}=\max \left\{s_{k-1}, s_{k}, s_{k+1}\right\}$. So, by using the Cauchy-Schwarz inequality, we obtain

$$
\begin{aligned}
\tilde{s}_{k} \leqslant & B_{\Phi, \Psi}\left(1+\sum_{l=1}^{k-2} A_{\alpha}^{-\left(R_{k-1}-R_{l}\right)}+\sum_{l=k+2}^{r} A_{v}^{-\left(R_{l-1}-R_{k}\right)}\right) \\
& \times\left(\hat{s}_{k}+\sum_{l=1}^{k-2} s_{l} \cdot A_{\alpha}^{-\left(R_{k-1}-R_{l}\right)}+\sum_{l=k+2}^{r} s_{l} \cdot A_{v}^{-\left(R_{l-1}-R_{k}\right)}\right) \\
\leqslant & B_{\Phi, \Psi}\left(\hat{s}_{k}+\sum_{l=1}^{k-2} s_{l} \cdot A_{\alpha}^{-\left(R_{k-1}-R_{l}\right)}+\sum_{l=k+2}^{r} s_{l} \cdot A_{v}^{-\left(R_{l-1}-R_{k}\right)}\right),
\end{aligned}
$$

and similarly, for $k=1,2$, it follows that $\tilde{s}_{k} \leqslant B_{\Phi, \Psi}\left(\hat{s}_{k}+\sum_{l=k+2}^{r} s_{l} \cdot A_{v}^{-\left(R_{l-1}-R_{k}\right)}\right)$. Finally, we use the above results to show that condition (6.1) implies (7.121): 
By our coherence estimates in (7.110), (7.112), (7.111) and (7.113), we see that (7.121) holds if $m_{k} \gtrsim \hat{m}_{k} \cdot\left(\log \left(\epsilon^{-1}\right)+1\right) \cdot \log \left((K \sqrt{s})^{1+1 / v} N\right)$ and for each $l=2$, $\ldots, r$,

$$
\begin{aligned}
1 \gtrsim & B_{\Phi, \Psi}\left(\left(\frac{N_{1}}{\hat{m}_{1}}-1\right) \cdot \tilde{s}_{1} \cdot \sqrt{\frac{\omega}{2^{R_{l-1}}}} \cdot\left(\frac{\omega N_{1}}{2^{R_{l-1}}}\right)^{v}\right. \\
& +\sum_{k=2}^{l-1}\left(\frac{N_{k}-N_{k-1}}{\hat{m}_{k}}-1\right) \cdot \tilde{s}_{k} \cdot \sqrt{\frac{\omega}{N_{k-1} 2^{R_{l-1}}}} \cdot\left(\frac{\omega N_{k}}{2^{R_{l-1}}}\right)^{v} \\
& +\left(\frac{N_{l}-N_{l-1}}{\hat{m}_{l}}-1\right) \cdot \tilde{s}_{l} \cdot \frac{1}{N_{l-1}} \\
& \left.+\sum_{k=l+1}^{r}\left(\frac{N_{k}-N_{k-1}}{\hat{m}_{k}}-1\right) \cdot \tilde{s}_{k} \cdot \frac{1}{N_{k-1}}\left(\frac{2^{R_{l-1}}}{\omega N_{k-1}}\right)^{\alpha-1 / 2}\right),
\end{aligned}
$$

(where we with slight abuse of notation define

$$
\sum_{k=2}^{l-1}\left(\frac{N_{k}-N_{k-1}}{\hat{m}_{k}}-1\right) \tilde{s}_{k} \sqrt{\frac{\omega}{N_{k-1} 2^{R_{l-1}}}}\left(\frac{\omega N_{k}}{2^{R_{l-1}}}\right)^{v}=0
$$

when $l=2)$, and for $l=1$

$$
\begin{aligned}
1 \gtrsim B_{\Phi, \Psi} & \left(\left(\frac{N_{1}}{\hat{m}_{1}}-1\right) \cdot \tilde{s}_{1}\right. \\
& \left.+\sum_{k=2}^{r}\left(\frac{N_{k}-N_{k-1}}{\hat{m}_{k}}-1\right) \cdot \tilde{s}_{k} \cdot \frac{1}{N_{k-1}}\left(\frac{1}{\omega N_{k-1}}\right)^{\alpha-1 / 2}\right) .
\end{aligned}
$$

Recalling that $N_{k}=\omega^{-1} 2^{R_{k}},(7.127)$ becomes, for $l=2, \ldots, r$,

$$
\begin{aligned}
1 \gtrsim & B_{\Phi, \Psi} \cdot\left(\left(\frac{N_{1}}{\hat{m}_{1}}-1\right) \cdot \frac{\tilde{s}_{k}}{N_{k-1}} \cdot 2^{-v\left(R_{l-1}-R_{k}\right)}\right. \\
& +\sum_{k=1}^{l-1}\left(\frac{N_{k}-N_{k-1}}{\hat{m}_{k}}-1\right) \cdot \frac{\tilde{s}_{k}}{N_{k-1}} \cdot 2^{-v\left(R_{l-1}-R_{k}\right)}+\left(\frac{N_{l}-N_{l-1}}{\hat{m}_{l}}-1\right) \cdot \frac{\tilde{s}_{l}}{N_{l-1}} \\
& \left.+\sum_{k=l+1}^{r}\left(\frac{N_{k}-N_{k-1}}{\hat{m}_{k}}-1\right) \cdot \frac{\tilde{s}_{k}}{N_{k-1}} \cdot\left(2^{\alpha-1 / 2}\right)^{-\left(R_{k-1}-R_{l-1}\right)}\right),
\end{aligned}
$$

and (7.128) becomes

$$
1 \gtrsim B_{\Phi, \Psi} \cdot\left(\left(\frac{N_{1}}{\hat{m}_{1}}-1\right) \cdot \tilde{s}_{1}+\sum_{k=l+1}^{r}\left(\frac{N_{k}-N_{k-1}}{\hat{m}_{k}}-1\right) \cdot \frac{\tilde{s}_{k}}{N_{k-1}} \cdot\left(2^{\alpha-1 / 2}\right)^{-R_{k-1}}\right) .
$$


Observe that for $l=2, \ldots, r$

$$
1+\sum_{k=1}^{l-1} 2^{-v\left(R_{l-1}-R_{k}\right)}+\sum_{k=l+1}^{r}\left(2^{\alpha-1 / 2}\right)^{-\left(R_{k-1}-R_{l-1}\right)} \leqslant B_{\Phi, \Psi},
$$

and that $1+\sum_{k=l+1}^{r}\left(2^{\alpha-1 / 2}\right)^{-\left(R_{k-1}\right)} \leqslant B_{\Phi, \Psi}$. Thus, (7.121) holds provided that for each $k=2, \ldots, r$,

$$
\hat{m}_{k} \geqslant B_{\Phi, \Psi} \cdot \frac{N_{k}-N_{k-1}}{N_{k-1}} \cdot \tilde{s}_{k}, \quad \hat{m}_{1} \geqslant B_{\Phi, \Psi} \cdot N_{1} \cdot \tilde{s}_{1},
$$

and combining with our estimates of $\tilde{s}_{k}$, we may deduce that (6.1) implies (7.121).

Proof of Proposition 6.4. If $\left\|P_{J}^{\perp} x\right\|=0$, there is nothing to prove, thus, we assume that $\left\|P_{J}^{\perp} x\right\| \neq 0$. Let $f=\sum_{j=1}^{\infty} x_{j} \varphi_{j}$ and $\hat{f}=\left\{\hat{f}_{j}\right\}_{j \in \mathbb{N}}$, where $\hat{f}_{j}=$ $\left\langle f, \psi_{j}\right\rangle$. Similarly, let $g=\sum_{j=1}^{J} x_{j} \varphi_{j}$ and $\hat{g}=\left\{\hat{g}_{j}\right\}_{j \in \mathbb{N}}$, where $\hat{g}_{j}=\left\langle g, \psi_{j}\right\rangle$. Let $\Omega$ be a multilevel sampling scheme as in Theorem 6.2, and define $y=$ $P_{\Omega} \hat{f}+z$ where $z \in \operatorname{ran}\left(P_{\Omega}\right)$ is a noise vector satisfying $\|z\| \leqslant \delta$. Now, let $z_{1}=P_{\Omega} U P_{J} x-y$. Suppose for the moment that $\left\|P_{\Omega} U P_{J}^{\perp}\right\|\left\|P_{J}^{\perp} x\right\| \leqslant \delta$, we show this later. Then we have

$$
\left\|z_{1}\right\| \leqslant\left\|P_{\Omega} U x-y\right\|+\left\|P_{\Omega} U P_{J}^{\perp} x\right\| \leqslant \delta+\left\|P_{\Omega} U P_{J}^{\perp}\right\|\left\|P_{J}^{\perp} x\right\| \leqslant 2 \delta .
$$

Define $\tilde{y}=P_{\Omega} \hat{g}-z_{1}$ and apply Theorem 6.2 to $g$ and the noise vector $z_{1}$. Then, since $\tilde{y}=y$ we get that any minimizer $\tilde{\xi}$ of

$$
\min _{\eta \in \mathbb{C}^{J}}\|\eta\|_{l^{1}} \text { subject to }\left\|P_{\Omega} U P_{J} \eta-y\right\| \leqslant 2 \delta,
$$

satisfies

$$
\left\|\tilde{\xi}-P_{J} x\right\| \leqslant C \cdot\left(2 \tilde{\delta} \cdot(1+L \cdot \sqrt{s})+\sigma_{\mathbf{s}, \mathbf{M}}(g)\right) .
$$

However, $\sigma_{\mathbf{s}, \mathbf{M}}(g) \leqslant \sigma_{\mathbf{s}, \mathbf{M}}(f)$ and $\left\|P_{J}^{\perp} x\right\| \leqslant \sigma_{\mathbf{s}, \mathbf{M}}(f)$. Thus,

$$
\|\tilde{\xi}-x\| \leqslant 2 C \cdot\left(\tilde{\delta} \cdot(1+L \cdot \sqrt{s})+\sigma_{\mathbf{s}, \mathbf{M}}(f)\right),
$$

where we have assumed without loss of generality that $C \geqslant 1$. So, to finish the proof, we only need to show that $\left\|P_{\Omega} U P_{J}^{\perp}\right\|\left\|P_{J}^{\perp} x\right\| \leqslant \delta$. In fact, we show that $\left\|P_{N} U P_{J}^{\perp}\right\|\left\|P_{J}^{\perp} x\right\| \leqslant \delta$. To see the latter, by choosing $R_{1}=R$ and letting $R_{2} \rightarrow \infty$ in Lemma 7.20, it follows that for any $\gamma \in(0,1)$

$$
\left\|P_{N} U P_{J}^{\perp}\right\| \leqslant \frac{\pi^{2}}{4}\left\|\theta_{\Psi}\right\|_{L^{\infty}}(2 \pi \gamma)^{v} \sqrt{2}
$$


whenever $N \leqslant \gamma 2^{R} \omega^{-1}$. Letting

$$
\gamma=(2 \pi)^{-1}\left(\frac{\delta}{\left\|P_{J}^{\perp} x\right\| \cdot\left\|\theta_{\Psi}\right\|_{L^{\infty}} \pi^{2}}\right)^{1 / v}
$$

we get the desired bound when $2^{R} \geqslant 2 \pi \omega N \cdot\left(\left(\left(\left\|P_{K}^{\perp} x\right\|_{2} \cdot C_{\Psi}\right) / \delta\right)\right)^{1 / v}$, where $C_{\Psi}^{-1}=\left\|\theta_{\Psi}\right\|_{L^{\infty}} \pi^{2}$.

\section{Acknowledgements}

The authors would like to thank Akram Aldroubi, Emmanuel Candès, Massimo Fornasier, Karlheinz Gröchenig, Felix Krahmer, Gitta Kutyniok, Thomas Strohmer, Gerd Teschke, Michael Unser, Martin Vetterli and Rachel Ward for useful discussions and comments. The authors also thank Stuart Marcelle and Homerton College, University of Cambridge for the provision of computing hardware used in some of the experiments. B.A. acknowledges support from the NSF DMS grant 1318894. A.C.H. acknowledges support from a Royal Society University Research Fellowship as well as the UK Engineering and Physical Sciences Research Council (EPSRC) grant EP/L003457/1. C.P. acknowledges support from the EPSRC grant EP/H023348/1 for the University of Cambridge Centre for Doctoral Training, the Cambridge Centre for Analysis.

\section{References}

[1] B. Adcock and A. C. Hansen, 'A generalized sampling theorem for stable reconstructions in arbitrary bases', J. Fourier Anal. Appl. 18(4) (2012), 685-716.

[2] B. Adcock and A. C. Hansen, 'Stable reconstructions in Hilbert spaces and the resolution of the Gibbs phenomenon', Appl. Comput. Harmon. Anal. 32(3) (2012), 357-388.

[3] B. Adcock and A. C. Hansen, 'Generalized sampling and infinite-dimensional compressed sensing', Found. Comput. Math. 16(5) (2016), 1263-1323.

[4] B. Adcock, A. C. Hansen, E. Herrholz and G. Teschke, 'Generalized sampling: extension to frames and inverse and ill-posed problems', Inverse Problems 29(1) (2013), 015008.

[5] B. Adcock, A. C. Hansen and C. Poon, 'Beyond consistent reconstructions: optimality and sharp bounds for generalized sampling, and application to the uniform resampling problem', SIAM J. Math. Anal. 45(5) (2013), 3114-3131.

[6] B. Adcock, A. C. Hansen and C. Poon, 'On optimal wavelet reconstructions from Fourier samples: linearity and universality of the stable sampling rate', Appl. Comput. Harmon. Anal. 36(3) (2014), 387-415.

[7] B. Adcock, A. C. Hansen, B. Roman and G. Teschke, 'Generalized sampling: stable reconstructions, inverse problems and compressed sensing over the continuum', Adv. Imaging Electron Phys. 182 (2014), 187-279.

[8] R. G. Baraniuk, V. Cevher, M. F. Duarte and C. Hedge, 'Model-based compressive sensing', IEEE Trans. Inform. Theory 56(4) (2010), 1982-2001. 
[9] A. Bastounis and A. C. Hansen, 'On the absence of uniform recovery in many real-world applications of compressed sensing and the RIP \& nullspace property in levels', SIAM J. Imaging Sci. (to appear).

[10] J. Bigot, C. Boyer and P. Weiss, 'An analysis of block sampling strategies in compressed sensing', IEEE Trans. Inform. Theory 62(4) (2016), 2125-2139.

[11] A. Bourrier, M. E. Davies, T. Peleg, P. Pérez and R. Gribonval, 'Fundamental performance limits for ideal decoders in high-dimensional linear inverse problems', IEEE Trans. Inform. Theory 60(12) (2014), 7928-7946.

[12] C. Boyer, J. Bigot and P. Weiss, 'Compressed sensing with structured sparsity and structured acquisition', Preprint, 2015, arXiv:1505.01619.

[13] E. Candès and D. L. Donoho, 'Recovering edges in ill-posed inverse problems: optimality of curvelet frames', Ann. Statist. 30(3) (2002), 784-842.

[14] E. J. Candès, 'An introduction to compressive sensing', IEEE Signal Process. Mag. 25(2) (2008), 21-30.

[15] E. J. Candès and D. Donoho, 'New tight frames of curvelets and optimal representations of objects with piecewise $C^{2}$ singularities', Comm. Pure Appl. Math. 57(2) (2004), 219-266.

[16] E. J. Candès and Y. Plan, 'A probabilistic and RIPless theory of compressed sensing', IEEE Trans. Inform. Theory 57(11) (2011), 7235-7254.

[17] E. J. Candès and J. Romberg, 'Sparsity and incoherence in compressive sampling', Inverse Problems 23(3) (2007), 969-985.

[18] E. J. Candès, J. Romberg and T. Tao, 'Robust uncertainty principles: exact signal reconstruction from highly incomplete frequency information', IEEE Trans. Inform. Theory 52(2) (2006), 489-509.

[19] W. R. Carson, M. Chen, M. R. D. Rodrigues, R. Calderbank and L. Carin, 'Communicationsinspired projection design with application to compressive sensing', SIAM J. Imaging Sci. 5(4) (2012), 1185-1212.

[20] N. Chauffert, P. Ciuciu, J. Kahn and P. Weiss, 'Variable density sampling with continuous trajectories', SIAM J. Imaging Sci. 7(4) (2014), 1962-1992.

[21] N. Chauffert, P. Weiss, J. Kahn and P. Ciuciu, 'Gradient waveform design for variable density sampling in magnetic resonance imaging', Preprint, 2014, arXiv:1412.4621.

[22] Y. Chi, L. L. Scharf, A. Pezeshki and R. Calderbank, 'Sensitivity to basis mismatch in compressed sensing', IEEE Trans. Signal Process. 59(5) (2011), 2182-2195.

[23] A. Cohen, W. Dahmen and R. DeVore, 'Compressed sensing and best $k$-term approximation', J. Amer. Math. Soc. 22(1) (2009), 211-231.

[24] T. H. Cormen, C. Stein, R. L. Rivest and C. E. Leiserson, Introduction to Algorithms, 2nd edn (MIT Press, Cambridge, MA; McGraw-Hill Book Co., Boston, MA, 2001).

[25] S. Dahlke, G. Kutyniok, P. Maass, C. Sagiv, H.-G. Stark and G. Teschke, 'The uncertainty principle associated with the continuous shearlet transform', Int. J. Wavelets Multiresolut. Inf. Process. 6(2) (2008), 157-181.

[26] S. Dahlke, G. Kutyniok, G. Steidl and G. Teschke, 'Shearlet coorbit spaces and associated Banach frames', Appl. Comput. Harmon. Anal. 27(2) (2009), 195-214.

[27] I. Daubechies, 'Orthonormal bases of compactly supported wavelets', Comm. Pure Appl. Math. 41(7) (1988), 909-996.

[28] I. Daubechies, Ten Lectures on Wavelets, CBMS-NSF Regional Conference Series in Applied Mathematics (Society for Industrial and Applied Mathematics (SIAM), Philadelphia, PA, 1992).

[29] M. A. Davenport, M. F. Duarte, Y. C. Eldar and G. Kutyniok, 'Introduction to compressed sensing', in Compressed Sensing: Theory and Applications (Cambridge University Press, Cambridge, 2011). 
[30] R. A. DeVore, 'Nonlinear approximation', Acta Numer. 7 (1998), 51-150.

[31] M. N. Do and M. Vetterli, 'The contourlet transform: an efficient directional multiresolution image representation', IEEE Trans. Image Process. 14(12) (2005), 2091-2106.

[32] D. L. Donoho, 'Compressed sensing', IEEE Trans. Inform. Theory 52(4) (2006), 1289-1306.

[33] D. L. Donoho and X. Huo, 'Uncertainty principles and ideal atomic decomposition', IEEE Trans. Inform. Theory 47 (2001), 2845-2862.

[34] D. L. Donoho and G. Kutyniok, 'Microlocal analysis of the geometric separation problem', Comm. Pure Appl. Math. 66(1) (2013), 1-47.

[35] D. L. Donoho and J. Tanner, 'Neighborliness of randomly-projected simplices in high dimensions', Proc. Natl Acad. Sci. USA 102(27) (2005), 9452-9457.

[36] D. L. Donoho and J. Tanner, 'Counting faces of randomly-projected polytopes when the projection radically lowers dimension', J. Amer. Math. Soc. 22(1) (2009), 1-53.

[37] M. F. Duarte, M. A. Davenport, D. Takhar, J. Laska, K. Kelly and R. G. Baraniuk, 'Singlepixel imaging via compressive sampling', IEEE Signal Process. Mag. 25(2) (2008), 83-91.

[38] M. F. Duarte and Y. C. Eldar, 'Structured compressed sensing: from theory to applications', IEEE Trans. Signal Process. 59(9) (2011), 4053-4085.

[39] Y. C. Eldar and G. Kutyniok (Eds.), Compressed Sensing: Theory and Applications (Cambridge University Press, Cambridge, 2012).

[40] M. Fornasier and H. Rauhut, 'Compressive sensing', in Handbook of Mathematical Methods in Imaging (Springer, New York, NY, 2011), 187-228.

[41] S. Foucart and H. Rauhut, A Mathematical Introduction to Compressive Sensing (Birkhäuser/Springer, New York, NY, 2013).

[42] K. Gröchenig, Z. Rzeszotnik and T. Strohmer, 'Convergence analysis of the finite section method and banach algebras of matrices', Integr. Equat. Oper. Th. 67(2) (2010), 183-202.

[43] D. Gross, 'Recovering low-rank matrices from few coefficients in any basis', IEEE Trans. Inform. Theory 57(3) (2011), 1548-1566.

[44] D. Gross, F. Krahmer and R. Kueng, 'A partial derandomization of phaselift using spherical designs', J. Fourier Anal. Appl. 21(2) (2015), 229-266.

[45] M. Guerquin-Kern, M. Häberlin, K. Pruessmann and M. Unser, 'A fast wavelet-based reconstruction method for magnetic resonance imaging', IEEE Transactions on Medical Imaging 30(9) (2011), 1649-1660.

[46] M. Guerquin-Kern, L. Lejeune, K. P. Pruessmann and M. Unser, 'Realistic analytical phantoms for parallel Magnetic Resonance Imaging', IEEE Trans. Med. Imaging 31(3) (2012), 626-636.

[47] A. C. Hansen, 'On the approximation of spectra of linear operators on hilbert spaces', J. Funct. Anal. 254(8) (2008), 2092-2126.

[48] A. C. Hansen, 'On the solvability complexity index, the $n$-pseudospectrum and approximations of spectra of operators', J. Amer. Math. Soc. 24(1) (2011), 81-124.

[49] M. A. Herman, 'Compressive sensing with partial-complete, multiscale Hadamard waveforms', in Imaging and Applied Optics (Optical Society of America, Arlington, VA, 2013), CM4C.3.

[50] M. A. Herman, T. Weston, L. McMackin, Y. Li, J. Chen and K. F. Kelly, 'Recent results in single-pixel compressive imaging using selective measurement strategies', in Proc. SPIE 9484, Compressive Sensing IV 94840A (SPIE, Baltimore, MD, 2015).

[51] E. Hernández and G. Weiss, A First Course on Wavelets, Studies in Advanced Mathematics (CRC Press, Boca Raton, FL, 1996). 
[52] T. Hrycak and K. Gröchenig, 'Pseudospectral Fourier reconstruction with the modified inverse polynomial reconstruction method', J. Comput. Phys. 229(3) (2010), 933-946.

[53] A. Jones, A. Tamtögl, I. Calvo-Almazán and A. Hansen, 'Continuous compressed sensing for surface dynamical processes with helium atom scattering', Sci. Rep. 6 (2016), 27776 $\mathrm{EP}-, 06$.

[54] A. D. Jones, B. Adcock and A. C. Hansen, 'On asymptotic incoherence and its implications for compressed sensing of inverse problems', IEEE Trans. Inform. Theory 62(2) (2016), 1020-1037.

[55] F. Krahmer and R. Ward, 'Stable and robust sampling strategies for compressive imaging', IEEE Trans. Image Process. 23(2) (2014), 612-622.

[56] G. Kutyniok, J. Lemvig and W.-Q. Lim, 'Compactly supported shearlets', in Approximation Theory XIII: San Antonio 2010, (eds. M. Neamtu and L. Schumaker) Springer Proceedings in Mathematics, 13 (Springer, New York, 2012), 163-186.

[57] G. Kutyniok and W.-Q. Lim, 'Optimal compressive imaging of Fourier data', Preprint, 2015, arXiv:1510.05029.

[58] P. E. Z. Larson, S. Hu, M. Lustig, A. B. Kerr, S. J. Nelson, J. Kurhanewicz, J. M. Pauly and D. B. Vigneron, 'Fast dynamic 3D MR spectroscopic imaging with compressed sensing and multiband excitation pulses for hyperpolarized 13c studies', Magn. Reson. Med. 65(3) (2011), 610-619.

[59] M. Ledoux, The Concentration of Measure Phenomenon, Mathematical Surveys and Monographs, 89 (American Mathematical Society, Providence, RI, 2001).

[60] C. Li and B. Adcock, 'Compressed sensing with local structure: uniform recovery guarantees for the sparsity in levels class', Preprint, 2016, arXiv:1601.01988.

[61] M. Lustig, 'Sparse MRI', PhD Thesis, Stanford University, 2008.

[62] M. Lustig, D. L. Donoho and J. M. Pauly, 'Sparse MRI: the application of compressed sensing for rapid MRI imaging', Magn. Reson. Imaging 58(6) (2007), 1182-1195.

[63] M. Lustig, D. L. Donoho, J. M. Santos and J. M. Pauly, 'Compressed Sensing MRI', IEEE Signal Process. Mag. 25(2) (2008), 72-82.

[64] S. G. Mallat, A Wavelet Tour of Signal Processing: The Sparse Way, 3rd edn (Elsevier/ Academic Press, Amsterdam, 2009).

[65] C. McDiarmid, 'Concentration', in Probabilistic Methods for Algorithmic Discrete Mathematics, Algorithms and Combinatorics, 16 (Springer, Berlin, 1998), 195-248.

[66] D. D.-Y. Po and M. N. Do, 'Directional multiscale modeling of images using the contourlet transform', IEEE Trans. Image Process. 15(6) (2006), 1610-1620.

[67] C. Poon, 'A consistent, and stable approach to generalized sampling', J. Fourier Anal. Appl. 20(5) (2014), 985-1019.

[68] C. Poon, 'On the role of total variation in compressed sensing', SIAM J. Imaging Sci. 8(1) (2015), 682-720.

[69] C. Poon, 'Structure dependent sampling in compressed sensing: theoretical guarantees for tight frames', Appl. Comput. Harmon. Anal. (2015), (to appear).

[70] G. Puy, J. P. Marques, R. Gruetter, J. Thiran, D. Van De Ville, P. Vandergheynst and Y. Wiaux, 'Spread spectrum Magnetic Resonance Imaging', IEEE Trans. Med. Imaging 31(3) (2012), 586-598.

[71] G. Puy, P. Vandergheynst and Y. Wiaux, 'On variable density compressive sampling', IEEE Signal Process. Letters 18 (2011), 595-598. 
[72] H. Rauhut and R. Ward, 'Interpolation via weighted $\ell^{1}$ minimization', Appl. Comput. Harmon. Anal. 40(2) (2016), 321-351.

[73] B. Roman, B. Adcock and A. C. Hansen, 'On asymptotic structure in compressed sensing', Preprint, 2014, arXiv:1406.4178.

[74] J. Romberg, 'Imaging via compressive sampling', IEEE Signal Process. Mag. 25(2) (2008), 14-20.

[75] M. Rudelson, 'Random vectors in the isotropic position', J. Funct. Anal. 164(1) (1999), 60-72.

[76] T. Strohmer, 'Measure what should be measured: progress and challenges in compressive sensing', IEEE Signal Process. Letters 19(12) (2012), 887-893.

[77] V. Studer, J. Bobin, M. Chahid, H. S. Mousavi, E. Candès and M. Dahan, 'Compressive fluorescence microscopy for biological and hyperspectral imaging', Proc. Natl. Acad. Sci. USA 109(26) (2012), E1679-E1687.

[78] D. Takhar, J. N. Laska, M. B. Wakin, M. F. Duarte, D. Baron, S. Sarvotham, K. F. Kelly and R. G. Baraniuk, 'A new compressive imaging camera architecture using optical-domain compression', in Proc. of Computational Imaging IV at SPIE Electronic Imaging (2006), 43-52.

[79] M. Talagrand, 'New concentration inequalities in product spaces', Invent. Math. 126(3) (1996), 505-563.

[80] Y. Traonmilin and R. Gribonval, 'Stable recovery of low-dimensional cones in Hilbert spaces: one RIP to rule them all', Appl. Comput. Harmon. Anal. (2016), (to appear).

[81] J. A. Tropp, 'On the conditioning of random subdictionaries', Appl. Comput. Harmon. Anal. 25(1) (2008), 1-24.

[82] Y. Tsaig and D. L. Donoho, 'Extensions of compressed sensing', Signal Process. 86(3) (2006), 549-571.

[83] L. Wang, D. Carlson, M. R. D. Rodrigues, D. Wilcox, R. Calderbank and L. Carin, 'Designed measurements for vector count data', in Advances in Neural Information Processing Systems (2013), 1142-1150.

[84] Q. Wang, M. Zenge, H. E. Cetingul, E. Mueller and M. S. Nadar, 'Novel sampling strategies for sparse mr image reconstruction', Proc. Int. Soc. Mag. Res. in Med. (22) (2014).

[85] Z. Wang and G. R. Arce, 'Variable density compressed image sampling', IEEE Trans. Image Process. 19(1) (2010), 264-270.

[86] A. Zomet and S. K. Nayar, 'Lensless imaging with a controllable aperture', in 2006 IEEE Computer Society Conference on Computer Vision and Pattern Recognition (IEEE, 2006), 339-346. 